\title{
Review and Comparison of Equations of State for the Lennard-Jones Fluid
}

\author{
Simon Stephan, ${ }^{1, \text { a) }}$ Jens Staubach, ${ }^{1}$ and Hans Hasse ${ }^{1}$ \\ Laboratory of Engineering Thermodynamics (LTD), TU Kaiserslautern, \\ Kaiserslautern, Germany
}

(Dated: Tuesday $11^{\text {th }}$ August, 2020)

The Lennard-Jones (LJ) potential is widely used for describing simple fluids; it is also a point of departure for developing models of complex fluids. Thermodynamic properties of the LJ fluid have been studied by molecular simulations by many authors and a critical review of the available data, which comprises about 35,000 data points, has been published recently [J. Chem. Inf. Mod. 59 (2019) 4248-4265]. The importance of the LJ fluid has also triggered the development of a large number of equations of state (EOS). In the present work, 20 LJ EOS were critically assessed by comparing their results with consolidated data from computer experiments. A large variety of thermophysical properties was considered: vapor pressure; saturated densities; enthalpy of vaporization; critical properties; thermal, caloric, and entropic properties at homogeneous state points; and second and third virial coefficients. It was found that none of the available LJ EOS meets the following two criteria: (1) it does not yield unphysical artifacts when used for extrapolations, and (2) it describes data from computer experiments within their statistical uncertainty in most fluid regions. Furthermore, a re-parameterization of the monomer term of the PC-SAFT EOS was carried out by fitting it to data of the LJ fluid. The new LJ EOS yields good results for the LJ fluid, but does not outperform the best existing LJ EOS.

Keywords: Lennard-Jones fluid, equation of state, molecular simulation, monomer reference fluid, SAFT

a) Electronic mail: simon.stephan@mv.uni-kl.de 


\section{INTRODUCTION}

The Lennard-Jones potential ${ }^{1,2}$ has been used extensively as a model of simple fluids with repulsive and dispersive interactions since the early days of computer simulation ${ }^{3-10}$. It is defined as

$$
u_{\mathrm{LJ}}(r)=4 \varepsilon\left[\left(\frac{\sigma}{r}\right)^{12}-\left(\frac{\sigma}{r}\right)^{6}\right],
$$

where $\varepsilon$ and $\sigma$ are the energy and size parameter, respectively. The distance between two particles is denoted by $r$. It is probably the most frequently investigated monomer model fluid ${ }^{11}$. The Lennard-Jones potential provides, as already stated by Nicolas et al. in $1979^{12}$, "a convenient model for testing liquid theories and for investigating such phenomena as melting, the liquid-vapor surface, nucleation etc.". This has not changed in the past

four decades ${ }^{13-24}$. Furthermore, the Lennard-Jones potential is used as a building block in molecular models of more complex fluids ${ }^{25-27}$. The Lennard-Jones potential is referred to as LJ potential in the following.

A large number of equations of state (EOS) of the LJ fluid have been proposed in the literature of which Table I gives a survey. To the best of our knowledge, the different LJ EOS from the literature have never been compared systematically. We have therefore compared 20 of the most widely used LJ EOS using a consistent approach and consolidated reference data. The LJ EOS that were included in the present study are marked with abbreviations in Table I. The focus of this work is on the LJ EOS developed in the past 30 years. However, some older yet still frequently used LJ EOS were also included in the comparison ${ }^{12,28-30}$.

A comprehensive database of thermophysical properties of the LJ fluid determined by computer experiments has recently become available ${ }^{11}$. There are about 35,000 data points on thermophysical properties of the LJ fluid in the literature, which were critically assessed and screened for outliers in Ref. ${ }^{11}$. The consolidated database from Ref. ${ }^{11}$ still contains about 32,000 entries and was used here as basis for the assessment of the LJ EOS.

The following physical properties were considered in the present work:

- vapor-liquid equilibrium (for given $T$ : vapor pressure $p^{\mathrm{s}}$, saturated liquid and vapor densities $\rho^{\prime}$ and $\rho^{\prime \prime}$, and enthalpy of vaporization $\left.\Delta h_{\mathrm{v}}\right)$.

- the critical point (critical temperature $T_{\mathrm{c}}$, critical density $\rho_{\mathrm{c}}$, and critical pressure $p_{\mathrm{c}}$ ).

- homogeneous states (for given temperature $T$ and density $\rho$ : pressure $p$, thermal 
expansion coefficient $\alpha$, isothermal compressibility $\beta$, thermal pressure coefficient $\gamma$, internal energy $u$, isochoric heat capacity $c_{v}$, isobaric heat capacity $c_{p}$, Grüneisen parameter $\Gamma$, Joule-Thomson coefficient $\mu_{\mathrm{JT}}$, speed of sound $w$, Helmholtz energy $a$, and chemical potential $\mu$ ) in the different fluid regions such as: gas, liquid, supercritical, etc. (details are given below). Also, second and third thermal virial coefficients $B$ and $C$ were considered.

- the qualitative behavior of $p(\rho)$ isotherms in the metastable and unstable region and the spinodal curve.

LJ EOS can be broadly classified into empirical EOS and theoretically-based EOS. This is only a crude classification and used here in the following sense ${ }^{31}$ : empirical EOS are data-driven and aim at correlating the data well. This is usually accomplished by a flexible mathematical form with a large number of parameters. In contrast, the mathematical form of theoretically-based EOS is derived from theoretical considerations, but usually also use empirical correction terms and simulation data for determining parameters. Theoreticallybased EOS usually have a smaller number of adjustable parameters than the empirical EOS. We furthermore use the class semi-theoretical for a more distinct differentiation, which comprises EOS that can not be clearly assigned to either of the above two classes. EOS from the semi-theoretical class typically incorporate dominating empirical correction terms, but also contain significant theoretically derived features. Obviously, the border between these three types of EOS is blurred and not unambiguous. In general, most LJ EOS have some physically motivated features and some empirical features, see Deiters and de Reuck ${ }^{31}$ for a detailed discussion.

It is widely accepted that both types of EOS have strengths and weaknesses that are often characterized as follows $^{31-33}$ : empirical EOS are strong regarding the accurate description of available data, but weak regarding extrapolations into regions where no data was used for the training of the EOS; for theoretically-based EOS it is the other way around: they are expected to be strong regarding extrapolations, but typically less accurate in the description of existing data. Furthermore, empirical EOS are prone to yield unphysical behavior such as crossing isotherms ${ }^{34-36}$ or several van der Waals loops in the vapor-liquid coexistence region $^{32,37,38}$.

Lennard-Jones equations of state are often used as a base model for building models of 
more complex fluids ${ }^{29,39-42}$. Among the theoretically-based EOS, those of the SAFT-type are particularly successful and have been widely used for describing complex fluids ${ }^{39,42-46}$. Most of them were developed starting from an LJ EOS for describing the monomer unit ${ }^{40,41,45,47-54}$. Different LJ EOS were used in different SAFT EOS. The monomer equations of the following SAFT EOS were included in the present study: the Lennard-Jones extended SAFT ${ }^{51,52}$, LJ$\mathrm{SAFT}^{48,49}$, LJ-based SAFT ${ }^{54}$, soft-SAFT ${ }^{40,41}$, and SAFT-VR Mie ${ }^{42,55}$; the corresponding LJ EOS considered in the present work are those from Refs. ${ }^{12,42,56,57}$. Another important SAFT EOS is PC-SAFT ${ }^{58,59}$. The monomer term of PC-SAFT was not developed to give a good description of the LJ fluid. In the present work, the monomer term of PC-SAFT was reparametrized in order to get good results for the LJ fluid and enable direct comparisons with the monomer terms in other versions of SAFT EOS. The parametrization, which aims at a good description of both the vapor-liquid equilibrium and the homogeneous region, uses elements from multi-criteria optimization. It is briefly described and summarized in the Appendix; details are given in the Supplementary Material. The results that were obtained with the new LJ EOS give insights in strengths and weaknesses of the functional form of the monomer term that is used in PC-SAFT, which is put into relation to the results from other LJ EOS.

The outline of this work is as follows: first, an overview of the LJ EOS from the literature that were considered in this work is given. The second and main part comprises the evaluation and comparison of LJ EOS. Finally, conclusions are drawn.

Throughout the present work, all physical properties are conveniently reduced using the Lennard-Jones potential parameters $\varepsilon, \sigma$ and the molecular mass $M$, as well as the Boltzmann constant $k_{\mathrm{B}}$ as described in Ref. ${ }^{60}$. The present work is limited to the fluid regions of the Lennard-Jones fluid, i.e. no solid phases are considered. This includes the two-phase regions in which solids occur. The term two-phase region refers therefore exclusively to the vapor-liquid coexistence region here.

\section{OVERVIEW OF LENNARD-JONES EOS}

Table I gives an overview of LJ EOS from the literature. Equations of state that are only defined piecewise are not considered in Table I, since their derivatives can be discontinuous. 
TABLE I. Overview of Lennard-Jones equations of state from the literature - sorted chronologically. \# indicates the number of parameters of the LJ EOS. LJ EOS indicated with an abbreviation were included in the comparison in the present work.

\begin{tabular}{|c|c|c|c|c|}
\hline Authors & Abbr. & EOS-type & Year & $\#$ \\
\hline Levesque and Verlet $t^{61}$ & & $\mathrm{BH}$ perturbation theory; pressure explicit & 1969 & 9 \\
\hline Toxvaerd and Praestgaard ${ }^{62}$ & & $\mathrm{BH}$ perturbation theory; pressure explicit & 1970 & 8 \\
\hline McDonald and Singer ${ }^{63}$ & & empirical; Helmholtz energy explicit & 1972 & 20 \\
\hline Nicolas et al. ${ }^{12}$ & $\mathrm{Ni}$ & empirical (MBWR); pressure explicit & 1979 & 33 \\
\hline $\operatorname{Re}^{28}$ & $\operatorname{Re}$ & empirical; pressure explicit & 1980 & 15 \\
\hline Sys and Malijevski ${ }^{64}$ & & empirical + virial; pressure explicit & 1980 & 21 \\
\hline Cotterman et al. ${ }^{29}$ & Co & BH perturbation theory + virial; Helmholtz energy explicit & 1986 & 15 \\
\hline Adachi et al. ${ }^{30}$ & Ad & empirical (MBWR); pressure explicit & 1988 & 33 \\
\hline Song and Mason ${ }^{65}$ & & modified van der Waals equation + virial; pressure explicit & 1989 & 0 \\
\hline Nezbeda and Aim $^{66}$ & & empirical; pressure explicit & 1989 & 5 \\
\hline Sowers and Sandler ${ }^{67}$ & & $\mathrm{BH}$ perturbation theory; pressure explicit & 1991 & 6 \\
\hline Koutras et al. ${ }^{68}$ & Kou & modified HS equation; pressure explicit & 1992 & 15 \\
\hline Miyano $^{69}$ & Mi & empirical (MBWR); pressure explicit & 1993 & 33 \\
\hline Johnson et al. ${ }^{56}$ & Jo & empirical (MBWR); pressure explicit & 1993 & 33 \\
\hline Kolafa and Nezbeda ${ }^{57}$ & Ko & HS + virial + empirical; Helmholtz energy explicit & 1994 & 32 \\
\hline Mecke et al. ${ }^{70,71}$ & $\mathrm{Me}$ & HS + empirical; Helmholtz energy explicit & 1996 & 38 \\
\hline Sun and Teja ${ }^{72}$ & $\mathrm{Su}$ & empirical (MBWR); pressure explicit & 1996 & 33 \\
\hline Cuadros et al. ${ }^{73}$ & & WCA perturbation theory; Helmholtz energy explicit & 1996 & 5 \\
\hline Amadei et al. ${ }^{74}$ & & quasi Gaussian entropy theory; Helmholtz energy explicit & 1999 & 43 \\
\hline $\mathrm{Hess}^{75}$ & $\mathrm{He}$ & WCA reference + virial; pressure explicit & 1999 & 0 \\
\hline Boltachev and Baidakov ${ }^{76}$ & Bo & empirical + virial; pressure explicit & 2003 & 39 \\
\hline Paricaud $^{77}$ & $\mathrm{~Pa}$ & BH perturbation theory; Helmholtz energy explicit & 2006 & 34 \\
\hline Betancourt-Cardenas et al. ${ }^{78}$ & & BH perturbation theory; Helmholtz energy explicit & 2008 & 10 \\
\hline Quiñones-Cisneros et al. ${ }^{79}$ & Qui & empirical; pressure explicit & 2009 & 16 \\
\hline May and Mausbach 80,81 & Ma & empirical (MBWR); pressure explicit & 2012 & 33 \\
\hline Lafitte et al. ${ }^{42}$ & $\mathrm{La}$ & BH perturbation theory; Helmholtz energy explicit & 2013 & 62 \\
\hline Thol et al. ${ }^{37}$ & Th & empirical; Helmholtz energy explicit & 2016 & 67 \\
\hline van Westen and Gross $^{82}$ & vWe & BH perturbation theory; Helmholtz energy explicit & 2017 & 43 \\
\hline Gottschalk $^{83}$ & Go & empirical + virial & 20191 & 167 \\
\hline this work & th & BH perturbation theory; Helmholtz energy explicit & 2020 & 18 \\
\hline
\end{tabular}


The 20 LJ EOS for which an abbreviation is given in Table I were considered for the comparison in the present work. As only some of the LJ EOS are available in executable form or as source code, such a comparison requires programming and validation of the considered EOS. Besides the 20 LJ EOS studied here, two more LJ EOS ${ }^{73,78}$ were implemented in the course of this work, but the results reported in the respective publications could not be reproduced using the given equations and numerical values of the parameters. Hence, they were not considered for the comparison in this work.

Furthermore, in the course of the present work, misprints were identified in Refs. ${ }^{82}$ and $^{79}$. The corrections are reported in the Supplementary Material. Using these corrections, our implementations of the LJ EOS from Refs. ${ }^{79,82}$ yield results that are consistent with data reported in the respective publications.

All LJ EOS listed in Table I are either formulated explicitly in the pressure or the Helmholtz energy. The number of parameters that were adjusted to obtain a good fit of data from computer experiments varies between 0 and 177. The LJ EOS that were considered in the present work are classified here as follows: theoretically-based: Refs. ${ }^{29,42,77,82}$ and the LJ EOS from the present work; semi-theoretical: Refs. ${ }^{57,68,70,71,75,76}$; and empirical: Refs. ${ }^{12,28,30,37,56,69,72,79-81,83}$.

The most popular LJ EOS are those of Johnson et al. ${ }^{56}$, Nicolas et al. ${ }^{12}$, Kolafa and Nezbeda ${ }^{57}$, Cotterman et al. ${ }^{29}$, Lafitte et al. ${ }^{42}$, Ree ${ }^{28}$, and Mecke et al. ${ }^{70,71}$ (sorted by their number of citations; for more information, see the Supplementary Material).

The first LJ EOS were published soon after molecular simulations became feasible by 'computing machines, ${ }^{3-6}$. The oldest LJ EOS considered in the present work is that of Nicolas et al. ${ }^{12}$ from 1979, which is a modified Benedict-Webb-Rubin (MBWR) type EOS ${ }^{84,85}$. That LJ EOS has been re-parametrized several times, e.g. from Adachi et al. ${ }^{30}$ Miyano $^{69}$, Johnson et al. ${ }^{56}$, Sun and Teja ${ }^{72}$, and May and Mausbach ${ }^{80,81}$, of which the parametrization of Johnson et al. ${ }^{56}$ is by far the most frequently used. The oldest LJ EOS of the 'theoretically-based' type considered here is that of Cotterman et al. ${ }^{29}$ from 1986 . Both Kolafa and Nezbeda $a^{57}$ and Mecke et al. ${ }^{70,71}$ proposed semi-theoretical LJ EOS in the 90s that became popular. Several theoretically-based LJ EOS have been developed in the recent years, e.g. those of Paricaud ${ }^{77}$, Lafitte et al. $^{42}$, and van Westen and Gross ${ }^{82}$. But also new empirical LJ EOS have become available recently, e.g. those of Thol et al. ${ }^{37}$ and Gottschalk $^{83}$. Further semi-theoretical LJ EOS that are considered in the present work are 
those of Boltachev and Baidakov ${ }^{76}$, Quiñones-Cisneros et al. ${ }^{79}$, Koutras et al. ${ }^{68}$, and Hess ${ }^{75}$; and the empirical LJ EOS of $R e e^{28}$.

Theoretically-based LJ EOS are usually either built on the perturbation theory of Barker and Henderson ${ }^{86,87}(\mathrm{BH})$ or that of Weeks-Chandler-Andersen ${ }^{88}$ (WCA). The LJ EOS of Song and Mason ${ }^{65}$ is of the WCA type, those of Cotterman et al. ${ }^{29}$, Paricaud ${ }^{77}$, Lafitte et $a .^{42}$, van Westen and Gross $^{82}$, and the PC-SAFT monomer are of the BH type. Van Westen and Gross ${ }^{82}$ also proposed a WCA type LJ EOS and found it equally precise as their $\mathrm{BH}$ type LJ EOS. Only the latter is employed here for comparison. Perturbation theory is expected to be a good approximation for state points for which the influence of the attractive forces is not particularly strong, i.e. high temperatures ${ }^{86,89}$.

The theoretically-based LJ EOS apply different formulations for the hard sphere reference term, e.g. the LJ EOS of Cotterman et al. ${ }^{29}$, Paricaud ${ }^{77}$, van Westen and Gross $^{82}$, and Lafitte et al. ${ }^{42}$ use the hard sphere equation of Carnahan and Starling ${ }^{90}$, whereas the PCSAFT monomer uses that of Boublik ${ }^{91}$ and Mansoori et al. ${ }^{92}$. Also the semi-theoretical LJ EOS of Mecke et al. ${ }^{70,71}$ employs the Carnahan and Starling ${ }^{90}$ equation for the description of the hard sphere Helmholtz energy. The semi-theoretical LJ EOS of Kolafa and Nezbeda ${ }^{57}$ employs a slightly modified version of the Carnahan and Starling ${ }^{90}$ equation. The LJ EOS of Koutras et al. ${ }^{68}$ is based on an empirically modified version of the Carnahan and Starling ${ }^{90}$ equation.

The BH type LJ EOS of Cotterman et al. ${ }^{29}$, Paricaud $^{77}$, Lafitte et al. ${ }^{42}$, and van Westen and Gross $^{82}$ as well as the PC-SAFT monomer use slightly differing formulations for the temperature-dependent diameter for the modeling of the softness of the repulsive interactions $^{86,89}$. The PC-SAFT monomer model, which was re-parametrized in the present work, uses a simplified version of the temperature-dependent diameter, cf. Eq. (24) in the Appendix.

The theoretically-based LJ EOS of Cotterman et al. ${ }^{29}$, Paricaud ${ }^{77}$, and the PC-SAFT monomer model are developed up to the second order perturbation term. The LJ EOS of Lafitte et al. ${ }^{42}$ and van Westen and Gross $^{82}$ are formulated up to third and fourth order perturbation terms, respectively.

Also the perturbation terms are designed differently in the considered theoretically-based LJ EOS: the LJ EOS of Cotterman et al. ${ }^{29}$ and the PC-SAFT monomer model are simplified and developed in a series expansion in the packing fraction, whereas the LJ EOS 
Paricaud ${ }^{77}$, Lafitte et al. ${ }^{42}$, and van Westen and Gross $^{82}$ use an analytic function for the radial distribution function of the hard sphere system.

All LJ EOS considered here were fitted to computer experiment data (except that of Hess $^{75}$, which has no adjustable parameter). However, the data that was used generally differs. Furthermore, in many cases the training data set, the objective functions, and weights were not disclosed in the publications. Most LJ EOS were parametrized using $p v T$ data - often in combination with internal energy data. In some cases also data on the thermal virial coefficients were used for the parametrization - mainly for empirical LJ EOS. The LJ EOS of Thol et al..$^{37}$, which is explicit in the Helmholtz energy $a$, was parametrized directly using computer experiment data of $a$ and its density and inverse temperature derivatives. Most theoretically-based LJ EOS ${ }^{29,42,82}$ are directly parametrized using computer experiment data of the perturbation term. The data type used for the parametrization of each LJ EOS is summarized in the Supplementary Material.

In some publications in which new LJ EOS are proposed (cf. Table I), an estimated range of validity of the LJ EOS is reported (which is usually given for the density and temperature). Since this range of validity is mostly based only on estimates and in some cases only described ambiguously, we compare all LJ EOS to the same computer experiment database $^{11}$, disregarding the eventual statements on the range of validity to ensure a fair comparison. We find that the reported range of validity of many LJ EOS is either far too optimistic or pessimistic. Information on the reported range of validity of each LJ EOS is given in the Supplementary Material.

\section{EVALUATION OF LJ EOS}

Table VII in the Appendix summarizes the reference database adopted from Ref. ${ }^{11}$, which contains approximately 35,000 data points. These data are also available electronically in the Supplementary Material, such that the tests of the different LJ EOS that were carried

out in the present work can easily be reproduced and the approach can be extended to the evaluation of new LJ EOS. Data points that were identified as outliers in Ref. ${ }^{11}$ were discarded for the comparison in the present work. The reference data was not differentiated here regarding the source of the data points. However, also that information can be retrieved from the electronic Supplementary Material. Moreover, the reference data was produced 
over the last decades by a large number of different source codes and will in general differ in system size, simulation time, cut-off radius, time step, the employed long-rang correction scheme etc. Hence, finite-size effects may have been treated differently for the production of that large entity of reference data. See Ref. ${ }^{11}$ for a detailed discussion of the reference data and the algorithm used for the identification of gross outliers.

As the results from computer simulations are subject to both systematic and statistical errors $^{11,93,94}$, the true value of a given property of the LJ fluid at a given state point is only known with some uncertainty. That uncertainty depends on the property that is investigated as well as on the state point. Furthermore, the uncertainty can only be estimated and, depending on the way this estimate is carried out, different numbers for the uncertainty are obtained. We do not re-enter into the discussion of the quality of the reference data and simply refer to Ref. ${ }^{11}$. The goal of any description of properties of the LJ fluid by an EOS must be to describe the primary data within their uncertainty. Ideally, this should be fulfilled for all properties of interest in the entire fluid region.

The systematic approach for the comparison of the LJ EOS that was applied in the present work is briefly described in the following. The performance of each LJ EOS $i$ was examined for different thermophysical properties $j$ and fluid regions $k$ regarding data points $l$ from computer experiments.

The relative deviation $\delta Y_{i j k l}$ of a result $Y_{i j k l}$ from an LJ EOS $i$ from the corresponding computer experiment $Y_{\text {ref }, j k l}$ for a given property $j$ in a given fluid region $k$ is defined as

$$
\delta Y_{i j k l}=\frac{Y_{i j k l}-Y_{\mathrm{ref}, j k l}}{Y_{\mathrm{ref}, j k l}} .
$$

The complete set of numeric values $Y_{i j k l}$ computed for each data point $l$ from the database ${ }^{11}$ for each LJ EOS $i$ is provided in the electronic Supplementary Material together with the corresponding reference value $Y_{\text {ref }, j k l}$. Where available, also the statistical uncertainties from the computer experiment data are given.

The absolute average deviation $\mathrm{AAD}_{i j k}$ of a given LJ EOS $i$ for a thermophysical property $j$ in a fluid region $k$ is defined as

$$
\mathrm{AAD}_{i j k}=1 / N_{j k} \sum_{l=1}^{N_{j k}}\left|\delta Y_{i j k l}\right|,
$$

where $N_{j k}$ is the number of reference data points for the respective property $j$ in the fluid 
region $k$. Hence, the $\mathrm{AAD}_{i j k}$ quantifies the performance of an LJ EOS for a certain property $j$ in a certain fluid region $k$. The overall performance of an LJ EOS $i$ for a given property $j$ was evaluated using

$$
\mathrm{AAD}_{i j}=1 / N_{\text {tot }} \sum_{l=1}^{N_{\text {tot }}}\left|\delta Y_{i j l}\right|,
$$

where $N_{\text {tot }}$ is the number of data points available for a given property $j$. For clarity and simplicity we sometimes refer to $\mathrm{AAD}_{i j}$ for a given property $j$ simply as $\mathrm{AAD}_{j, \text { total }}$ in the following text, when it is clear which LJ EOS $i$ is meant.

The classification into different fluid regions $k$ is only used for homogeneous state data points specified as $Y=Y(T, \rho)$. Fig. 1 shows the eight fluid regions that were used in the present work for the classification of $Y=Y(T, \rho)$ data. Table II summarizes the conditions for each region. The binodal was adopted from an analytic correlation of Stephan et $a .^{11}$, and the freezing line from Köster et al. ${ }^{21}$. The line separating data in the high density liquid and high density supercritical region was chosen as $95 \%$ of the density of the freezing line reported by Köster et al. ${ }^{21}$. Since only very few computer experiment data has been reported for the unstable region ${ }^{11,69,95}$ obtained from specialized techniques and the location of the spinodal is only known with large uncertainty (see below), the metastable and unstable region were combined for the comparison to a single region enclosed by the binodal. An eighth characteristic region was defined at extreme temperatures $T>6$, i.e. the supercritical and high density supercritical region end at $T=6$. All data points from the database ${ }^{11}$ of the type $Y=Y(T, \rho)$ were assigned to one of these eight regions. The allocation of each data point is reported in the electronic Supplementary Material. Data points that are specified as $Y=Y(T)$, namely VLE properties, virial coefficients, and spinodal data, belong to individual fluid regions.

For each LJ EOS $i$, the obtained $\mathrm{AAD}_{i j k}$ for each property $j$ and each region $k$ and $\mathrm{AAD}_{i j}$ are reported in Tables III - V and are discussed in the following sections. 


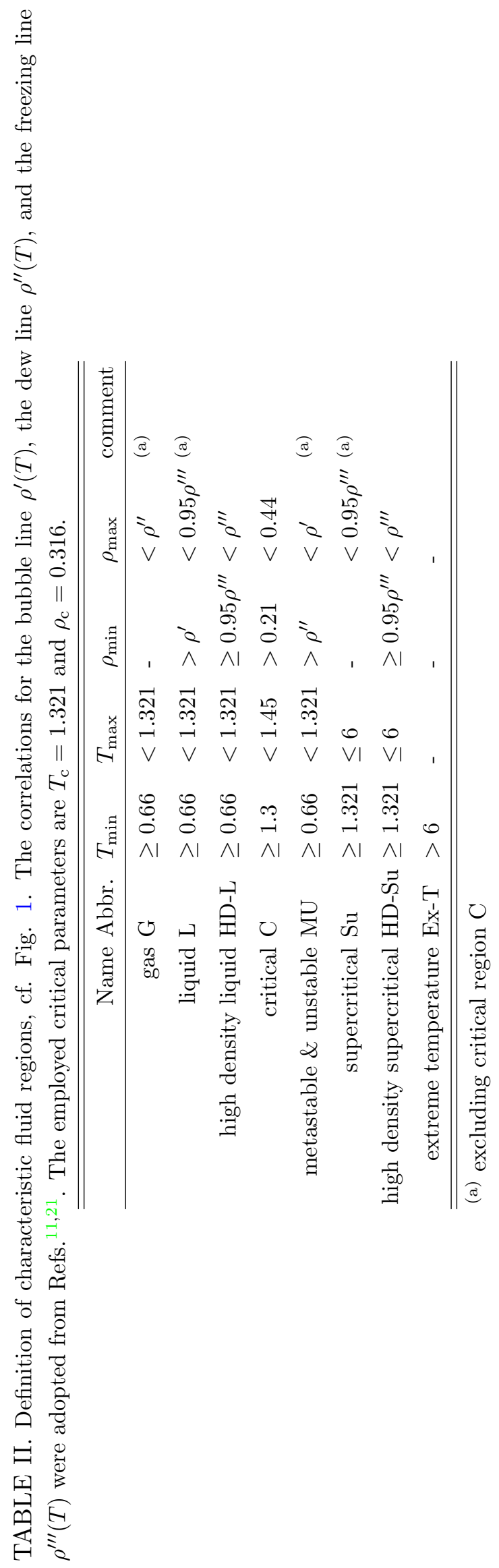




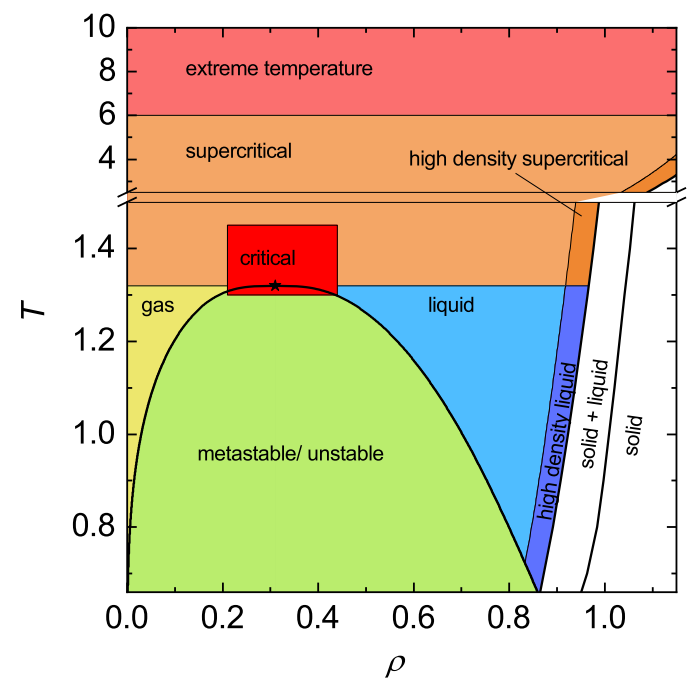

FIG. 1. Definition of characteristic fluid regions (colored) of the LJ fluid used for the evaluation and comparison of LJ EOS in the present work: metastable/ unstable (MU), gas (G), liquid (L), critical (C), supercritical (Su), high density liquid (HD-L), high density supercritical (HD-Su), and extreme temperature (Ex-T). Details on the boundaries of the regions are given in Table II. Solid states and the melting region were not considered in the present study.

\section{Vapor-Liquid Equilibrium}

The vapor-liquid equilibrium of the LJ fluid has been investigated numerous times ${ }^{10,11,18,78,96-135}$. Seven of these 45 data sets were found to be significantly more precise and accurate than the remaining ${ }^{11}$. Only these seven best data sets were used here for the comparison, cf. Table VII in the Appendix. However, also these seven data sets contain eight clear outliers ${ }^{11}$ that were discarded for the present investigation. The mutual agreement of these seven data sets was estimated to be $\pm 1 \%$ for the vapor pressure, $\pm 0.2 \%$ for the saturated liquid density, $\pm 1 \%$ for the saturated vapor density, and $\pm 0.75 \%$ for the enthalpy of vaporization - excluding the region close to the critical point ${ }^{11}$. Furthermore, empirical correlations of these seven best datasets for $p^{s}, \Delta h_{\mathrm{v}}, \rho^{\prime}$, and $\rho^{\prime \prime}$ have been proposed in Ref. ${ }^{11}$. The relative deviations of the VLE properties are shown in Fig. 2 (vapor pressure $p^{\mathrm{s}}$ and the enthalpy of vaporization $\Delta h_{\mathrm{v}}$ ) and in Fig. 3 (saturated liquid and vapor density $\rho^{\prime}$ and $\rho^{\prime \prime}$, respectively). The baselines are the empirical correlations from Ref. ${ }^{11}$, other lines are the LJ EOS, and symbols indicate reference data from the computer experiments. Table III lists the $\mathrm{AAD}_{j, \text { total }}$ as defined by Eq. (4) obtained from the reference VLE data up to $T=$ 1.29 and each of the 20 considered LJ EOS for $j=\rho^{\prime}, \rho^{\prime \prime}, p^{\mathrm{s}}, \Delta h_{\mathrm{v}}$. The VLE computed from each of the considered LJ EOS are reported in the electronic Supplementary Material. 

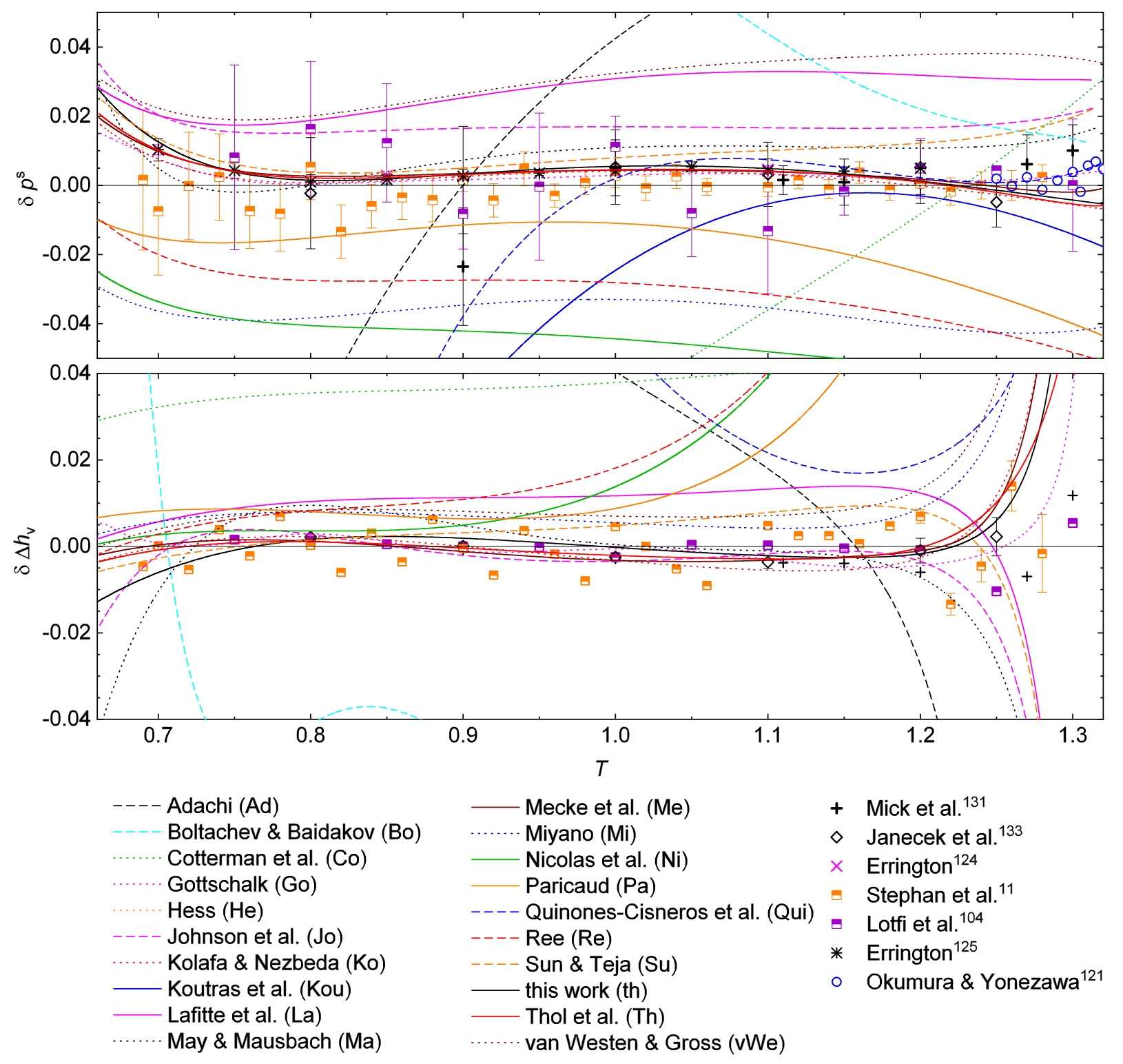

FIG. 2. Comparison of results from 20 LJ EOS with reference data: relative deviation of the vapor pressure (top) and enthalpy of vaporization (bottom). The base line indicates the respective correlation of computer experiment data of Stephan et al. ${ }^{11}$. The other lines are results from the LJ EOS; symbols are data from computer experiment.

The LJ EOS of Thol et al. ${ }^{37}$, Mecke et al. ${ }^{70,71}$, Kolafa and Nezbeda ${ }^{57}$, Gottschalk ${ }^{83}$, and from the present work show the best agreement with the VLE simulation results. Nevertheless, the LJ EOS of Thol et al. ${ }^{37}$, Mecke et al. ${ }^{70,71}$, Gottschalk ${ }^{83}$, and the LJ EOS from the present work exhibit minor systematic deviations from the simulation results in the saturated liquid density at $T>1$. Only the LJ EOS of Kolafa and Nezbeda ${ }^{57}$ captures this trend correctly. 


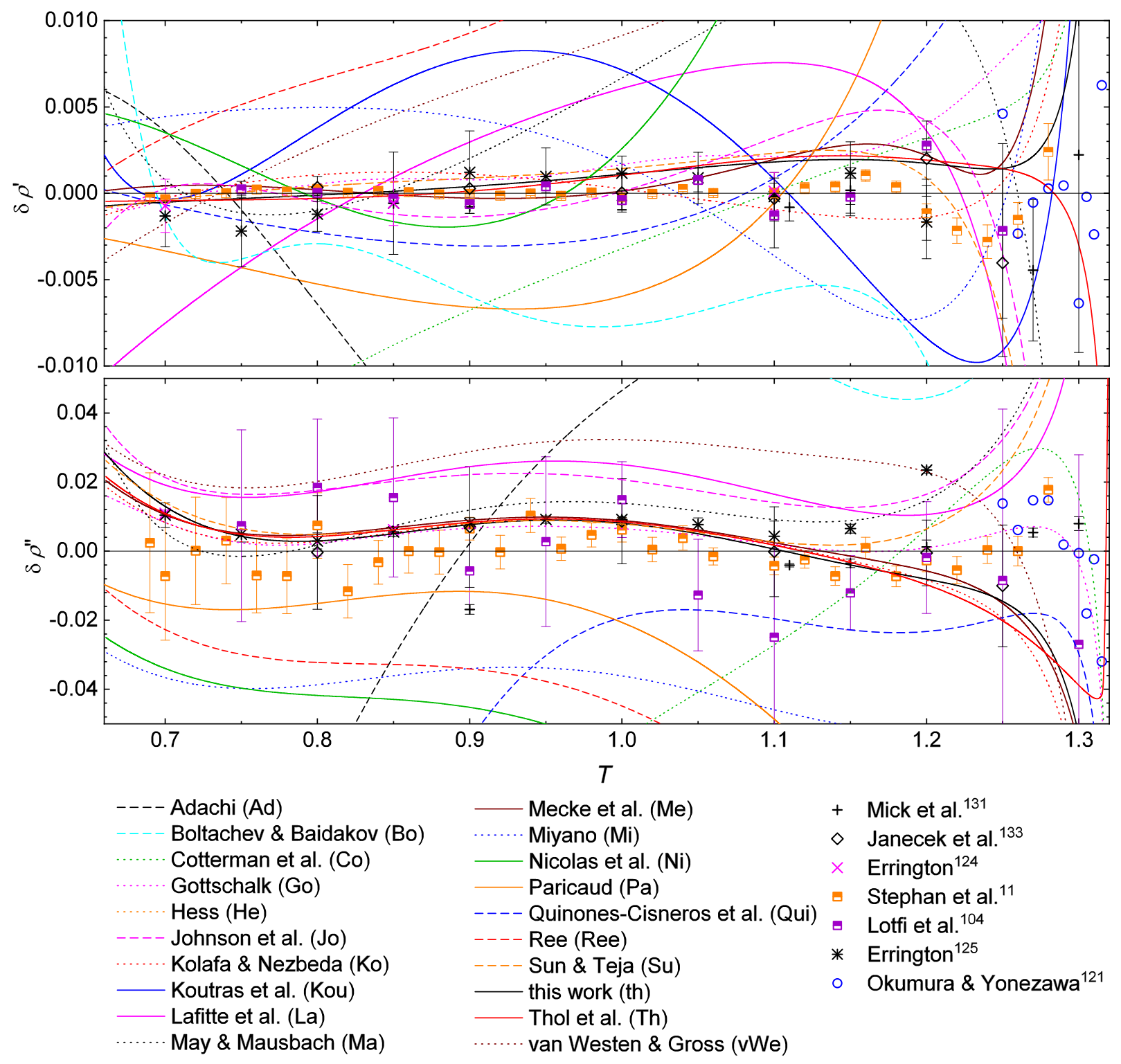

FIG. 3. Comparison of results from 20 LJ EOS with reference data: relative deviation of the saturated densities: liquid density (top) and vapor density (bottom). The base line indicates the correlation of computer experiment data of Stephan et al. ${ }^{11}$. The other lines are results from the LJ EOS; symbols are data from computer experiment.

The LJ EOS of Johnson et al. ${ }^{56}$, May and Mausbach ${ }^{80,81}$, Sun $\&$ Teja ${ }^{72}$, Lafitte et al. ${ }^{42}$, and van Westen and Gross $^{82}$ also give a good description of the VLE properties of the LJ fluid. However, the LJ EOS of Lafitte et al. ${ }^{42}$, van Westen and Gross ${ }^{82}$, and Johnson et al. ${ }^{56}$ exhibit distinct systematic deviations from the reference data of the vapor pressure and the saturated densities. The LJ EOS of May and Mausbach ${ }^{80,81}$ exhibits noticeable deviations regarding the saturated liquid density at $T>1$. 
Particularly large deviations from the computer experiment VLE data are found for the LJ EOS of Nicolas et al. ${ }^{12}$, Miyano ${ }^{69}$, Boltachev and Baidakov ${ }^{76}$, Adachi et al. ${ }^{30}$, Cotterman et al. ${ }^{29}$, Paricaud ${ }^{77}$, Hess ${ }^{75}$, Koutras et al. ${ }^{68}$, Ree ${ }^{28}$, and Quiñones-Cisneros et al. ${ }^{79}$.

All considered LJ EOS are fully analytic and thereby exhibit classical scaling behavior, e.g. quadratic convergence of the saturated densities near the critical point ${ }^{136-138}$. This, and the fact that the investigated LJ EOS have significantly differing critical temperatures (see following section), leads to the diverging deviations of the VLE properties in Figs. 2 and 3 in the vicinity of the critical point. Also the scattering of the computer experiment VLE data significantly increases in the vicinity of the critical point and, accordingly, only very few consistent data points are available in that region ${ }^{11}$.

For each VLE property $j$, the lowest absolute average deviation $\mathrm{AAD}_{i j}$ among the considered LJ EOS $i$ in Table III is in good agreement with the stated mutual agreement of the best computer experiment data, which indicates that the lowest absolute average deviation values are in fact good guesses for the accuracy of the reference data. 


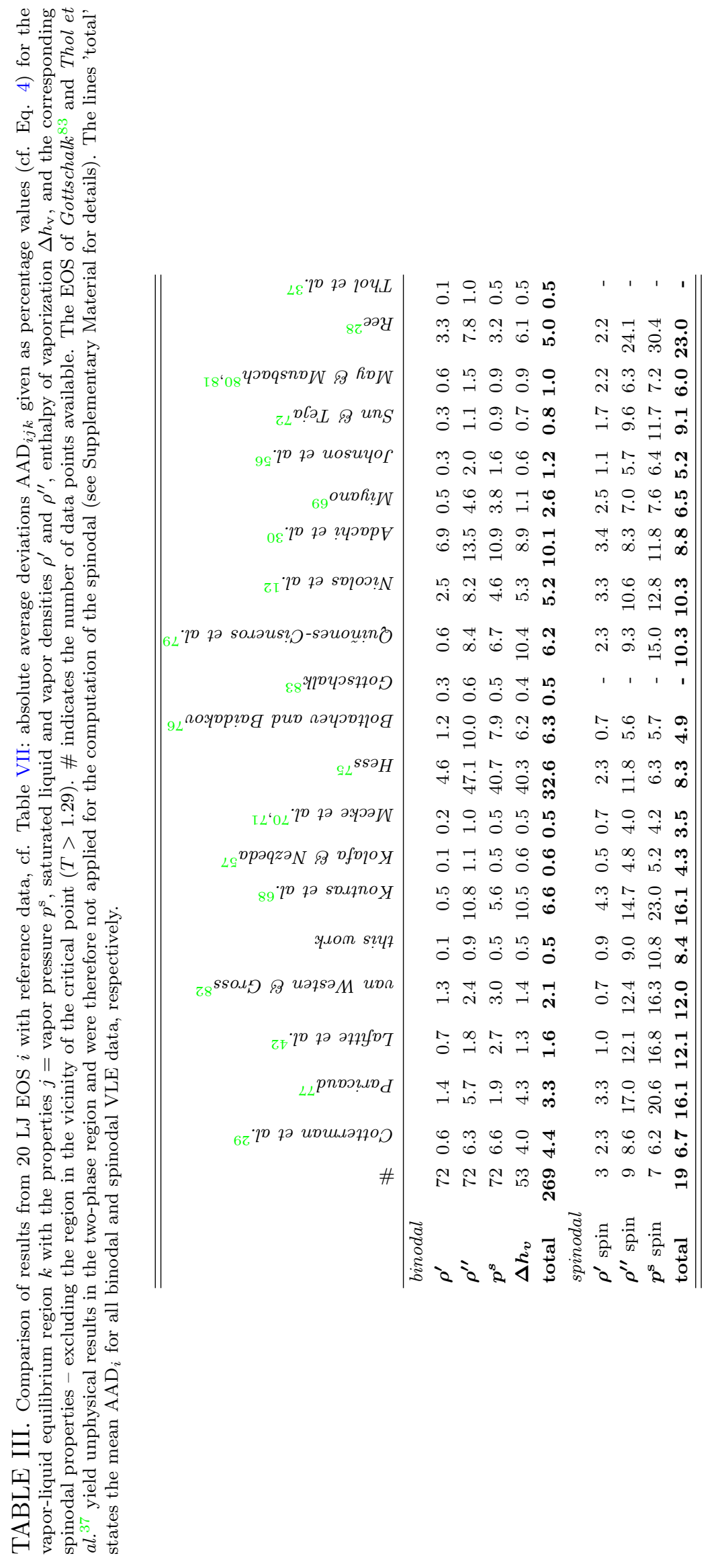




\section{Critical Point}

Critical data derived from computer experiment data of the LJ fluid have been reported several times in the literature $8,28,61,67,78,99,100,104,108,113,116,118,119,121,139-152$. They were summarized and assessed in Ref. ${ }^{11}$. The critical data of the LJ fluid were thereby estimated to be $T_{\mathrm{c}}=1.321 \pm 0.007$ and $\rho_{\mathrm{c}}=0.316 \pm 0.005$ and $p_{\mathrm{c}}=0.129 \pm 0.005^{11}$. These values are referred to as 'computer experiment' critical data in the following. They were obtained from a large number of critical data for the LJ fluid reported in the literature and cross-evaluation with the most reliable VLE data available in the vicinity of the critical point ${ }^{11}$. The given uncertainties were estimated from the mutual agreement of the reported data. The uncertainties for example account for differences in the extrapolation to infinite systems, different cut-off radius, etc.

The numeric values of the critical data computed from the LJ EOS are reported in the Supplementary Material. Fig. 4 shows the comparison of the critical data obtained from the LJ EOS and the computer experiment values with their uncertainties. The critical data from the LJ EOS scatter in a large range compared to the uncertainty of the critical data from the computer experiments. Six LJ EOS (Refs. ${ }^{12,68,69,75,77,82}$ ) have particularly large deviations from the computer experiment critical point. Many LJ EOS show fair agreement with the critical density from the computer experiments, but a significant overestimation of the critical temperature and pressure is found in most cases, which is due to the scaling behavior of the $\operatorname{EOS}^{136-138}$. The critical pressure obtained from the vast majority of LJ EOS lies within a narrow band in the $p-T$ diagram. The width of that band is similar to the estimated uncertainty of the critical pressure from the computer experiments $\delta p_{\mathrm{c}}= \pm 0.005$. Hence, the deviation of the critical point computed from the LJ EOS is mainly a result of the mismatching critical temperature - as expected from the scaling behavior.

The critical parameters obtained from the LJ EOS of Johnson et al. ${ }^{56}$, Lafitte et al. ${ }^{42}$, May and Mausbach ${ }^{80,81}$, and Thol et al. ${ }^{37}$ are in excellent agreement with the computer experiment, which is surprising considering the fact that they are analytic EOS and therefore should not be accurate in the vicinity of the critical point. Lafitte et al. ${ }^{42}$ assume that the applied third order perturbation term is responsible for the relatively low critical tem-

perature of their LJ EOS. However, the LJ EOS of van Westen and Gross ${ }^{82}$ significantly overestimates the critical temperature of the computer experiment, but also includes higher 

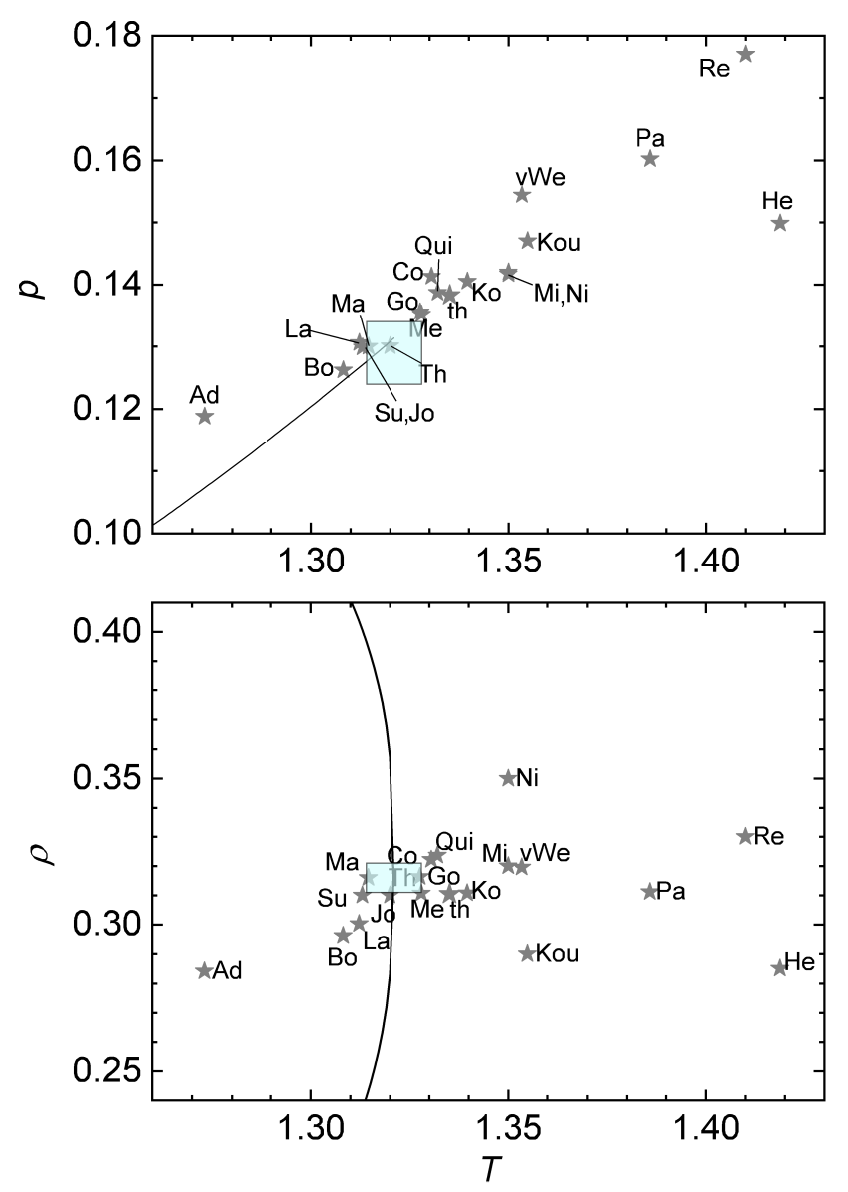

FIG. 4. Critical point of the LJ fluid. Top: critical pressure over temperature. Bottom: critical density over temperature. Each combination of a star \& abbreviation indicates the critical parameters from an LJ EOS. The abbreviations are the same as those in Table I. The blue shaded area indicates the critical parameters from computer experiments and their uncertainties ${ }^{11}$. The solid lines indicate the correlations of the respective VLE properties from Stephan et al. ${ }^{11}$. The numeric values of the critical parameters obtained from the LJ EOS are reported in the Supplementary Material.

order perturbation terms. Despite its good results for the critical data, the LJ EOS of Thol et $a l .{ }^{37}$ has a relatively poor performance for state points in the vicinity of the critical point, even crossing isotherms are observed (see next section). This poor performance is probably also related to the presence of a second spurious critical point at $T_{\mathrm{c}}=1.30351, \rho_{\mathrm{c}}=0.31027$, $p_{\mathrm{c}}=0.12124$ obtained from the LJ EOS of Thol et al. ${ }^{37}$, see also Refs. ${ }^{83,153}$.

The critical parameters obtained from the LJ EOS of Cotterman et al. ${ }^{29}$, Kolafa and Nezbeda ${ }^{57}$, Mecke et al. ${ }^{70,71}$, Quiñones-Cisneros et al. ${ }^{79}$, Gottschalk ${ }^{83}$, and the LJ EOS from the present work are generally in reasonable agreement with critical data from the computer simulations, but they overestimate the critical temperature $T_{\mathrm{c}}$ by about $0.01 \ldots 0.02$. The 
LJ EOS of Paricaud ${ }^{77}, \mathrm{Hess}^{75}$, and $\mathrm{Ree}^{28}$ overestimate the critical temperature by about $0.06 . .0 .09$.

\section{Isotherms in the Two-Phase Region and Spinodal}

Equations of state should exhibit only a single van der Waals loop in the two-phase region. Multiple oscillations of isotherms are prohibitive for calculating interfacial properties, e.g. with density gradient theory ${ }^{154-158}$. Furthermore, isotherms in the two-phase region can show artificial undulations around the general course of the van der Waals loop, which have a smaller amplitude than that of the van der Waals loop. Another problem that can occur are crossing isotherms (multiplicity).

In this section, the behavior of the LJ EOS is characterized regarding the behavior of isotherms in the two-phase region in the $p-\rho$ diagram and the spinodal. The results from the quantitative comparison of the LJ EOS with computer experiment data of the type $Y=Y(T, \rho)$ for state points between the binodals with LJ EOS are discussed in the subsequent section.

The isotherms in the $p-\rho$ diagram of all 20 considered LJ EOS were examined in the temperature range $T=0.5 \ldots 1.32$ regarding the following features in the two-phase region: the number of zero crossings of $\mathrm{d} p / \mathrm{d} \rho=0$ and $\mathrm{d}^{2} p / \mathrm{d} \rho^{2}=0$; the results are summarized in Fig. 5. The numeric values of four isotherms in the two-phase region are reported in the electronic Supplementary Material.

Most theoretically-based LJ EOS show none of the problems mentioned above in the two-phase region, whereas most empirical and semi-theoretical LJ EOS show at least some artifacts in the two-phase region. The LJ EOS of Gottschalk ${ }^{83}$ and Thol et al. ${ }^{37}$ exhibit multiple minima and maxima in a wide temperature range, cf. Fig. 5. The LJ EOS of Thol et al. ${ }^{37}$ exhibits artificial oscillations up to extreme negative and positive pressures (details are given in the Supplementary Material), which results in a crossing of isotherms ${ }^{34-36}$ not only in the two-phase region but also the supercritical region. For both the LJ EOS of Gottschalk $^{83}$ and Thol et al. ${ }^{37}$ for some temperatures above the triple point temperature, two subcritical isotherms may cross each other (in the two-phase region) as well as a subcritical and a supercritical isotherm may cross (above the critical temperature). For the LJ EOS

of Boltachev and Baidakov ${ }^{76}$, supercritical isotherms above $T \gtrsim 2$ exhibit a maximum and 

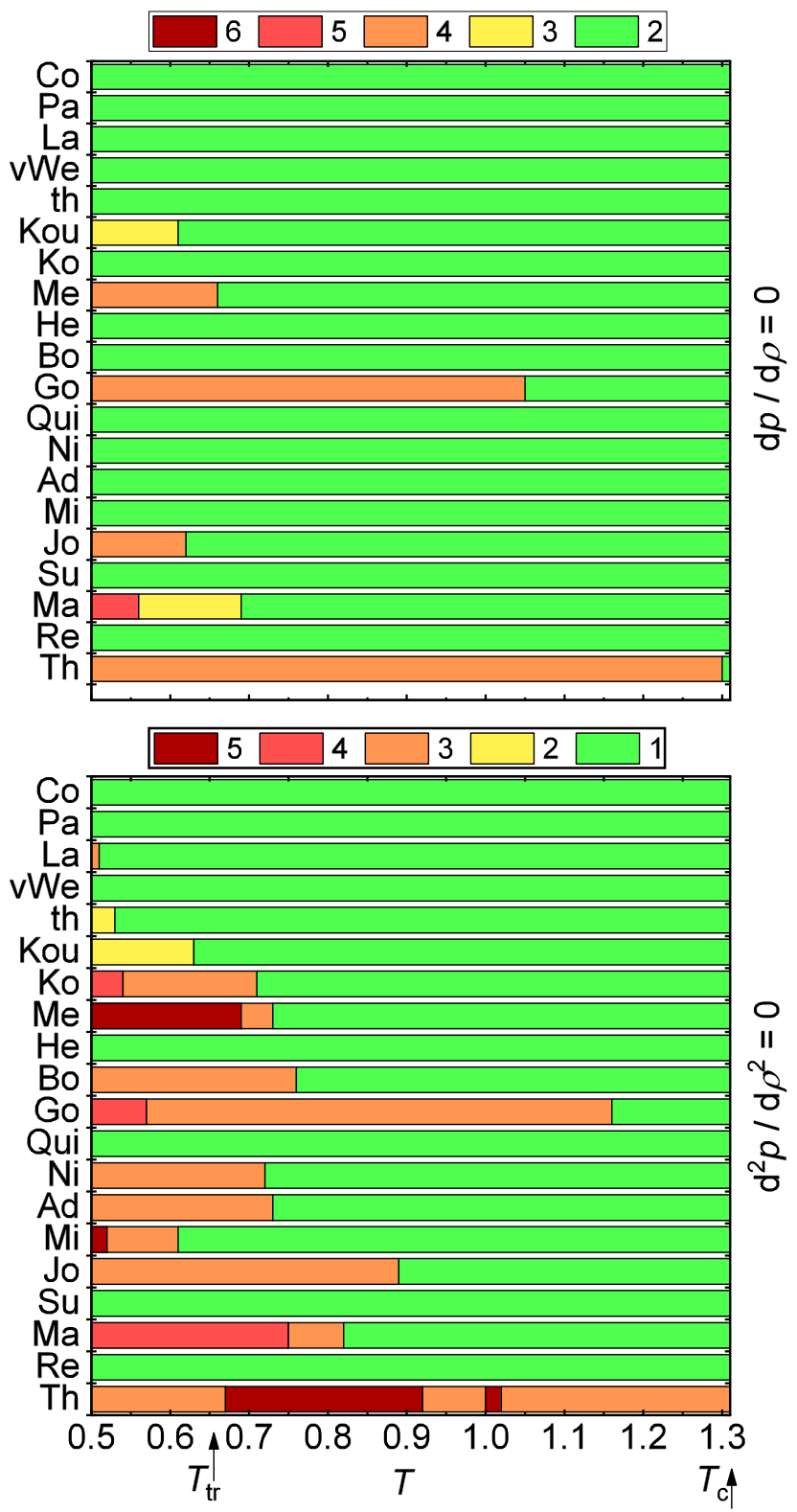

FIG. 5. Number of zero crossings of the first and second derivative of the $p-\rho$ isotherms $(\mathrm{d} p / \mathrm{d} \rho=0$ and $\left.\mathrm{d}^{2} p / \mathrm{d} \rho^{2}=0\right)$ in the two-phase region as a function of the temperature for all 20 considered LJ EOS. The top panel indicates the number of $\mathrm{d} p / \mathrm{d} \rho=0$ at a given temperature; the bottom panel indicates the number of $\mathrm{d}^{2} p / \mathrm{d} \rho^{2}=0$. The desired values are: two for the upper panel and one for the lower panel and are indicated in green. The abbreviations for the LJ EOS are the same as in Table I.

minimum that also yields a crossing of isotherms. For $T \gtrsim 2.6$, supercritical isotherms of the LJ EOS of Boltachev and Baidakov ${ }^{76}$ may even cross the vapor-liquid phase boundary.

The LJ EOS of Refs. ${ }^{56,70,71,80,81}$ exhibit multiple extrema only in the vicinity or below the triple point temperature. For the LJ EOS of Boltachev and Baidakov ${ }^{76}$, isotherms exhibit 
a second (unphysical) maximum in the homogeneous high density region, which yields large deviations from reference data in that region (see following section).

Several LJ EOS also exhibit undulations of the isotherms between the binodals in the $p-\rho$ diagram. This leads to the fact that the isotherms in the $p-\rho$ diagram of the LJ EOS of Johnson et al. ${ }^{56}$, May and Mausbach ${ }^{80,81}$, Nicolas et al. ${ }^{12}$, Mecke et al. ${ }^{70,71}$, Boltachev and Baidakov ${ }^{76}$, Kolafa and Nezbeda ${ }^{57}$, and Adachi et al. ${ }^{30}$ exhibit a single van der Waals loop, but multiple turning points. The isotherms of the LJ EOS of Miyano ${ }^{69}$, Lafitte et al. ${ }^{42}$, Koutras et $a .^{68}$, and the LJ EOS from the present work exhibit such turning points only below the triple point temperature, cf. Fig. 5 - bottom.

For most of the investigated LJ EOS, the undulations of the isotherms in the two-phase region decrease with increasing temperature and vanish upon approaching the critical point (see the Supplementary Material). The only exception is the LJ EOS of Thol et al. ${ }^{37}$, which exhibits large oscillations up to the critical temperature.

LJ EOS with isotherms with a single and smooth van der Waals loop in the entire considered temperature range (cf. Fig. 5) are those of Cotterman et al. ${ }^{29}$, Paricaud ${ }^{77}$, Quiñones-Cisneros et al. ${ }^{79}$ Hess $^{75}$, Ree $^{28}$, and van Westen and Gross ${ }^{82}$. The isotherms of the LJ EOS of Sun and Teja ${ }^{72}$, Lafitte et $a .^{42}$, and the LJ EOS from the present work exhibit very small undulations between the binodals above the triple point - without producing a second turning point in the isotherms (see electronic Supplementary Material).

Recently, Alsaifi ${ }^{153}$ carried out a study on the behavior of six empirical and semi-

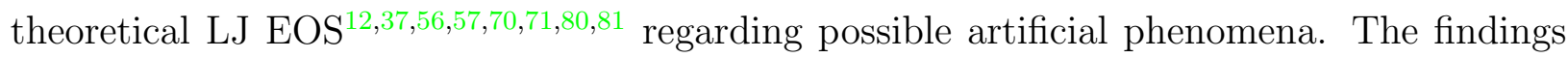
for these six LJ EOS are in line with the results from the present work.

Data derived from computer experiments for the spinodal of the LJ fluid have been reported by Baidakov et al. ${ }^{122}$ and Linhart et al. ${ }^{159}$. This data was compared with the spinodals computed from the 20 LJ EOS that were considered here. The bottom section of Table III reports the obtained absolute average deviations. In the assessment of the deviations, it must be considered that only few reference data points are available that are also subject to strong scattering (see Supplementary Material).

The LJ EOS of Mecke et al. ${ }^{70,71}$, Kolafa and Nezbeda ${ }^{57}$, Boltachev and Baidakov ${ }^{76}$, and Johnson et $a l .{ }^{56}$ yield the best agreement with the spinodal reference data. Due to the artificial oscillatory behavior of the isotherms from the LJ EOS of Gottschalk ${ }^{83}$ and Thol et $a l .{ }^{37}$, no unique spinodal can be obtained from those LJ EOS. We computed the spinodal 
for the first maximum and minimum and report the results in the electronic Supplementary Material for completeness.

\section{Homogeneous Fluid States}

In the present section, the performance of the LJ EOS for describing thermodynamic properties of homogeneous fluid state points is discussed, which are calculated as $Y=$ $Y(T, \rho)$. The considered states are grouped in the fluid regions $k$ shown in Fig. 1 . The reference data of each property $j$ of that type (cf. Table VII in the Appendix) was compared with the corresponding results of each of the LJ EOS $i$. The obtained numbers for $\mathrm{AAD}_{i j k}$ and $\mathrm{AAD}_{i j}$, cf. Eq. (3) and (4), are reported in Tables IV - V. Since several LJ EOS have extremely large deviations from the reference data (many orders of magnitude) in the extreme temperature region $k=$ Ex-T, which would lead to a meaningless total $\mathrm{AAD}_{i j}$, the Ex-T results were not comprised in the $\mathrm{AAD}_{i j}$ calculation but are reported separately in Table $\mathrm{V}$ instead. 


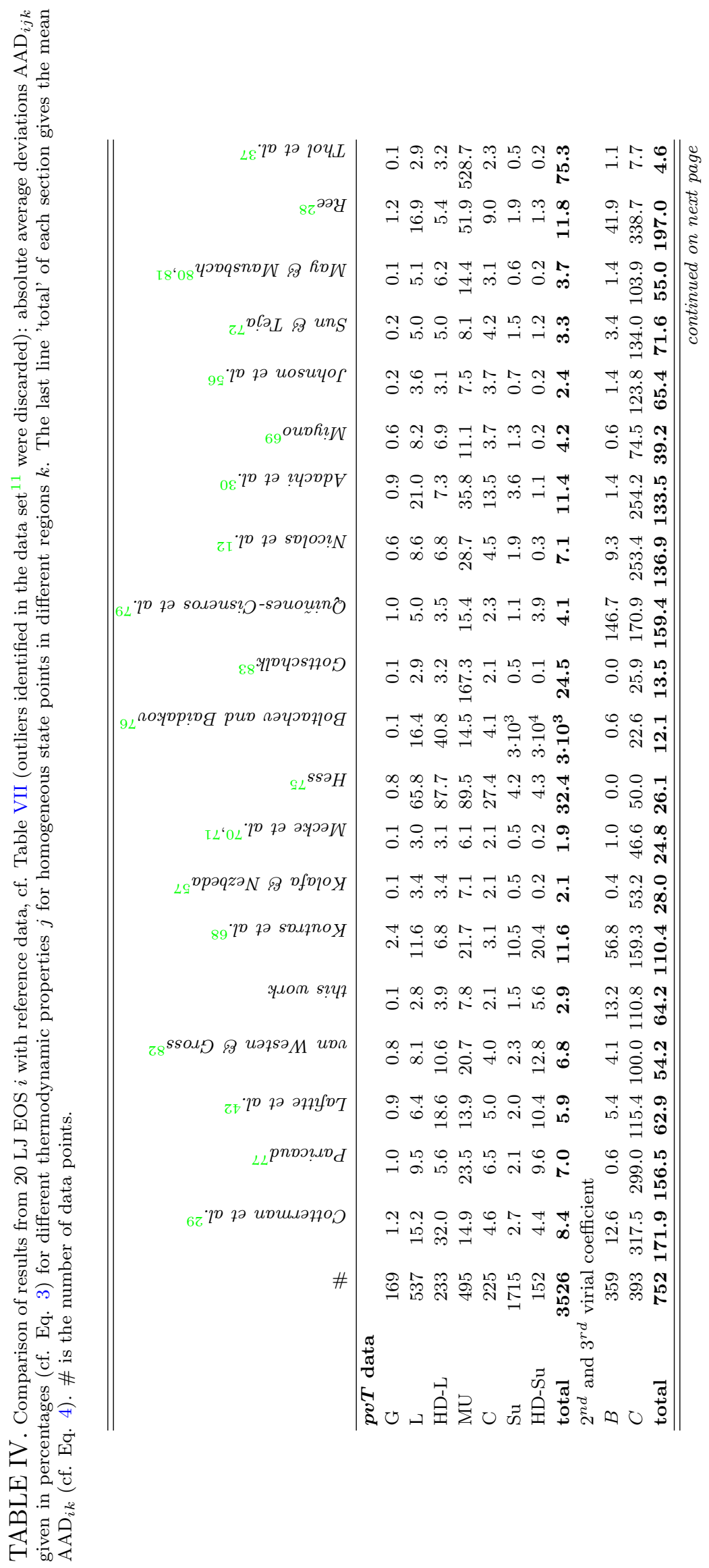




\begin{tabular}{|c|c|c|c|}
\hline$\angle \varepsilon^{\circ} l^{D}$ да $10 Y_{L}$ & 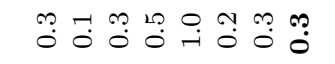 & 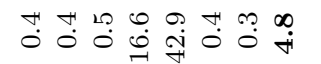 & 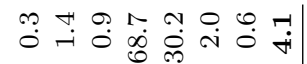 \\
\hline $8 \tau^{\partial ว y}$ & 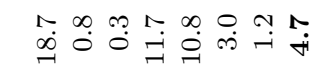 & 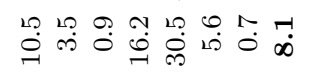 & 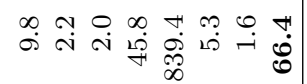 \\
\hline 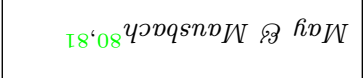 & 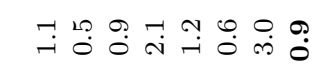 & 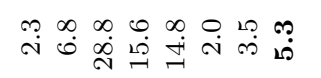 & 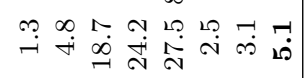 \\
\hline 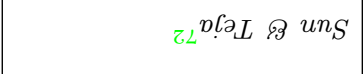 & 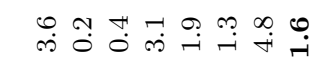 & 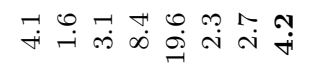 & 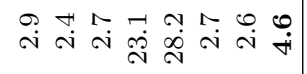 \\
\hline $99^{\prime} l^{D}$ ға иовичо & 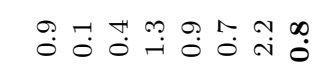 & 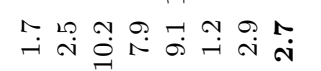 & 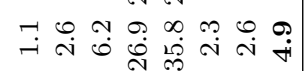 \\
\hline 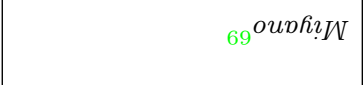 & 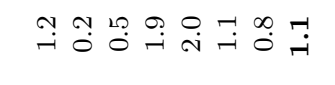 & 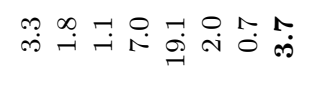 & 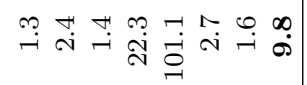 \\
\hline 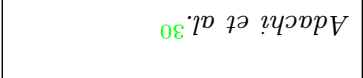 & 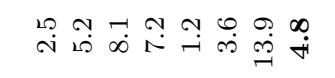 & 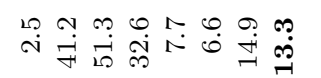 & 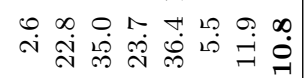 \\
\hline 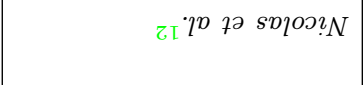 & 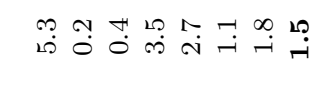 & 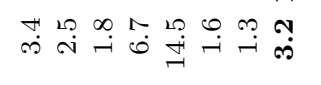 & 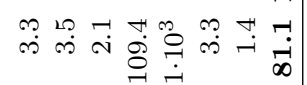 \\
\hline 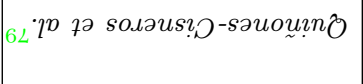 & 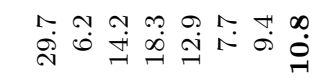 & 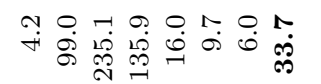 & 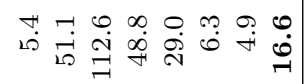 \\
\hline 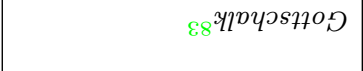 & 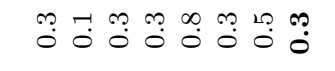 & 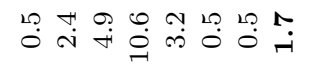 & 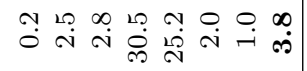 \\
\hline 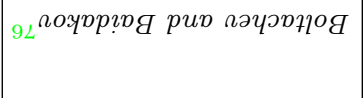 & 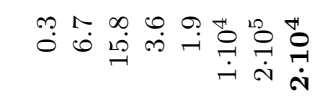 & 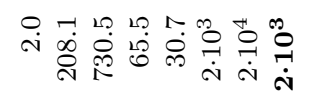 & 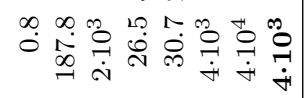 \\
\hline$\varsigma_{L} s s ə H$ & 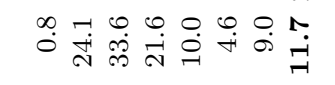 & 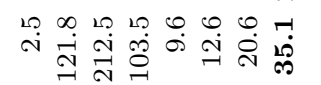 & 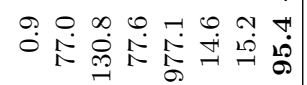 \\
\hline 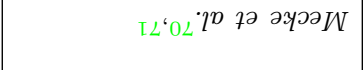 & 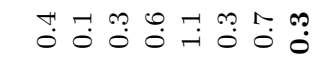 & 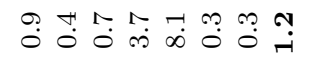 & 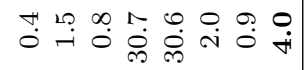 \\
\hline ¿я & 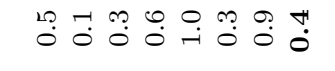 & 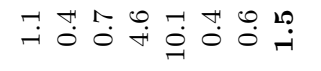 & 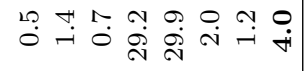 \\
\hline${ }_{89} l^{D}$ วə sp.lqnoY & 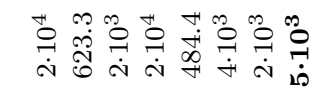 & 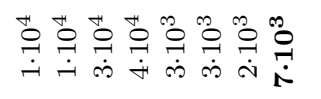 & 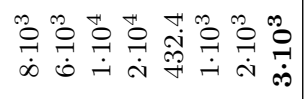 \\
\hline y.10m s? & 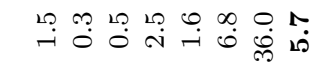 & 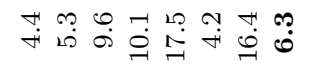 & 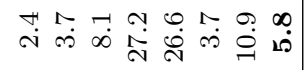 \\
\hline 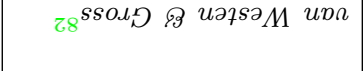 & 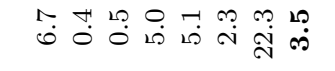 & 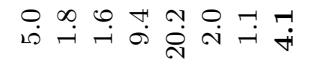 & 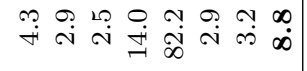 \\
\hline 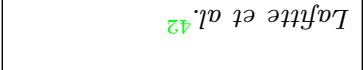 & 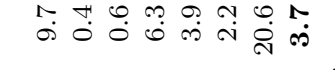 & 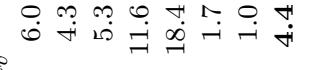 & 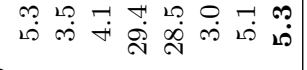 \\
\hline 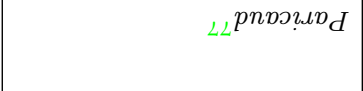 & 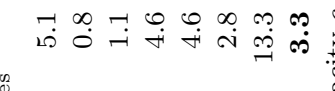 & 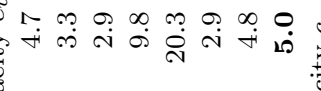 & 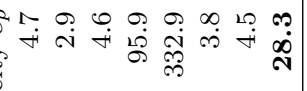 \\
\hline 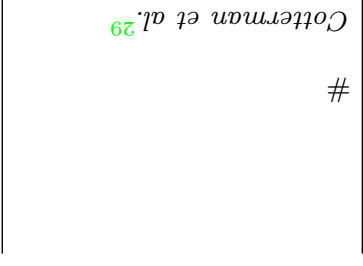 & 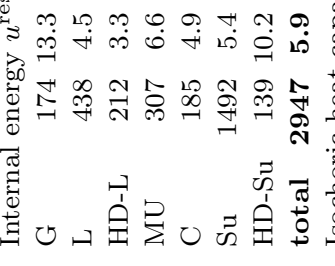 & 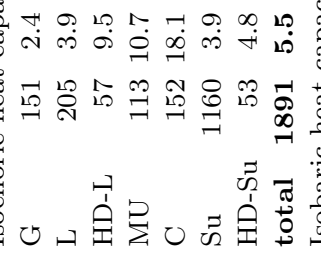 & 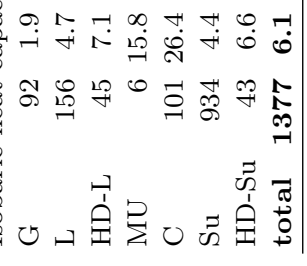 \\
\hline
\end{tabular}




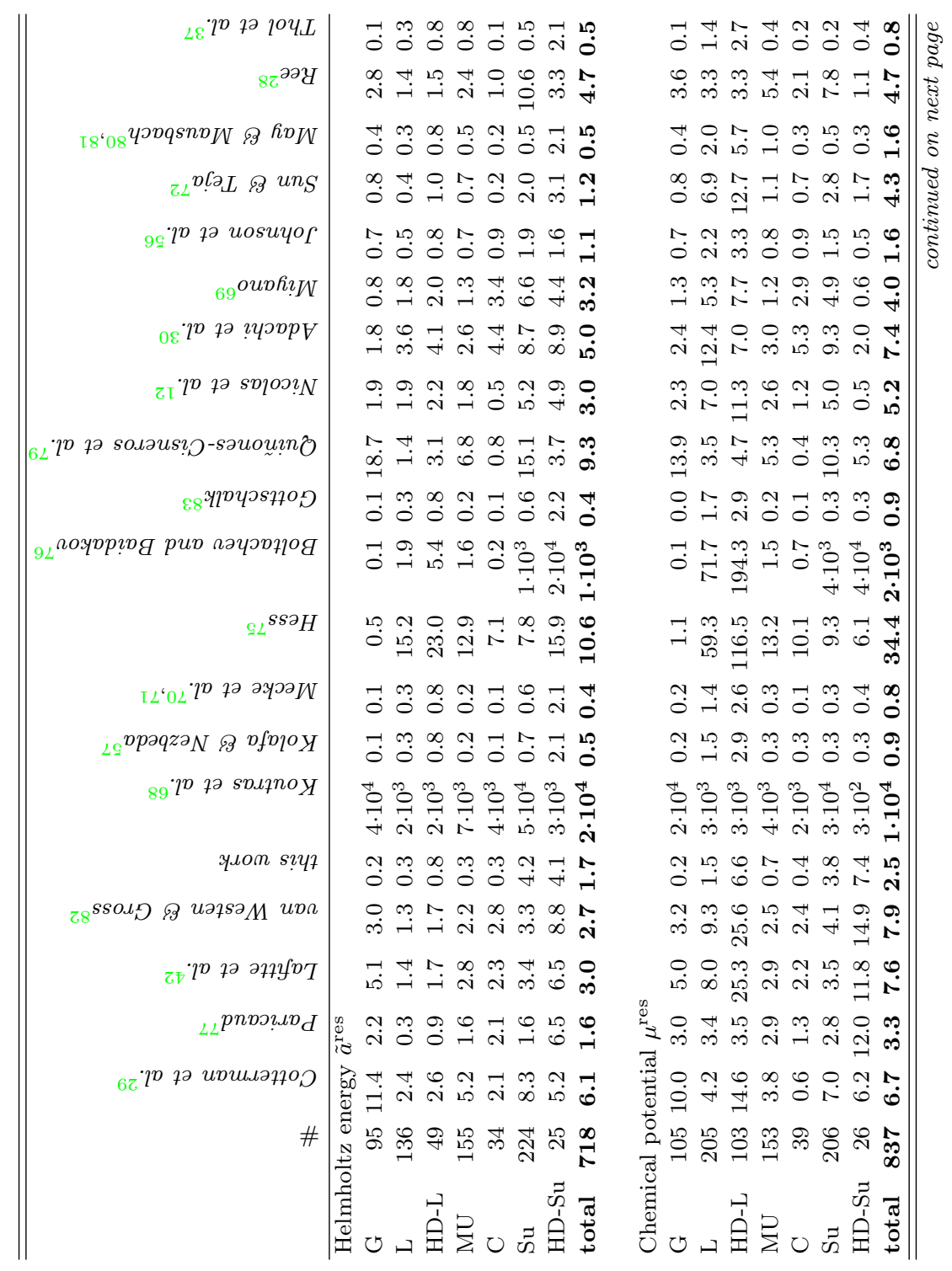




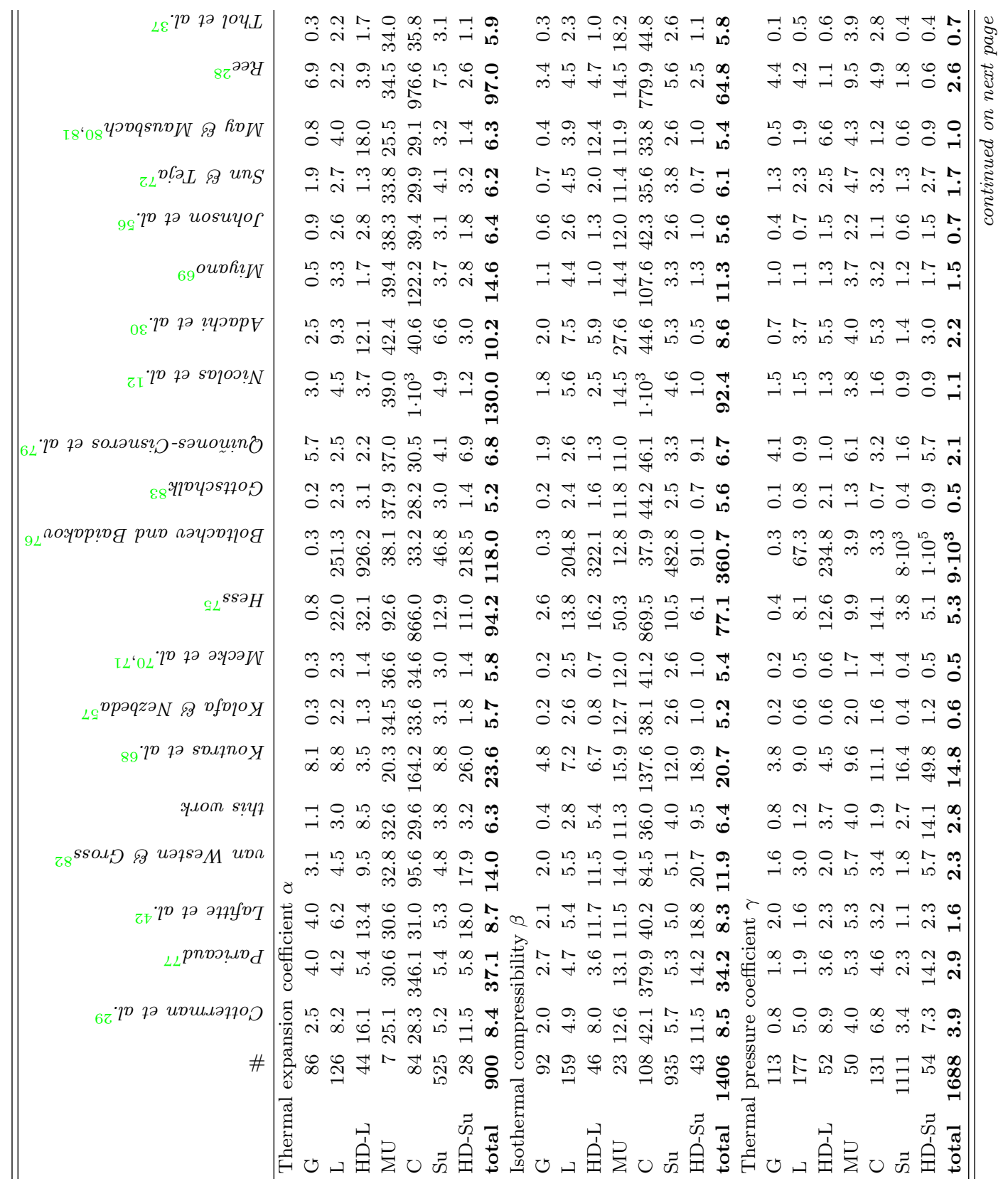




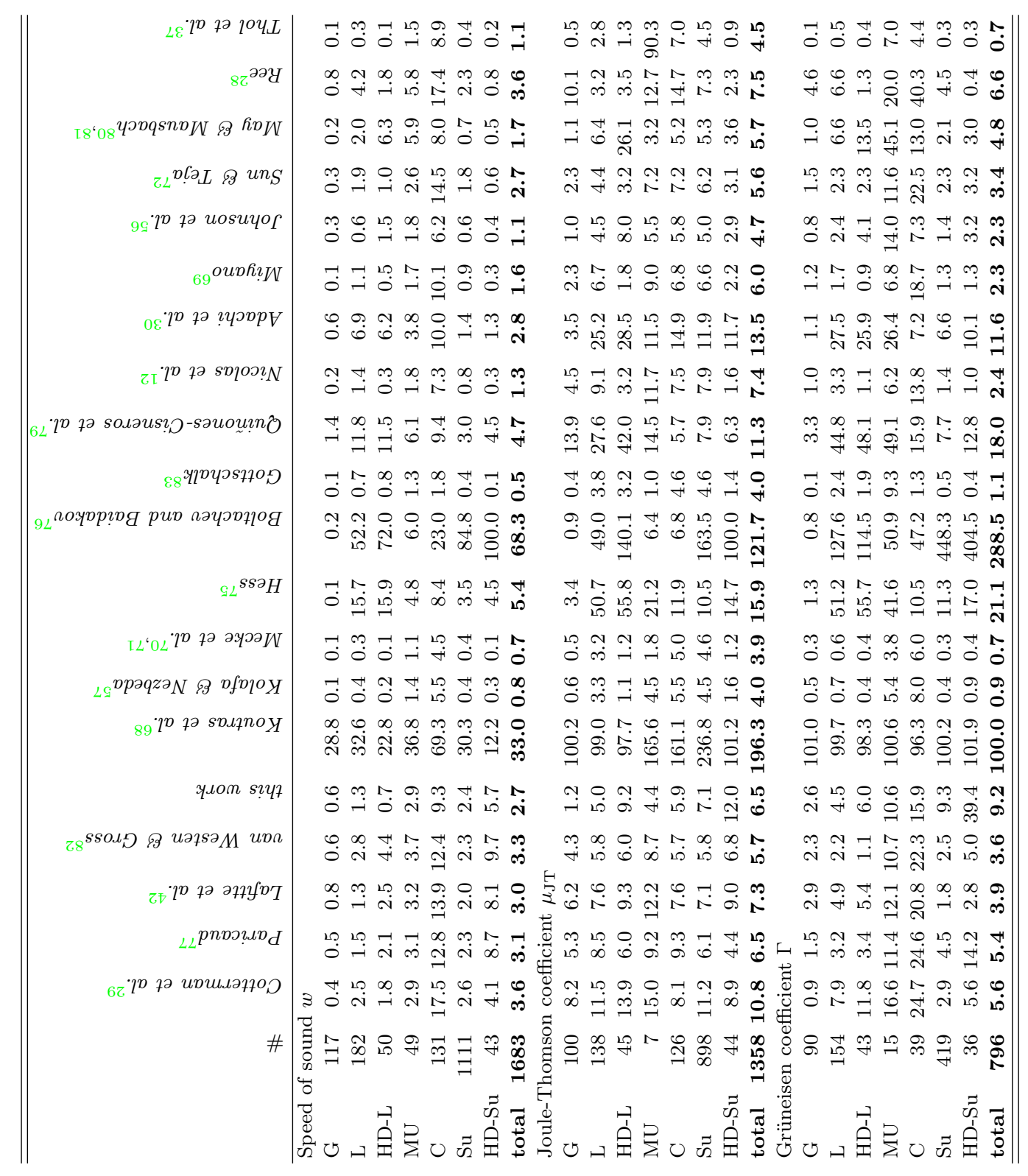


For all considered LJ EOS, the thermodynamic properties were computed from their relations to the reduced Helmholtz energy $\tilde{a}=a / T$ and its derivatives with respect to the density and the inverse temperature using the following notation:

$$
\tilde{a}_{n m}=\tilde{a}_{n m}^{\mathrm{id}}+\tilde{a}_{n m}^{\mathrm{res}}=(1 / T)^{n} \rho^{m} \frac{\partial^{n+m}\left(\tilde{a}^{\mathrm{id}}+\tilde{a}^{\mathrm{res}}\right)}{\partial(1 / T)^{n} \partial \rho^{m}}
$$

where 'id' and 'res' indicate the ideal gas contribution and the residual contribution to the Helmholtz energy, respectively. Only, data for $n, m=0,1,2$ were considered here. This formalism was also applied to pressure-explicit LJ EOS, cf. Table I. As the Lennard-Jones fluid is a monoatomic gas, its ideal gas heat capacity is a priori known. The following thermodynamic properties were studied here ${ }^{32,37}$ :

thermal properties

pressure

$$
p=-\left(\frac{\partial a}{\partial v}\right)_{T}=\rho T\left(1+\tilde{a}_{01}^{\mathrm{res}}\right)
$$

caloric properties

residual internal energy

$$
u^{\mathrm{res}}=a^{\mathrm{res}}+T s^{\mathrm{res}}=T \tilde{a}_{10}^{\mathrm{res}}
$$

isobaric heat capacity

$$
c_{p}=\left(\frac{\partial h}{\partial T}\right)_{p}=-\left(\tilde{a}_{20}^{\mathrm{id}}+\tilde{a}_{20}^{\mathrm{res}}\right)+\frac{\left(1+\tilde{a}_{01}^{\mathrm{res}}-\tilde{a}_{11}^{\mathrm{res}}\right)^{2}}{1+2 \tilde{a}_{01}^{\mathrm{res}}+\tilde{a}_{02}^{\mathrm{res}}}
$$

isochoric heat capacity

$$
c_{v}=\left(\frac{\partial u}{\partial T}\right)_{\rho}=-\left(\tilde{a}_{20}^{\mathrm{id}}+\tilde{a}_{20}^{\mathrm{res}}\right)
$$

entropic properties

residual chemical potential

$$
\mu^{\mathrm{res}}=h^{\mathrm{res}}-T s^{\mathrm{res}}=T\left(1+\tilde{a}_{00}^{\mathrm{res}}+\tilde{a}_{01}^{\mathrm{res}}\right)
$$

$2^{\text {nd }}$-order thermodynamic derivatives

thermal pressure coefficient

$$
\gamma=\left(\frac{\partial p}{\partial T}\right)_{\rho}=\rho\left(1+\tilde{a}_{01}^{\mathrm{res}}-\tilde{a}_{11}^{\mathrm{res}}\right)
$$

isothermal compressibility

$$
\beta=\left(\rho \frac{\partial p}{\partial \rho}\right)_{T}^{-1}=\frac{1}{\rho T\left(1+2 \tilde{a}_{01}^{\mathrm{res}}+\tilde{a}_{02}^{\mathrm{res}}\right)}
$$


thermal expansion coefficient $\quad \alpha=\beta \gamma=\frac{\left(\frac{\partial p}{\partial T}\right)_{\rho}}{\rho\left(\frac{\partial p}{\partial \rho}\right)_{T}}=\frac{1+\tilde{a}_{01}^{\mathrm{res}}-\tilde{a}_{11}^{\mathrm{res}}}{T\left(1+2 \tilde{a}_{01}^{\mathrm{res}}+\tilde{a}_{02}^{\mathrm{res}}\right)}$

speed of sound

$$
w=\sqrt{\left(\frac{\partial p}{\partial \rho}\right)_{s}}=\left(T\left(\left(1+2 \tilde{a}_{01}^{\mathrm{res}}+\tilde{a}_{02}^{\mathrm{res}}\right)-\frac{\left(1+\tilde{a}_{01}^{\mathrm{res}}-\tilde{a}_{11}^{\mathrm{res}}\right)^{2}}{\tilde{a}_{20}^{\mathrm{id}}+\tilde{a}_{20}^{\mathrm{res}}}\right)\right)^{0.5}
$$

Joule-Thomson coefficient

$$
\begin{aligned}
& \mu_{\mathrm{JT}}=\left(\frac{\partial T}{\partial p}\right)_{h} \\
& =\rho^{-1} \frac{-\left(\tilde{a}_{01}^{\mathrm{res}}+\tilde{a}_{02}^{\mathrm{res}}+\tilde{a}_{11}^{\mathrm{res}}\right)}{\left(1+\tilde{a}_{01}^{\mathrm{res}}-\tilde{a}_{11}^{\mathrm{res}}\right)^{2}-\left(\tilde{a}_{20}^{\mathrm{id}}+\tilde{a}_{20}^{\mathrm{res}}\right)\left(1+2 \tilde{a}_{01}^{\mathrm{res}}+\tilde{a}_{02}^{\mathrm{res}}\right)} \\
& \Gamma=\frac{\left(\frac{\partial p}{\partial T}\right)_{\rho}}{\rho c_{v}}=\frac{1+\tilde{a}_{01}^{\mathrm{res}}-\tilde{a}_{11}^{\mathrm{res}}}{-\tilde{a}_{20}^{\mathrm{id}}-\tilde{a}_{20}^{\mathrm{res}}}
\end{aligned}
$$

\section{Thermal Properties}

The $\mathrm{AAD}_{i j k}$ computed according to Eq. (3) for the pressure data $j=p$ is given in Table IV for all considered LJ EOS $i$ and all regions $k$, except for the extreme temperature region $k=$ Ex-T, for which the corresponding results are presented in Table V. Table IV furthermore reports the absolute average deviations from the (exact) values for the second and third virial coefficient $k=B, C$, which were taken from the literature ${ }^{11}$ (Refs. $^{12,72,139,160-162}$ for second virial coefficient data and Refs. ${ }^{72,139,160-163}$ for third virial coefficient data).

The overall best description of the $p v T$ data is found for the LJ EOS of Gottschalk ${ }^{83}$, Thol et $a l^{37}$, Mecke et al. ${ }^{70,71}$, and Kolafa and Nezbeda ${ }^{57}$. However, the LJ EOS of Thol et al. ${ }^{37}$ and Gottschalk ${ }^{83}$ exhibit extreme deviations in the metastable/ unstable region (see above). The LJ EOS from the present work describes the $p v T$ data in the gas, liquid, and critical region as good as the best LJ EOS in those regions. In the HD liquid, and metastable/ unstable region, the LJ EOS from the present work has slightly larger mean deviations than the best LJ EOS in those regions.

Also the LJ EOS of Johnson et al. ${ }^{56}$ gives a good description of the pvT data (cf. Table IV); however, it is somewhat less precise than the three aforementioned LJ EOS. The LJ 
EOS of Sun and Teja ${ }^{72}$, May and Mausbach ${ }^{80,81}$, Quiñones-Cisneros et al. ${ }^{79}$, and Miyano ${ }^{69}$ are in turn slightly less precise than the LJ EOS of Johnson et al. ${ }^{56}$. The theoretically-based LJ EOS of Cotterman et al. ${ }^{29}$, Lafitte et al. ${ }^{42}$, Paricaud ${ }^{77}$, and van Westen and Gross ${ }^{82}$ overall yield an absolute average deviation two to three times larger than the most precise LJ EOS, cf. Table IV. In general no fluid region is represented significantly better or worse than the others by the theoretically-based LJ EOS. The performance of the LJ EOS from Cotterman et al. ${ }^{29}$, Lafitte et al. ${ }^{42}$, Paricaud ${ }^{77}$, and van Westen and Gross $^{82}$ regarding the $p v T$ behavior is overall similar. Also the LJ EOS of Nicolas et al. ${ }^{12}$ has a similar performance regarding the $p v T$ data as the theoretically-based LJ EOS from Refs. ${ }^{29,42,77,82}$, which is surprising considering the fact that the LJ EOS of Nicolas et al. ${ }^{12}$ is one of the oldest LJ EOS considered here. The LJ EOS from the present work performs significantly better than the other theoretically-based LJ EOS, which is likely due to the parametrization procedure (see Supplementary Material). The LJ EOS of Koutras et al. ${ }^{68}$, Hess $^{75}$, Ree ${ }^{28}$, Boltachev and Baidakov ${ }^{76}$, and Adachi et al. ${ }^{30}$ exhibit large deviations in all fluid regions $\left(\mathrm{AAD}_{i, j=p}>10 \%\right)$.

The isotherms obtained from the LJ EOS of Boltachev and Baidakov ${ }^{76}$ exhibit an artificial maximum in the supercritical region, which results in gross deviations in the HD liquid, supercritical and HD supercritical region. This also holds for properties discussed in the following. The LJ EOS of Boltachev and Baidakov ${ }^{76}$ is therefore discarded from the following discussions.

For the second and third virial coefficients $B$ and $C$, the obtained absolute average deviations are reported in the bottom section of Table IV. The second virial coefficient $B$ is exactly described by the LJ EOS of Gottschalk ${ }^{83}$ and Hess ${ }^{75}$, which comprise the corresponding expression in their mathematical form. Good results are also obtained for the LJ EOS of Paricaud ${ }^{77}$, Kolafa and Nezbeda ${ }^{57}$, Mecke et al. ${ }^{70,71}$, Boltachev and Baidakov ${ }^{76}$, Miyano ${ }^{69}$, and Thol et al. ${ }^{37}$. The third virial coefficient $C$ is described best by the LJ EOS of Thol et al. ${ }^{37}$. Also the LJ EOS of Gottschalk ${ }^{83}$ yields an excellent description of $C$ in a wide temperature range, but a wrong limit at low temperatures yields an overall large absolute average deviation. A detailed discussion on the performance of LJ EOS on the virial coefficients and their relation to the characteristic curves can be found in a follow-up work $^{164}$. 


\section{Caloric Properties}

Also the absolute average deviations $\mathrm{AAD}_{i j k}$ for the residual internal energy $j=u^{\mathrm{res}}$ (for brevity, the addition 'residual' is omitted in the following) in the different fluid regions $k$ are reported in Table IV for all studied LJ EOS $i$. As for the $p v T$ data, the LJ EOS of Mecke et al. ${ }^{70,71}$, Gottschalk ${ }^{83}$, Thol et al..$^{37}$, and Kolafa and Nezbeda ${ }^{57}$ give the best descriptions of the internal energy data. The LJ EOS from the present work performs well in most fluid regions, i.e. only slightly exceeds the absolute average deviations from the best LJ EOS, but becomes unreliable in the supercritical and HD supercritical region. This is likely due to the simplifications of the temperature-dependent diameter, cf. Eq. (24), and hence an inadequate description of the soft repulsive interactions ${ }^{165}$ of the PC-SAFT monomer equation in that fluid region (details are given in the Supplementary Material).

The LJ EOS of Johnson et al. ${ }^{56}$, May and Mausbach ${ }^{80,81}$, Nicolas et al. ${ }^{12}$, Sun and Teja ${ }^{72}$, and Miyano $^{69}$ exhibit total deviations $\mathrm{AAD}_{i, j=u^{\text {res }}}$ for the internal energy of about $1 \ldots 2 \%$, cf. Table IV, and are thereby less precise than the aforementioned LJ EOS ${ }^{37,57,70,71,83}$ - in agreement with what was observed for the $p v T$ data.

The $\mathrm{AAD}_{i, j=u^{\text {res }}}$ obtained for the theoretically-based LJ EOS of Cotterman et al. ${ }^{29}$, Lafitte et al. $^{42}$, Paricaud ${ }^{77}$, van Westen and Gross $^{82}$, and the LJ EOS from the present work is about an order of magnitude larger than for the most precise LJ EOS (Refs. ${ }^{37,57,70,71,83}$ ). The deviations of the theoretically-based LJ EOS are particularly large in the gas and high density supercritical region. Nevertheless, the theoretically-based LJ EOS of Lafitte et al. ${ }^{42}$, Paricaud ${ }^{77}$, van Westen and Gross $^{82}$, and from the present work show a good performance in the liquid and HD liquid region, where the deviations are not significantly higher than those of the empirical LJ EOS, cf. Table IV.

The empirical LJ EOS of $R e e^{28}$ and Adachi et al..$^{30}$ perform not better than the considered theoretically-based LJ EOS for the internal energy. The LJ EOS of Koutras et al. ${ }^{68}$, Hess $^{75}$, Quiñones-Cisneros et al..$^{79}$, and Boltachev and Baidakov ${ }^{76}$ exhibit mean deviations $\mathrm{AAD}_{i, j=u^{\mathrm{res}}}>10 \%$.

Interestingly, most LJ EOS show a better performance for the internal energy $u^{\text {res }}$ than for the pressure $p$, i.e. $\mathrm{AAD}_{i, j=p}>\mathrm{AAD}_{i, j=u^{\text {res }}}$. This does not hold for the LJ EOS from the present work, which is based on the PC-SAFT monomer equation. The mean deviation for the internal energy data $\mathrm{AAD}_{i, j=u^{\text {res }}}$ is more than three times larger than the corresponding value for the $p v T$ data $\mathrm{AAD}_{i, j=p}$, which is likely due to the mathematical form of the under- 
lying model, namely on the chosen simplification for describing the temperature-dependent diameter and the perturbation terms (see Supplementary Material for details). These simplifications have also been attributed to shortcomings of PC-SAFT regarding its description of the soft repulsion, see Boshkova and Deiters ${ }^{165}$. The simplifications in describing the soft repulsion in the PC-SAFT EOS may also explain its poorer performance for caloric properties at supercritical states that was reported in the literature ${ }^{166-169}$.

The absolute average deviations $\mathrm{AAD}_{i j k}$ of the isochoric and isobaric heat capacity $j=c_{v}$ and $j=c_{p}$, respectively, found for the different LJ EOS $i$ in the different fluid regions $k$ are also reported in Table IV. The four LJ EOS of Thol et al. ${ }^{37}$, Gottschalk ${ }^{83}$, Mecke et al. ${ }^{70,71}$, and Kolafa and Nezbeda ${ }^{57}$ give the best description of the computer experiment data for the heat capacities, which is in line with the findings for the internal energy $u^{\text {res }}$ and the $p v T$ data. The LJ EOS of Thol et al. ${ }^{37}$ has large deviations in the critical and metastable/ unstable region, but performs slightly better than the LJ EOS of Mecke et al. ${ }^{70,71}$ and Kolafa and Nezbed $a^{57}$ in the gas and in both the high density liquid and high density supercritical region. While the LJ EOS of Gottschalk ${ }^{83}$ gives a slightly better description of the $p v T$ data than that of Thol et al. ${ }^{37}$, this is reversed for the caloric properties in most fluid regions except the critical and metastable/ unstable region.

For the isochoric and isobaric heat capacity, the LJ EOS of Johnson et al. ${ }^{56}$ and Sun and $T e j a^{72}$ have slightly increased absolute average deviations in most fluid regions compared to the four best LJ EOS. The theoretically-based LJ EOS of Cotterman et al. ${ }^{29}$, Lafitte et al. ${ }^{42}$, Paricaud ${ }^{77}$, van Westen and Gross $^{82}$, and the LJ EOS from the present work are less precise than the best empirical ones for the isochoric and isobaric heat capacity, but the difference is only about a factor of two to four instead an order of magnitude as found for the internal energy data.

The LJ EOS of Nicolas et al. ${ }^{12}$ and Paricaud ${ }^{77}$ exhibit large relative deviations of the isobaric heat capacity data in the critical region, which is due to the significantly overestimated critical temperature of these LJ EOS. The large deviations found for both isochoric and isobaric heat capacity data for the LJ EOS of Thol et al. ${ }^{37}$ in the critical and metastable/ unstable region are probably a result of the multiple van der Waals loops and the crossing of the isotherms in the critical region. However, it should be noted that the critical temperature of the LJ EOS of Thol et al. ${ }^{37}$ is in excellent agreement with the presumed true value (see above). 
The LJ EOS of Koutras et al. ${ }^{68}$, Hess ${ }^{75}$, Ree $^{28}$, Adachi et al. ${ }^{30}$, Boltachev and Baidakov ${ }^{76}$, and Quiñones-Cisneros et al. ${ }^{79}$ exhibit for both $j=c_{v}$ and $j=c_{p}$ mean deviations $\mathrm{AAD}_{i j} \gtrsim$ $10 \%$.

\section{Entropic Properties}

The results obtained for performance of the considered LJ EOS $i$ on entropic properties $j=$ (residual reduced Helmholtz energy $\tilde{a}^{\text {res }}=a^{\text {res }} / T$ and chemical potential $\mu^{\text {res }}-$ mentioning 'residual' is again omitted for brevity in the following) in the different fluid regions $k$ are also given in Table IV. The LJ EOS of Thol et al. ${ }^{37}$, Gottschalk ${ }^{83}$, Mecke et al. ${ }^{70,71}$, and Kolafa and Nezbeda ${ }^{57}$ give a generally similar precise description of the entropic properties, cf. Table IV. The relative deviation of the LJ EOS of Johnson et al. ${ }^{56}$ is approximately twice as large as those of the four best LJ EOS $37,57,70,71,83$ for both the Helmholtz energy and the chemical potential. The absolute average deviation for the entropic properties obtained for the theoretically-based LJ EOS of Cotterman et al. ${ }^{29}$, Lafitte et al. ${ }^{42}$, Paricaud ${ }^{77}$, and van Westen and Gross ${ }^{82}$, and the LJ EOS from the present work significantly exceed those from the most precise LJ EOS. Large deviations for the entropic properties are found for all theoretically-based LJ EOS in the high density supercritical region. Furthermore, high deviations are found for the LJ EOS of Cotterman et al. ${ }^{29}$ and Lafitte et al. ${ }^{42}$ in the gas region for both the Helmholtz energy and the chemical potential. The LJ EOS of Boltachev and Baidakov ${ }^{76}$, Koutras et al. ${ }^{68}$, and Hess $^{75}$ exhibit $\mathrm{AAD}_{i j}>10 \%$ for both considered entropic properties $j=\tilde{a}^{\text {res }}$ and $j=\mu^{\text {res }}$.

Overall, the results for the entropic properties support the findings from the caloric and thermal properties discussed above. This also indicates that the reference data ${ }^{11}$ for these properties have overall a similar quality and outliers were equally well detected.

\section{Second Order Derivatives of the Helmholtz energy}

The Helmholtz energy is considered here as a function of the inverse temperature and the density, cf. Eq. (5); accordingly the second derivatives are $\tilde{a}_{20}, \tilde{a}_{11}$, and $\tilde{a}_{02}$. Six more thermodynamic properties were studied here that contain at least one of these derivatives: the thermal pressure coefficient $\gamma$, the isothermal compressibility $\beta$, the thermal expansion coefficient $\alpha$, the speed of sound $w$, the Joule-Thomson coefficient $\mu_{\mathrm{JT}}$, and the Grüneisen parameter $\Gamma$, cf. Eqs. (11) - (16). The $\mathrm{AAD}_{i j k}$ results for the LJ EOS $i$ for $j=\alpha, \beta, \gamma, w, \mu_{\mathrm{JT}}, \Gamma$ in the different fluid regions $k$ are given in Table IV.

The thermal pressure coefficient $\gamma$, isothermal compressibility $\beta$, and thermal expan- 
sion coefficient $\alpha$ is best and overall equally well described by the LJ EOS of Kolafa and Nezbeda ${ }^{57}$, Mecke et al. ${ }^{70,71}$, Gottschalk ${ }^{83}$, and Thol et al. ${ }^{37}$ - as it was also found for the thermal, caloric, and entropic properties discussed above.

The LJ EOS of Johnson et al. ${ }^{56}$, May and Mausbach ${ }^{80,81}$, and Sun and Teja ${ }^{72}$ exhibit slightly larger mean deviations for $\alpha, \beta$, and $\gamma$ than the aforementioned LJ EOS, cf. Table IV. Also the LJ EOS of Lafitte et al. ${ }^{42}$, Cotterman et al. ${ }^{29}$, Quiñones-Cisneros et al. ${ }^{79}$, and the LJ EOS from the present work give a reasonably precise description of the $\alpha, \beta$, and $\gamma$ computer experiment data. Mean deviations $\mathrm{AAD}_{i j}>10 \%$ for either $j=\alpha, \beta$, or $\gamma$ were obtained for the LJ EOS of Miyano ${ }^{69}$, Adachi et al. ${ }^{30}$, Ree ${ }^{28}$, Nicolas et al. ${ }^{12}$, Hess ${ }^{75}$, Paricaud ${ }^{77}$, van Westen and Gross ${ }^{82}$, Koutras et al. ${ }^{68}$, and Boltachev and Baidakov ${ }^{76}$. For the latter two LJ EOS mean deviations $\mathrm{AAD}_{i j}>10 \%$ are found for all three properties $j=\alpha, \beta$, and $\gamma$, cf. Table IV.

All five theoretically-based LJ EOS (Cotterman et al. ${ }^{29}$, Lafitte et al. ${ }^{42}$, Paricaud ${ }^{77}$, and van Westen and Gross $^{82}$, and the LJ EOS from the present work) exhibit high relative deviations for $\alpha, \beta$, and $\gamma$ in the high density supercritical and high density liquid region compared to other fluid regions. Furthermore, the theoretically-based LJ EOS of Lafitte et $a l .{ }^{42}$ and Paricaud $d^{77}$ are considerably less precise in the gas region for $j=\alpha, \beta$, and $\gamma$ than the other theoretically-based LJ EOS, cf. Table IV.

The LJ EOS of Nicolas et al. ${ }^{12}$, Hess ${ }^{75}$, Ree ${ }^{28}$, and Paricaud ${ }^{77}$ exhibit large relative deviations for the $\alpha$ and $\beta$ computer experiment data in the critical region, similar to the findings for the heat capacity. This is attributed to the significantly overestimated critical temperature by these LJ EOS (see above).

The findings for $j=w, \mu_{\mathrm{JT}}$, and $\Gamma$ are in line with the findings for the properties discussed above: the three LJ EOS of Kolafa and Nezbeda ${ }^{57}$, Mecke et al. ${ }^{70,71}$, Gottschalk ${ }^{83}$, and Thol et al. ${ }^{37}$ give similarly precise descriptions, followed by that of Johnson et al. ${ }^{56}$. The theoretically-based LJ EOS are significantly less precise.

Distinct performance features for $w, \mu_{\mathrm{JT}}$, and $\Gamma$ are: the LJ EOS of Thol et al. ${ }^{37}$ exhibits large deviations in the metastable/ unstable region for the Joule-Thomson coefficient and the LJ EOS from the present work is significantly less precise than other LJ EOS for the Grüneisen parameter in the supercritical and high density supercritical region. The latter is again attributed to the simplified temperature-dependent diameter (see Appendix). 


\section{Extreme Temperature Region}

The performance of the investigated LJ EOS $i$ in the extreme temperature region, i.e. $T>6$, is summarized in Table $\mathrm{V}$ by reporting the corresponding absolute average deviations $\mathrm{AAD}_{i j k}$ with $k=\mathrm{Ex}-\mathrm{T}$ for all considered homogeneous state properties $j$. Since the reference data in the extreme temperature region covers a large range of states $^{11}$, high absolute average deviations obtained for a given LJ EOS are in some cases a result of a poor performance in only a sub-region of states, cf. the electronic Supplementary Material.

The LJ EOS of Quiñones-Cisneros et al. ${ }^{79}$, Boltachev and Baidakov ${ }^{76}$, and Koutras et al. ${ }^{68}$ give an erratic description of the extreme temperature region. The empirical LJ EOS of Thol et $a l^{37}$ and $R e e^{28}$ give an astonishingly accurate description of thermodynamic properties at extreme temperatures considering the fact that they were parametrized exclusively using data at moderate temperatures. On the contrary, the theoretically-based LJ EOS exhibit large deviations for most properties. This is surprising, considering the fact that the studied theoretically-based LJ EOS are based on perturbation theory, which is expected to perform well at high temperatures, where the attractive forces, i.e. the perturbation contribution, plays a minor role. The assumption that theoretically-based EOS are in general expected to show a better performance upon extrapolation than empirical EOS should not be taken for granted.

The LJ EOS of Mecke et al..$^{70,71}$ and Gottschalk ${ }^{83}$, which are generally precise (see above), exhibit large deviations for the pressure and internal energy at extreme temperatures - the LJ EOS of Mecke et al. ${ }^{70,71}$ also for other properties. A detailed examination of the data (see the electronic Supplementary Material) shows that these large deviations mainly stem from the region $T>50$ and $\rho>2$ (for the LJ EOS of Gottschalk ${ }^{83}$, very few reference data points entail these large deviations, which probably lie beyond the freezing line). The LJ EOS of Johnson et al..$^{56}$, Sun and Teja ${ }^{72}$, May and Mausbach ${ }^{80,81}$, Nicolas et al. ${ }^{12}$, Adachi et al. ${ }^{30}$, $\mathrm{Hess}^{75}$, and Kolafa and Nezbeda ${ }^{57}$ give a reasonable description of the extreme temperature region - except the internal energy data in some cases. 


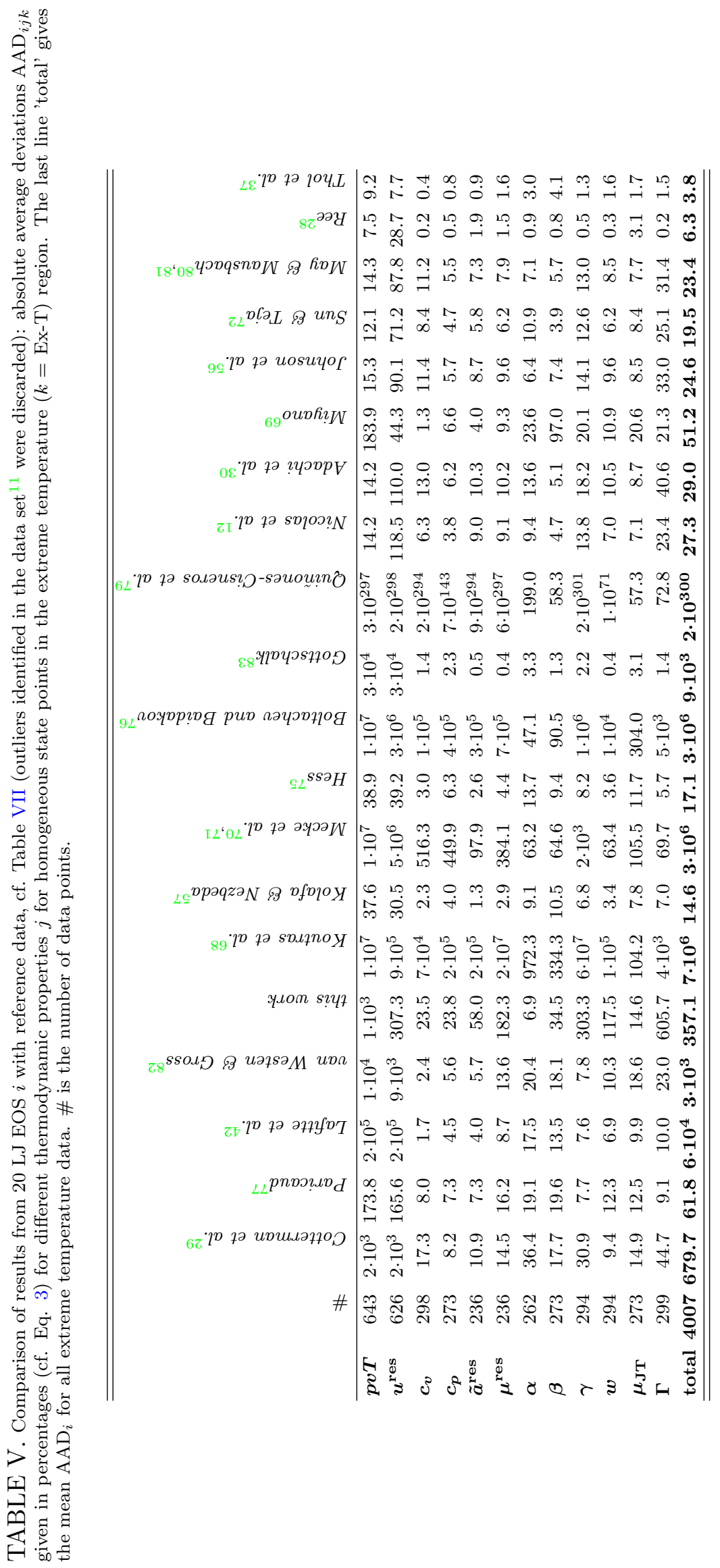




\section{OVERVIEW OF THE PERFORMANCE OF THE LJ EOS}

To enable a systematic comparison of the performance of the considered LJ EOS, we define the performance index $\mathrm{PI}_{i j}$ of a given LJ EOS $i$ for a given property $j$ by setting its absolute average deviation in a given region $k \mathrm{AAD}_{i j k}$ in relation to the lowest absolute average deviation obtained from all considered LJ EOS for that property and region $\mathrm{AAD}_{i=\text { best, } j k}$ and summing up the result over all regions:

$$
\mathrm{PI}_{i j}=1 / N_{\text {reg }} \sum_{k=1}^{N_{\text {reg }}} \frac{\mathrm{AAD}_{i j k}}{\mathrm{AAD}_{i=\text { best }, j k}},
$$

where $N_{\text {reg }}$ indicates the number of fluid regions. Here, $N_{\text {reg }}=7$, as the extreme temperature region was excluded. The $\mathrm{AAD}_{i=\text { best }, j k}$ is the smallest value in a row in Table III and IV for a given property, i.e.

$$
\mathrm{AAD}_{i=\text { best }, j k}=\min _{i}\left(\mathrm{AAD}_{i j k}\right) .
$$

The formalism of Eq. (17) and (18) implies that all fluid regions are equally important and sufficiently densely occupied by reference data points to enable a reasonable evaluation. In fact, out of about 100 considered property-region pairs $j k$, only four have less than 20 data points, cf. Table IV and V. The value $\mathrm{AAD}_{i=\text { best }, j k}$ describes how accurate a given property can be currently described in a given fluid region by the considered LJ EOS.

Low values of the performance index $\mathrm{PI}_{i j}$ are desired. $\mathrm{A} \mathrm{PI}_{i j}=1$ would imply that the LJ EOS $i$ is the best for a given property $j$ in all studied fluid regions. High values of $\mathrm{PI}_{i j}$ imply that the LJ EOS is bad compared to the best competitors. Furthermore, the $\mathrm{AAD}_{i=\text { best, } j k}$ can be considered as an upper limit for the uncertainty, i.e. the scattering of the reference data $Y_{\text {ref }, j k l}$. The ratios of the number of reference data points available for a given property $j$ in the different fluid regions $k$ does not influence the $\mathrm{PI}_{i j}$ value. Eq. (17) was applied to all properties discussed in Table IV $j=\left(p, u^{\text {res }}, c_{v}, c_{p}, \tilde{a}^{\text {res }}, \mu^{\text {res }}, \alpha, \beta, \gamma, w, \mu_{\mathrm{JT}}, \Gamma\right)$ as well as the VLE properties discussed in Table III $j=\left(\rho^{\prime}, \rho^{\prime \prime}, p^{\mathrm{s}}, \Delta h_{\mathrm{v}}\right)$. Since the VLE properties stem all from the same region, no summation has to be carried out in the evaluation of Eq. $(17)$.

Following the differential approach for the extreme temperature region $k=\mathrm{Ex}$-T, i.e. separating the extreme temperature region deviations from the respective $\mathrm{AAD}_{j, \text { total }}$ (cf. Table IV) and reporting the Ex-T data collectively in Table V, this region was not comprised 
in the calculation of the $\mathrm{PI}_{i j}$ values in Eq. (17). Instead, a separate performance index was defined for the comparison of the Ex-T region performance of the LJ EOS on the homogeneous state properties $k$ :

$$
\mathrm{PI}_{i}^{\text {ex- }}=1 / N_{\text {prop }} \sum_{j=1}^{N_{\text {prop }}} \frac{\mathrm{AAD}_{i j, k=\text { ex-T }}}{\mathrm{AAD}_{i=\text { best }, j, k=\text { ex- }}}
$$

where $N_{\text {prop }}=12$ indicates the number of properties $\left(j=p, u^{\text {res }}, c_{v}, c_{p}, \tilde{a}^{\text {res }}, \mu^{\text {res }}\right.$, $\left.\alpha, \beta, \gamma, w, \mu_{\mathrm{JT}}, \Gamma\right)$ for which data are available in that region and the denominator $\mathrm{AAD}_{i=\text { best }, j, k=\text { ex-T }}$ indicates the absolute average deviation of the best performing LJ EOS for a given property $j$ in the region $k=\operatorname{Ex}-\mathrm{T}$ (smallest value in a line in Table $\mathrm{V}$ ).

Table VI summarizes the performance of the investigated LJ EOS regarding the VLE and homogeneous state properties by reporting the $\mathrm{PI}_{i j}$ and $\mathrm{PI}_{i}^{\mathrm{ex}-\mathrm{T}}$ values according Eqs. (17) and (19), respectively. Additionally, it summarizes the maximum number of points with $\mathrm{d} p / \mathrm{d} \rho=0$ and $\mathrm{d}^{2} p / \mathrm{d} \rho^{2}=0$ found in $p-\rho$ isotherms for $0.66<T<1.321$ in the two-phase region (cf. Fig. 5).

The LJ EOS of Kolafa and Nezbeda ${ }^{57}$, Mecke et al. ${ }^{70,71}$, Gottschalk ${ }^{83}$, and Thol et al. ${ }^{37}$ yield an overall similar performance. However, their performance on individual properties differs significantly: the LJ EOS of Mecke et al. ${ }^{70,71}$ and Gottschalk ${ }^{83}$ exhibit large deviations in the extreme-temperature region; the LJ EOS of Thol et al. ${ }^{37}$ and Gottschalk ${ }^{83}$ exhibit multiple van der Waals loops, which yields large $\mathrm{PI}_{i j}$ values for the $p v T$ data. Overall, the LJ EOS of Kolafa and Nezbed $a^{57}$ is found to give the most favorable and robust performance of those four most precise LJ EOS, i.e. for the majority of properties its performance index values are in the range $1.2<\mathrm{PI}_{i j}<1.9$.

The LJ EOS of Johnson et al. ${ }^{56}$ and the LJ EOS from the present work (re-parametrized PC-SAFT monomer) also give a good description of the LJ fluid.

In the group of the MBWR type LJ EOS ${ }^{12,30,56,69,72,80,81}$ (cf. Table I) that of Johnson et $a l{ }^{56}$ yields overall the best performance: comparing the $\mathrm{PI}_{i j}$ values among the MBWR type LJ EOS yields: the LJ EOS of Johnson et al. ${ }^{56}$ has eight lowest performance index values, that of May and Mausbach ${ }^{80,81}$ has four lowest values, and both those of Miyano ${ }^{69}$ and Sun and Teja ${ }^{72}$ have two lowest values.

Comparing the LJ EOS of Johnson et al. ${ }^{56}$ and Kolafa and Nezbeda ${ }^{57}$ (two of the most popular ones; see Supplementary Material), the latter has better performance index values 
for all properties that were considered here. This is surprising, considering the fact that the LJ EOS of Johnson et al. ${ }^{56}$ has approximately four times more citations. This also holds comparing their performance on practically all considered properties in the individual fluid regions, cf. Table III - V.

The theoretically-based LJ EOS yield $\mathrm{PI}_{i j}$ values between about two and ten for the majority of studied properties $j$. Surprisingly, they show a poor performance when used for extrapolations into the extreme temperature region, where they perform worse than some empirical and semi-theoretical LJ EOS. However, the theoretically-based LJ EOS show a good performance in the two-phase region, throughout, i.e. no undulations of the isotherms at most temperatures. Among the studied theoretically-based LJ EOS, the one from the present work shows the best performance as measured by the $\mathrm{PI}_{i j}$ values: for 11 properties it has the best $\mathrm{PI}_{i j}$ value in that group, which is probably due to the parametrization procedure that considers both bulk and VLE properties. However, for the caloric properties, it performs only poorly, which is attributed here to the unfavorable choice of the temperature-dependent diameter term taking a dominant role at high densities and temperatures. This supports the findings of Boshkova and Deiters ${ }^{165}$. The other four theoretically-based LJ EOS (Refs. ${ }^{29,42,77,82}$ ) show overall a similar good performance - with strengths and weaknesses in different properties. 


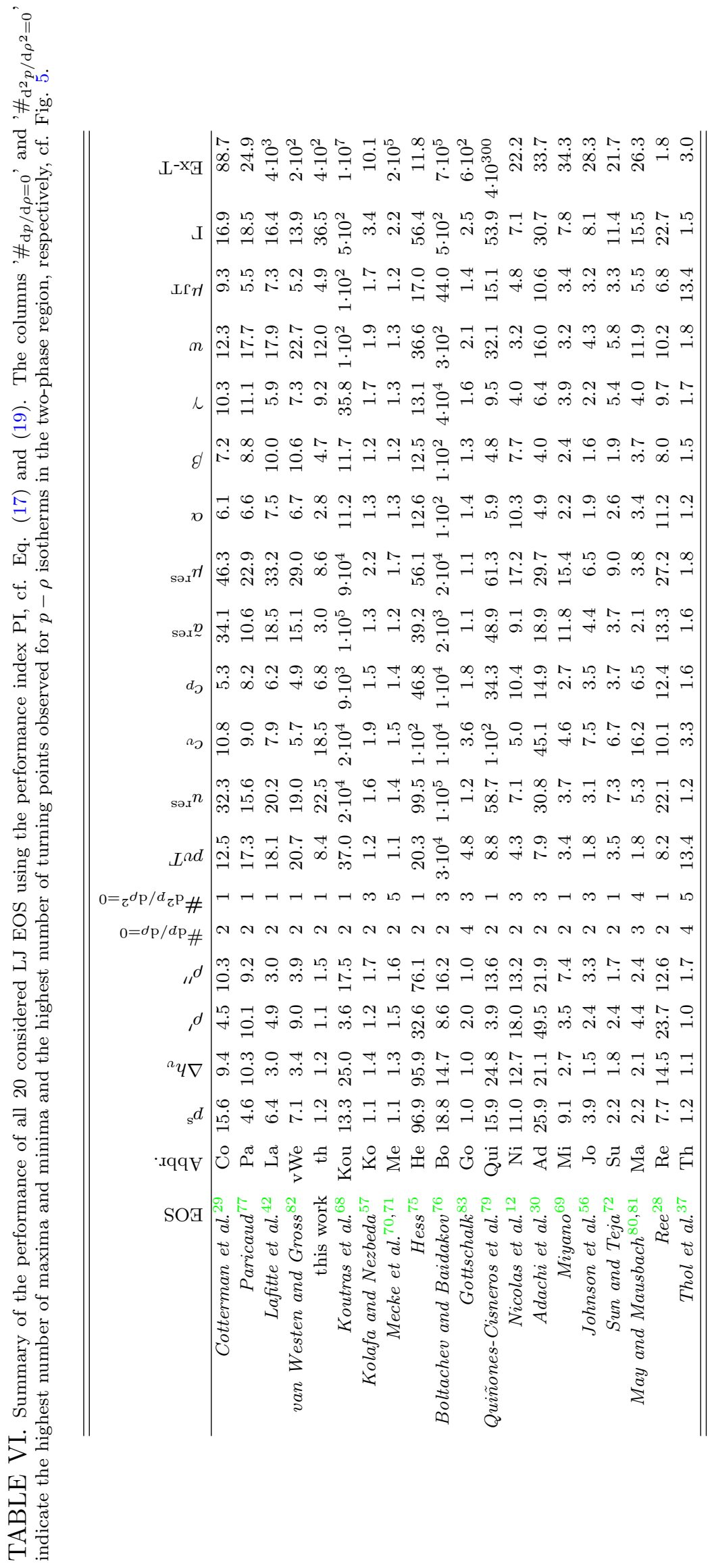




\section{CONCLUSIONS}

In the present work Lennard-Jones equations of state were reviewed and evaluated by comparing their results to an extensive consolidated database of molecular simulation results ${ }^{11}$. Different types of LJ EOS are considered; they are classified here as: empirical EOS, semitheoretical EOS, and theoretically-based EOS. In total, 20 LJ EOS were considered in the present work. The performance of these LJ EOS was investigated and compared for a large number of properties. The numeric values computed from each LJ EOS for each reference data point are reported in the electronic Supplementary Material, which makes the employed test procedure fully transparent and reproducible and enables an extension of the present study to new LJ EOS that may be developed in future work.

Despite the simplicity of the Lennard-Jones fluid and the underlying intermolecular potential and the fact that for more than 50 years LJ EOS have been proposed, no LJ EOS gives a satisfactory description of the LJ fluid, in a sense that it a) represent the available reference data well for most of the properties, i.e. the deviations between the results of the EOS and the computer simulation data are of the same order of magnitude as the uncertainties of the simulation data, b) it shows no unphysical behavior in the two-phase region, and c) it is well-behaved when used for extrapolations to regions in which extreme conditions prevail. Some LJ EOS meet the criterion a) well, namely the LJ EOS of Mecke et al. ${ }^{70,71}$, Gottschalk $^{83}$, and Thol et al. ${ }^{37}$, but they fail to meet the other criteria in one or several ways. Only the LJ EOS of Kolafa and Nezbed $a^{57}$ is found to meet all criteria reasonably well, but is, regarding criterion a) slightly less precise than the three aforementioned LJ EOS. The four LJ EOS of Kolafa and Nezbeda ${ }^{57}$, Mecke et al. ${ }^{70,71}$, Gottschalk ${ }^{83}$, and Thol et $a l .{ }^{37}$ were found to give an overall similarly good description of the Lennard-Jones fluid. However, their performance was found to differ significantly in different fluid regions and thermodynamic properties. The LJ EOS of Kolafa and Nezbeda ${ }^{57}$ is found to be the most robust LJ EOS, cf. Table VI. The LJ EOS of Thol et al. ${ }^{37}$ and Gottschalk ${ }^{83}$ exhibit multiple van der Waals loops and the LJ EOS of Mecke et al. ${ }^{70,71}$ exhibit large deviations at extreme temperature and density.

The LJ EOS of Johnson et al. ${ }^{56}$ has been used extensively in the literature, but is found to be significantly less precise than either of the four aforementioned. Nevertheless, among the MBWR type LJ $\operatorname{EOS}^{12,30,56,69,72,80,81}$, that of Johnson et al. ${ }^{56}$ is found to be the best. 
The four overall most precise LJ EOS $37,57,70,71,83$ are empirical or semi-theoretical LJ EOS; the theoretically-based LJ EOS are found to be overall significantly less precise (for most properties they are about an order of magnitude less precise than the best LJ EOS). Very surprisingly, the theoretically-based LJ EOS are found to give a less precise performance upon extrapolating to extreme temperatures and densities compared to the best empirical LJ EOS. This is particularly striking since the considered LJ EOS are based on Barker and Henderson's perturbation theory, which is expected to perform well at high temperatures, where the attractive forces play a minor role. Hence, the systematic deviations are probably due to the modeling of the soft repulsion in the theory around the hard sphere reference system.

The theoretically-based LJ EOS of Cotterman et al. ${ }^{29}$, Paricaud ${ }^{77}$, Lafitte et al. ${ }^{42}$, and van Westen and Gross $^{82}$ are found to give a good representation of most thermodynamic properties, but are overall significantly less precise than the best empirical and semi-theoretical LJ EOS. Despite the large differences of the year of publication which varies between 1986 and 2017 and differences of the underlying models, the performance of the four theoreticallybased LJ EOS of Refs. ${ }^{29,42,77,82}$ is overall similar.

SAFT type EOS have become very popular in the last 30 years and many variants of these EOS exist. Several of them use monomer terms that were developed to match data of the Lennard-Jones fluid, i.e. the monomer terms are LJ EOS. Four of them were included in the present study ${ }^{12,42,56,57}$. However, the monomer term of PC-SAFT - a particularly important version of SAFT - could not be included directly in the present study, as it was not developed to describe the Lennard-Jones fluid. Therefore, in the present work, the PCSAFT monomer term was re-parameterized using data of the LJ fluid. The resulting LJ EOS is found to be more precise for most properties and fluid regions than the theoretically-based LJ EOS of Refs. ${ }^{29,42,77,82}$, which is probably due to the employed parametrization strategy that takes both VLE and homogeneous data into account. However, the present work reveals also shortcomings of the PC-SAFT monomer term: it exhibits important systematic deviations in the supercritical region - especially for caloric properties. This is attributed to the simplified form of the temperature-dependent diameter, which is used for the modeling of the soft repulsive interactions in the PC-SAFT monomer term.

The comparison and evaluation of LJ EOS employed in the present work is based on a systematic strategy: an extensive and consolidated database is used as reference data that 
comprises a broad variety of properties; each EOS is tested on that reference data; the fluid region is divided into characteristic sub-regions, e.g. in the $T-\rho$ space; each property is studied in each region separately before the results are combined to achieve an overall assessment. This strategy could also be useful for testing other pure component EOS and be applied as a benchmark test for new Lennard-Jones equations of state. 


\section{APPENDIX A - LENNARD-JONES REFERENCE DATABASE}

Table VII gives an overview of the consolidated database of computer experiment data on the LJ fluid used in the present work for the evaluation of the LJ EOS. Table VII also reports the number of data points available in a given reference and the number of outliers identified therein ${ }^{11}$. The latter were discarded in the present work for the evaluation of the LJ EOS. The numeric values computed from each LJ EOS for each reference data point are reported in the electronic Supplementary Material.

TABLE VII: Computer experiment data of the LJ fluid used as reference. The database is adapted from Ref. ${ }^{11}$. The data is sorted for each property chronologically. The column \# reports number of data points and, in brackets, is the number of outliers therein. The column $T$ reports the temperature range in which data are available.

\begin{tabular}{|c|c|c|c|}
\hline Authors & Year & \# & $T$ \\
\hline \multicolumn{4}{|l|}{$\operatorname{VLE} p^{\mathrm{s}}, \rho^{\prime}, \rho^{\prime \prime}, \Delta h_{v}$} \\
\hline Lotfi et al. ${ }^{104}\left(p^{\mathrm{s}}, \rho^{\prime}, \rho^{\prime \prime}, \Delta h_{v}\right)$ & 1992 & $48(4)$ & $0.7-1.5$ \\
\hline Okumura and Yonezawa ${ }^{121}\left(p^{\mathrm{s}}, \rho^{\prime}, \rho^{\prime \prime}\right)$ & 2001 & $15(0)$ & $1.25-1.29$ \\
\hline Errington $^{124}\left(p^{\mathrm{s}}, \rho^{\prime}, \rho^{\prime \prime}\right)$ & 2003 & $9(0)$ & $0.7-1.1$ \\
\hline Errington $^{125}\left(p^{\mathrm{s}}, \rho^{\prime}, \rho^{\prime \prime}\right)$ & 2003 & $36(3)$ & $0.7-1.2$ \\
\hline Mick et al. ${ }^{131}\left(p^{\mathrm{s}}, \rho^{\prime}, \rho^{\prime \prime}, \Delta h_{v}\right)$ & 2013 & $32(8)$ & $0.75-1.3$ \\
\hline Janeček et al. ${ }^{133}\left(p^{\mathrm{s}}, \rho^{\prime}, \rho^{\prime \prime}, \Delta h_{v}\right)$ & 2017 & $24(0)$ & $0.8-1.25$ \\
\hline Stephan et al..$^{11}\left(p^{\mathrm{s}}, \rho^{\prime}, \rho^{\prime \prime}, \Delta h_{v}\right)$ & 2019 & $124(4)$ & $0.69-1.28$ \\
\hline \multicolumn{4}{|l|}{$p v T$} \\
\hline Wood and Parker ${ }^{5}$ & 1957 & $13(8)$ & 2.74 \\
\hline Fickett and Wood $^{181}$ & 1960 & $23(6)$ & $1.92-177.95$ \\
\hline McDonald and Singer ${ }^{182}$ & 1967 & $28(17)$ & $0.72-1.24$ \\
\hline McDonald and Singer ${ }^{9}$ & 1967 & $48(9)$ & $1.45-3.53$ \\
\hline Verlet and Levesque ${ }^{147}$ & 1967 & $7(5)$ & $1.05-2.74$ \\
\hline Verlet $^{8}$ & 1967 & $39(9)$ & $0.59-4.63$ \\
\hline Wood $^{183}$ & 1968 & $41(17)$ & $1.06-100$ \\
\hline Hansen and Verlet ${ }^{10}$ & 1969 & $25(5)$ & $0.75-1.15$ \\
\hline Levesque and Verlet ${ }^{61}$ & 1969 & $25(7)$ & $0.72-3.67$ \\
\hline McDonald and Singer ${ }^{184}$ & 1969 & $28(15)$ & $0.72-1.24$ \\
\hline Hansen $^{185}$ & 1970 & $9(1)$ & $2.74-100$ \\
\hline McDonald and Woodcock ${ }^{186}$ & 1970 & $6(3)$ & $0.75-2.33$ \\
\hline Toxvaerd and Praestgaard 62 & 1970 & $8(2)$ & 1.35 \\
\hline Weeks et al. ${ }^{88}$ & 1971 & $5(1)$ & $0.75-1.35$ \\
\hline McDonald and Singer 63 & 1972 & $56(19)$ & $0.55-1.24$ \\
\hline
\end{tabular}




\begin{tabular}{|c|c|c|}
\hline Authors & Year \# & $T$ \\
\hline Schofield $^{187}$ & $19736(6)$ & $0.73-1.10$ \\
\hline Street et al. ${ }^{188}$ & $197480(45)$ & $0.75-3.05$ \\
\hline Adams $s^{189}$ & $197512(8)$ & $2-4$ \\
\hline Adams $^{97}$ & $197616(7)$ & $1.0-1.2$ \\
\hline Carley $^{190}$ & $197711(3)$ & 1.35 \\
\hline $\operatorname{Adams}^{99}$ & $197931(11)$ & $1.15-1.35$ \\
\hline Nicolas et al. ${ }^{12}$ & $1979108(13)$ & $0.48-6.01$ \\
\hline $\operatorname{Re}^{28}$ & $198011(1)$ & $0.81-2.7$ \\
\hline Yao et al. ${ }^{191}$ & $198212(1)$ & $1.15-1.25$ \\
\hline Powles et al. ${ }^{95}$ & $198237(9)$ & $0.7-1.41$ \\
\hline Lucas 192 & $198610(2)$ & $0.79-1.83$ \\
\hline Shaw ${ }^{193}$ & $1988265(44)$ & $0.6-136.25$ \\
\hline Adachi et al..$^{30}$ & $1988328(250)$ & $0.7-2.95$ \\
\hline Baranyai et al..$^{194}$ & $198912(0)$ & $0.75-1.5$ \\
\hline Saager and Fischer ${ }^{195}$ & $199038(1)$ & $0.57-4$ \\
\hline Sowers and Sandler 67 & $199160(6)$ & $1.35-6$ \\
\hline Lotfi et al. ${ }^{104}$ & $199219(7)$ & $0.7-1.3$ \\
\hline Giaquinta et al. ${ }^{196}$ & $199212(0)$ & $0.75-1.15$ \\
\hline Johnson et al. ${ }^{56}$ & $1993199(6)$ & $0.7-6$ \\
\hline Kolafa et al. ${ }^{197}$ & $199343(2)$ & $0.72-4.85$ \\
\hline Miyano $^{69}$ & $1993112(13)$ & $0.45-100$ \\
\hline Kolafa and Nezbeda ${ }^{57}$ & $199413(0)$ & $0.81-10$ \\
\hline Lustig $^{198}$ & $19942(0)$ & 1.18 \\
\hline Mecke et al. ${ }^{70}$ & $199612(0)$ & $1.32-1.34$ \\
\hline Roccatano et al. ${ }^{199}$ & $199810(3)$ & $1.4-10.0$ \\
\hline Meier 200 & $2002351(4)$ & $0.7-6$ \\
\hline Linhart et al. ${ }^{159}$ & $2005108(18)$ & $0.7-1.2$ \\
\hline Morsali et al. ${ }^{201}$ & $200713(0)$ & 5.01 \\
\hline Baidakov et al. ${ }^{202}$ & $2008208(5)$ & $0.35-2$ \\
\hline Lustig $^{203}$ & $20118(0)$ & $1.02-3.0$ \\
\hline May and Mausbach 204 & $2012205(8)$ & $0.69-6.17$ \\
\hline Yigzawe and Sadus 205,206 & $2012406(24)$ & $1.3-2.62$ \\
\hline Mairhofer and Sadus ${ }^{207}$ & $2013282(8)$ & $1.36-3.05$ \\
\hline Thol et $a l .37$ & $2016197(3)$ & $0.7-9$ \\
\hline Deiters and Neumaier 208 & $2016255(2)$ & $0.67-22.54$ \\
\hline Köster et al. ${ }^{21}$ & $201745(0)$ & $1.01-30.0$ \\
\hline Köster et al. ${ }^{21}$ & $201765(2)$ & $1.01-30.0$ \\
\hline Ustinov $^{209}$ & $2017232(18)$ & $0.76-1.14$ \\
\hline Schultz and Kofke 210 & $2018404(93)$ & $0.68-2272.73$ \\
\hline Stephan et al. ${ }^{11}$ & $2019655(8)$ & $0.7-90$ \\
\hline \multicolumn{3}{|l|}{ Internal energy $u^{\text {res }}$} \\
\hline Wood and Parker ${ }^{5}$ & $1957 \quad 13(9)$ & 2.74 \\
\hline McDonald and Singer 182 & $196727(19)$ & $0.72-1.24$ \\
\hline McDonald and Singer 9 & $196748(44)$ & $1.45-3.53$ \\
\hline Verlet $^{8}$ & $196739(11)$ & $0.59-4.63$ \\
\hline Verlet and Levesque ${ }^{147}$ & $19677(5)$ & $1.05-2.74$ \\
\hline Wood $^{183}$ & $196841(15)$ & $1.06-100$ \\
\hline
\end{tabular}

Continued on next page 


\begin{tabular}{|c|c|c|c|}
\hline Authors & Year & $\#$ & $T$ \\
\hline${\text { Levesque and } \text { Verlet }^{61}}^{1}$ & 1969 & $25(10)$ & $0.72-3.67$ \\
\hline McDonald and Singer ${ }^{184}$ & 1969 & $28(8)$ & $0.72-1.24$ \\
\hline Hansen $^{185}$ & 1970 & $9(5)$ & $2.74-100$ \\
\hline McDonald and Woodcock ${ }^{186}$ & 1970 & $6(2)$ & $0.75-2.33$ \\
\hline Weeks et al. ${ }^{211}$ & 1971 & $5(2)$ & $0.75-1.35$ \\
\hline McDonald and Singer 63 & 1972 & $56(19)$ & $0.55-1.24$ \\
\hline Street et al. ${ }^{188}$ & 1974 & $80(48)$ & $0.75-3.05$ \\
\hline Adams ${ }^{189}$ & 1975 & $12(11)$ & $2-4$ \\
\hline Adams $^{97}$ & 1976 & $16(16)$ & $1.0-1.2$ \\
\hline Torrie and Valleau ${ }^{212}$ & 1977 & $7(6)$ & $0.092-1.35$ \\
\hline Adams ${ }^{99}$ & 1979 & $31(1)$ & $1.15-1.35$ \\
\hline Nicolas et al. ${ }^{12}$ & 1979 & $108(24)$ & $0.48-6.01$ \\
\hline$R e e^{28}$ & 1980 & $11(4)$ & $0.81-2.7$ \\
\hline Yao et al..$^{191}$ & 1982 & $12(7)$ & $1.15-1.25$ \\
\hline Lucas 192 & 1986 & $10(4)$ & $0.79-1.83$ \\
\hline Shaw ${ }^{193}$ & 1988 & $265(68)$ & $0.59-136.25$ \\
\hline Baranyai et al. ${ }^{194}$ & 1989 & $18(0)$ & $0.75-1.5$ \\
\hline Saager and Fischer ${ }^{195}$ & 1990 & $38(0)$ & $0.57-4$ \\
\hline Sowers and Sandler 67 & 1991 & $54(13)$ & $1.35-6$ \\
\hline Lotfi et al. ${ }^{104}$ & 1992 & $19(1)$ & $0.7-1.3$ \\
\hline Giaquinta et al. ${ }^{196}$ & 1992 & $12(0)$ & $0.75-1.15$ \\
\hline Johnson et al..$^{56}$ & 1993 & $199(10)$ & $0.7-6$ \\
\hline Kolafa et al. ${ }^{197}$ & 1993 & $43(1)$ & $0.72-4.85$ \\
\hline Miyano $^{69}$ & 1993 & $112(25)$ & $0.45-100$ \\
\hline Kolafa and Nezbeda ${ }^{57}$ & 1994 & $13(1)$ & $0.81-10$ \\
\hline Lustig $^{198}$ & 1994 & $2(0)$ & 1.18 \\
\hline Mecke et al. ${ }^{70}$ & 1996 & $12(0)$ & $1.32-1.34$ \\
\hline Roccatano et al. ${ }^{199}$ & 1998 & $10(6)$ & $1.4-10.0$ \\
\hline Meier ${ }^{200}$ & 2002 & $351(7)$ & $0.7-6$ \\
\hline Baidakov et al. ${ }^{202}$ & 2008 & 201(9) & $0.35-2$ \\
\hline May and Mausbach 204 & 2012 & $218(7)$ & $0.68-6.17$ \\
\hline Yigzawe and Sadus 205,206 & 2012 & $346(96)$ & $1.31-2.62$ \\
\hline Mairhofer and Sadus 207 & 2013 & $282(0)$ & $1.36-3.05$ \\
\hline Thol et al. ${ }^{37}$ & 2016 & $197(3)$ & $0.7-9$ \\
\hline Deiters and Neumaier 208 & 2016 & $255(2)$ & $0.67-22.54$ \\
\hline Köster et al..$^{21}$ & 2017 & $45(0)$ & $1.01-30.0$ \\
\hline Köster et al. ${ }^{21}$ & 2017 & $65(11)$ & $1.01-30.0$ \\
\hline Ustinov $^{209}$ & 2017 & $232(105)$ & $0.76-1.14$ \\
\hline Schultz and Kofke 210 & 2018 & $404(98)$ & $0.68-2272.73$ \\
\hline Stephan et al. ${ }^{11}$ & 2019 & $655(14)$ & $0.7-90$ \\
\hline \multicolumn{4}{|l|}{ Isochoric heat capacity $c_{v}$} \\
\hline Wood and Parker & 1957 & $11(6)$ & 2.74 \\
\hline McDonald and Singer 182 & 1967 & $26(16)$ & $0.72-1.24$ \\
\hline McDonald and Singer 9 & 1967 & $48(29)$ & $1.45-3.53$ \\
\hline Adams $^{189}$ & 1975 & $12(5)$ & $2-4$ \\
\hline Adams $^{99}$ & 1979 & $31(12)$ & $1.15-1.35$ \\
\hline Saager et al. ${ }^{213}$ & 1990 & $12(4)$ & $1.1-1.35$ \\
\hline
\end{tabular}

Continued on next page 


\begin{tabular}{|c|c|c|c|}
\hline Authors & Year & \# & $\bar{T}$ \\
\hline Boda et $a ._{.}^{214}$ & 1996 & $9(0)$ & $1.31-2$ \\
\hline Roccatano et al. ${ }^{199}$ & 1998 & $10(2)$ & $1.4-10.0$ \\
\hline Meier $^{200}$ & 2002 & $327(12)$ & $0.7-6$ \\
\hline Baidakov et al. ${ }^{202}$ & 2008 & $208(11)$ & $0.35-2$ \\
\hline May and Mausbach ${ }^{204}$ & 2012 & $218(11)$ & $0.68-6.17$ \\
\hline Yigzawe and Sadus 205,206 & 2012 & $406(32)$ & $1.3-2.62$ \\
\hline Mairhofer and Sadus ${ }^{207}$ & 2013 & $282(3)$ & $1.36-3.05$ \\
\hline Thol et al. ${ }^{37}$ & 2016 & $197(5)$ & $0.7-9$ \\
\hline Köster et al. ${ }^{21}$ & 2017 & $45(0)$ & $1.01-30.0$ \\
\hline Köster et al. ${ }^{21}$ & 2017 & $65(2)$ & $1.01-30.0$ \\
\hline Stephan et al. ${ }^{11}$ & 2019 & $515(4)$ & $0.7-90$ \\
\hline \multicolumn{4}{|l|}{ Isobaric heat capacity $c_{p}$} \\
\hline Boda et al. ${ }^{214}$ & 1996 & $41(6)$ & $0.65-1.9$ \\
\hline Lustig $^{203}$ & 2011 & $6(0)$ & $1.02-3.0$ \\
\hline May and Mausbach ${ }^{204}$ & 2012 & $202(4)$ & $0.69-6.17$ \\
\hline Yigzawe and Sadus ${ }^{205,206}$ & 2012 & $406(52)$ & $1.3-2.62$ \\
\hline Mairhofer and Sadus 207 & 2013 & $282(7)$ & $1.36-3.05$ \\
\hline Thol et al. ${ }^{37}$ & 2016 & $197(12)$ & $0.7-9$ \\
\hline Köster et al. ${ }^{21}$ & 2017 & $45(0)$ & $1.01-30.0$ \\
\hline Köster et al. ${ }^{21}$ & 2017 & $65(2)$ & $1.01-30.0$ \\
\hline Stephan et al. ${ }^{11}$ & 2019 & $515(8)$ & $0.7-90$ \\
\hline \multicolumn{4}{|l|}{ Helmholtz energy $\tilde{a}^{\text {res }}$} \\
\hline Levesque and Verlet ${ }^{61}$ & 1969 & $8(4)$ & 1.35 \\
\hline Hansen and Verlet ${ }^{10}$ & 1969 & $25(13)$ & $0.75-1.15$ \\
\hline Weeks et al. ${ }^{88}$ & 1971 & $5(0)$ & $0.75-1.35$ \\
\hline Weeks et al. ${ }^{211}$ & 1971 & $27(14)$ & $0.75-1.35$ \\
\hline Torrie and Valleau ${ }^{212}$ & 1977 & $16(6)$ & $0.75-2.74$ \\
\hline Baranyai and Evans ${ }^{194}$ & 1989 & $6(0)$ & 1.15 \\
\hline Cuadros et al. ${ }^{73}$ & 1996 & $269(268)$ & $0.7-2.6$ \\
\hline Hong and Jhon ${ }^{215}$ & 1997 & $36(22)$ & $0.59-2.89$ \\
\hline Hong and Jang 216 & 2003 & $22(11)$ & $0.59-2.85$ \\
\hline Thol et al. ${ }^{37}$ & 2016 & $197(10)$ & $0.7-9$ \\
\hline Köster et al. ${ }^{21}$ & 2017 & $65(3)$ & $1.3-30.0$ \\
\hline Stephan et al. ${ }^{11}$ & 2019 & $655(18)$ & $0.7-90$ \\
\hline \multicolumn{4}{|l|}{ Chemical potential $\mu^{\text {res }}$} \\
\hline Adams ${ }^{189}$ & 1975 & $12(11)$ & $2-4$ \\
\hline$A_{d a m s} 97$ & 1976 & $16(7)$ & $1-1.2$ \\
\hline Adams $s^{99}$ & 1979 & $31(5)$ & $1.15-1.35$ \\
\hline Yao et al. ${ }^{191}$ & 1982 & $12(0)$ & $1.15-1.25$ \\
\hline Powles et al. ${ }^{95}$ & 1982 & $37(35)$ & $0.7-1.41$ \\
\hline Lotfi et al. ${ }^{104}$ & 1992 & $19(1)$ & $0.7-1.3$ \\
\hline $\operatorname{Han}^{217}$ & 1992 & $3(1)$ & 1.2 \\
\hline Kolafa et al. ${ }^{197}$ & 1993 & $7(1)$ & $1.2-1.45$ \\
\hline Lustig $^{198}$ & 1994 & $2(2)$ & 1.18 \\
\hline Thol et al. ${ }^{37}$ & 2016 & $197(7)$ & $0.7-9$ \\
\hline
\end{tabular}

Continued on next page 


\begin{tabular}{llll}
\hline \hline Authors & Year $\#$ & T \\
\hline Köster et al. $^{21}$ & 2017 & $65(0)$ & $1.3-30.0$ \\
Ustinov $^{209}$ & 2017 & $232(57)$ & $0.76-1.14$ \\
Stephan et al. $^{11}$ & 2019 & $656(20)$ & $0.7-90$
\end{tabular}

Thermal expansion coefficient $\alpha$

McDonald and Singer ${ }^{182}$

Adams ${ }^{189}$

Yigzawe and Sadus 205,206

Thol et al. ${ }^{37}$

Köster et al. ${ }^{21}$

Köster et al. ${ }^{21}$

Stephan et al. ${ }^{11}$

Isothermal compressibility $\beta$

McDonald and Singer ${ }^{182}$

Adams ${ }^{189}$

Adams $^{99}$

Lotfi et al. ${ }^{104}$

Lustig 198

Morsali et al. ${ }^{201}$

May and Mausbach ${ }^{204}$

Yigzawe and Sadus ${ }^{205,206}$

Mairhofer and Sadus ${ }^{207}$

Thol et al. ${ }^{37}$

Köster et al. ${ }^{21}$

Köster et al. ${ }^{21}$

Stephan et al. ${ }^{11}$

Thermal pressure coefficient $\gamma$

McDonald and Singer ${ }^{182}$

Adams ${ }^{189}$

Lustig $^{198}$

Meier 200

Morsali et al. ${ }^{20}$

May and Mausbach ${ }^{204}$

Yigzawe and Sadus ${ }^{205,206}$

Mairhofer and Sadus 207

Thol et al. ${ }^{37}$

Köster et al. ${ }^{21}$

Köster et al. ${ }^{21}$

Stephan et al. ${ }^{11}$

Speed of sound $w$

Meier ${ }^{200}$

Lustig $^{203}$

Yigzawe and Sadus ${ }^{205,206}$

May and Mausbach ${ }^{204}$

Mairhofer and Sadus ${ }^{207}$

$\begin{array}{lll}1967 & 20(9) & 0.72-1.24 \\ 1975 & 12(4) & 2-4 \\ 2012 & 406(48) & 1.3-2.62 \\ 2016 & 197(9) & 0.7-9 \\ 2017 & 45(0) & 1.01-30.0 \\ 2017 & 65(4) & 1.01-30.0 \\ 2019 & 515(11) & 0.7-90\end{array}$

$1967 \quad 22(14) \quad 0.72-1.24$

$197512(9) \quad 2-4$

$197931(21) \quad 1.15-1.35$

$199219(3) \quad 0.7-1.3$

$19942(0) \quad 1.18$

$2007 \quad 13(0) \quad 3.76$

$2012 \quad 205(6) \quad 0.69-6.17$

$2012406(43) \quad 1.3-2.62$

$2013282(6) \quad 1.36-3.05$

$2016197(7) \quad 0.7-9$

$201745(0) \quad 1.01-30.0$

$201765(4) \quad 1.01-30.0$

$2019515(6) \quad 0.7-90$

$1967 \quad 20(19) \quad 0.72-1.24$

1975 12(10) $2-4$

$19942(0) \quad 1.18$

$2002326(14) \quad 0.7-6$

2007 13(0) $\quad 3.76$

$2012 \quad 205(2) \quad 0.69-6.17$

$2012406(20) \quad 1.3-2.62$

$2013282(9) \quad 1.36-3.05$

$2016197(8) \quad 0.7-9$

$201745(0) \quad 1.01-30.0$

$201765(2) \quad 1.01-30.0$

$2019515(5) \quad 0.7-90$

$2002349(20) \quad 0.7-6$

$20118(0) \quad 1.02-3.0$

$2012406(29) \quad 1.3-2.62$

$2012205(8) \quad 0.69-6.17$

$2013 \quad 282(0) \quad 1.36-3.05$

Continued on next page 


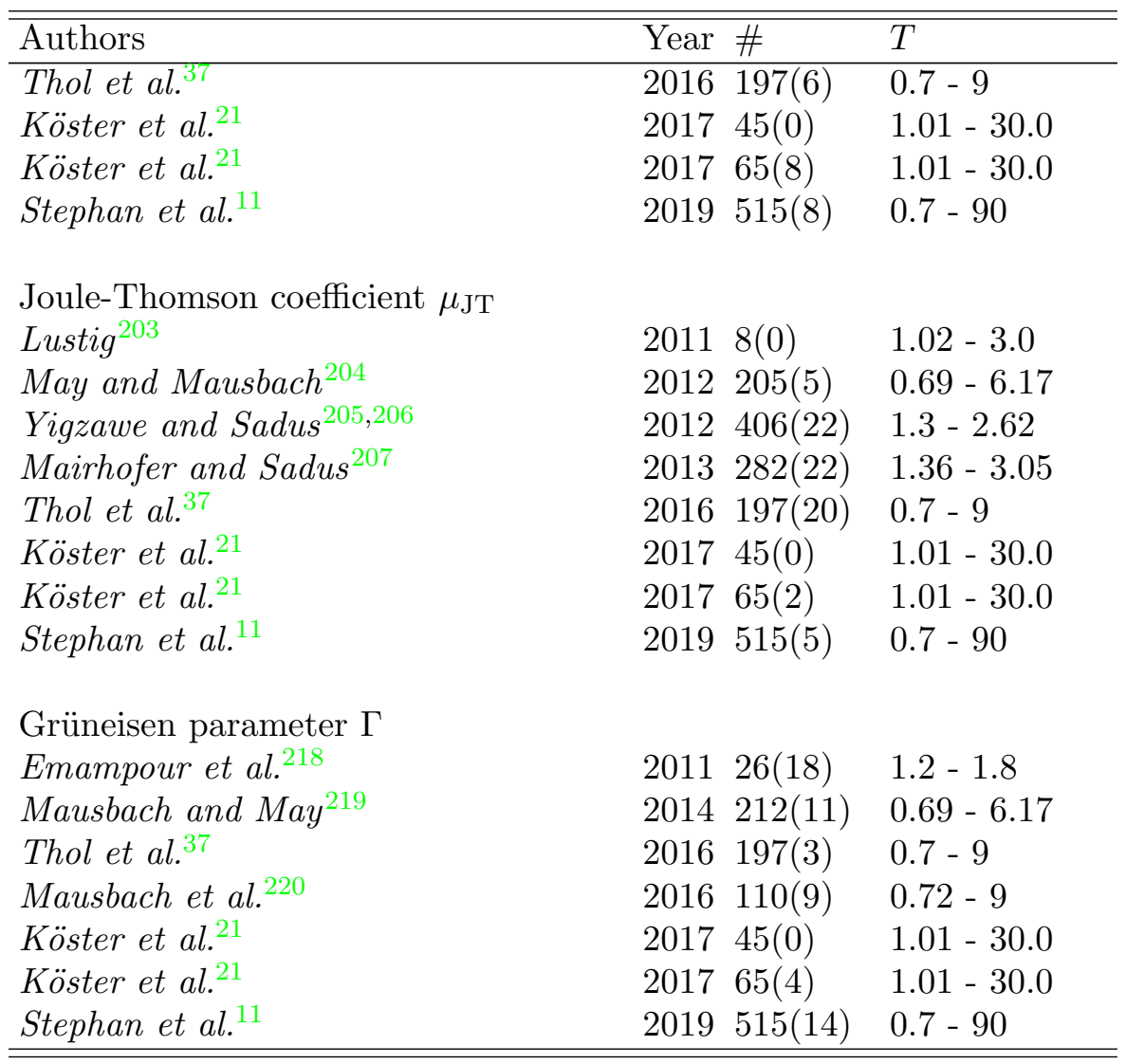

\section{APPENDIX B - PARAMETRIZATION OF NEW LJ EOS BASED ON THE PC-SAFT MONOMER MODEL}

The LJ EOS developed in the present work is a re-parameterization of the PC-SAFT ${ }^{58}$ monomer model, i.e. a spherical particle with soft repulsive and dispersive interactions. The universal constants of the PC-SAFT EOS ${ }^{58}$ have been optimized several times in the literature $^{38,78,166,170,171}$. Gross and Sadowski ${ }^{170}$, who developed the original version of the PC-SAFT EOS adjusted the universal constants to hard chain computer experiment data ${ }^{170}$ and n-alkane VLE properties ${ }^{58}$; Liang and Kontogeorgis ${ }^{166,171}$ fitted the parameters to VLE and speed of sound data of different real substances, and Heier et al. ${ }^{38}$ fitted the parameters to VLE data of the Lennard-Jones truncated and shifted fluid. To include the PC-SAFT monomer model in the comprehensive comparison of the present work, its universal constants were re-parametrized such as to match computer experiment data of the Lennard-Jones fluid.

As most other theoretically-based LJ EOS, the PC-SAFT monomer model is based on perturbation theory ${ }^{86,89,172-174}$. The PC-SAFT monomer model uses the perturbation theory 
of Barker and Henderson ${ }^{86,87}$, according to which the Helmholtz energy of the LJ fluid can be written as

$$
\tilde{a}=\tilde{a}_{\text {id }}+\tilde{a}_{\text {res }}=\tilde{a}_{\text {id }}+\tilde{a}_{\text {ref }}+\tilde{a}_{\text {pert }},
$$

where $\tilde{a}_{\text {ref }}$ and $\tilde{a}_{\text {pert }}$ are the Helmholtz energy contribution of the reference system and the perturbation, respectively.

The Helmholtz energy of the hard sphere fluid of the PC-SAFT monomer is adopted from Boublik ${ }^{91}$ and Mansoori et al. ${ }^{92}$ as

$$
\tilde{a}_{\mathrm{ref}}=\tilde{a}_{\mathrm{hs}}=\zeta_{0}^{-1}\left(\frac{3 \zeta_{1} \zeta_{2}}{\left(1-\zeta_{3}\right)}+\frac{\zeta_{2}^{3}}{\zeta_{3}\left(1-\zeta_{3}\right)^{2}}+\left(\frac{\zeta_{2}^{3}}{\zeta_{3}^{2}}-\zeta_{0}\right) \ln \left(1-\zeta_{3}\right)\right),
$$

where $\zeta_{n}$ is defined as

$$
\zeta_{n}=\frac{\pi}{6} \rho d^{n} \quad \text { with } n=0,1,2,3,
$$

where $\zeta_{3}=\eta$ is the packing fraction and $\rho$ is the number density.

Following Barker and Henderson ${ }^{175}$, the purely repulsively interacting reference fluid is that of a hard sphere, with an effective temperature-dependent hard sphere diameter $d$ as

$$
d(T)=\int_{0}^{1}\left(1-\exp \left(-\frac{u_{\mathrm{LJ}}(r)}{T}\right)\right) \mathrm{d} r .
$$

This temperature-dependent diameter gives the repulsive hard sphere term its softness. The PC-SAFT monomer model simplifies Eq. (23) to an algebraic equation proposed by Chen and Kreglewski $i^{176}$ to avoid the numerical integration to

$$
d(T)=1-c_{1} \cdot \exp \left(-\frac{c_{2}}{T}\right) .
$$

The perturbation contribution $\tilde{a}_{\text {pert }}$ is modeled up to second order in the PC-SAFT monomer model, i.e.

$$
\tilde{a}_{\text {pert }}=\frac{1}{T} \tilde{a}_{1}+\frac{1}{T^{2}} \tilde{a}_{2} .
$$

As described in detail in Refs. ${ }^{87,175}$, applying the local compressibility-version of Barker and Henderson's perturbation theory, the first and second order perturbation terms can be written as

$$
\tilde{a}_{1}=-2 \pi \rho \int_{1}^{\infty} u_{\text {pert }}(r) g_{0}(r) r^{2} \mathrm{~d} r, \quad \text { and }
$$




$$
\tilde{a}_{2}=-\pi \rho\left(1+\frac{8 \eta-2 \eta^{2}}{\left(1-\eta^{4}\right)}\right) \frac{\partial}{\partial \rho}\left[\rho \int_{1}^{\infty} u_{\text {pert }}(r)^{2} g_{0}(r) r^{2} \mathrm{~d} r\right]
$$

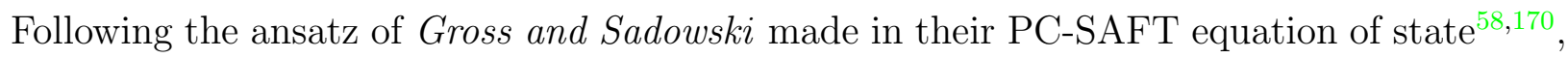
the integrals in Eq. (26) and (27) are simplified into a power series expansion in the packing fraction:

$$
\begin{aligned}
& \int_{1}^{\infty} u_{\text {pert }}(r) g_{0}(r) r^{2} \mathrm{~d} r=I_{1}=\sum_{i=0}^{N} a_{i} \eta^{i} \\
& \frac{\partial}{\partial \rho}\left[\rho \int_{1}^{\infty} u_{\text {pert }}(r)^{2} g_{0}(r) r^{2} \mathrm{~d} r\right]=I_{2}=\sum_{i=0}^{N} b_{i} \eta^{i}
\end{aligned}
$$

The parameters $c_{1}, c_{2}, a_{i}$, and $b_{i}(i=1 \ldots N)$ are the state-independent universal constants of the PC-SAFT monomer model. We chose $N=7$ for the present work. The 18 model parameters were adjusted in the present work to data of the LJ fluid. Only a subset of the computer experiment data compiled in Ref. ${ }^{11}$ was used for that purpose. It comprises consistent and comprehensive data for the VLE and homogeneous fluid states ${ }^{11,37,164}$.

The optimization was guided by the idea that a good representation of VLE properties should be accompanied by a good representation of bulk properties. Therefore, the data set used for the parametrization was a combination of the homogeneous state data and vapor-liquid equilibrium data. Not the full set of simulation data compiled in Ref. ${ }^{11}$ that was used for testing the EOS as described in the main part of the paper was also used for the re-parameterization of the PC-SAFT monomer term. The large and consistent set of simulation data of Thol et al. ${ }^{37}$ for homogeneous stable states was selected here, as it provides the residual Helmholtz energy $\tilde{a}^{\text {res }}=a^{\text {res }} / T$ and its derivatives with respect to the inverse temperature and the density for each state point. The vapor pressure and the saturated densities were taken from the correlations from Ref. ${ }^{11}$ that describe the most precise computer experiment data within their combined scattering. Furthermore, data for the second and third virial coefficient in the range $T=1.32-5$ with a discretization step of 0.005 were used for the parametrization.

The employed parametrization strategy uses elements from multi-criteria optimization to account for conflicts between the different objectives and is based on recent work of our group $^{177-180}$. It is described in detail in the Supplementary Material. 


\section{ACKNOWLEDGMENTS}

The authors gratefully acknowledge funding of the present work by the ERC Advanced Grant ENRICO (grant agreement No. 694807) and by the Deutsche Forschungsgemeinschaft

- DFG (grant IRTG 2057 - 252408385). The present research was conducted under the auspices of the Boltzmann-Zuse Society of Computational Molecular Engineering (BZS). 


\section{REFERENCES}

${ }^{1}$ J. Jones, "On the determination of molecular fields. I. from the variation of the viscosity of a gas with temperature," Proceedings of The Royal Society of London A 106, 441-462 (1924).

${ }^{2}$ J. Jones, "On the determination of molecular fields. II. from the equation of state of a gas," Proceedings of The Royal Society of London A 106, 463-477 (1924).

${ }^{3}$ N. Metropolis, A. W. Rosenbluth, M. N. Rosenbluth, A. H. Teller, and E. Teller, "Equation of state calculations by fast computing machines," The Journal of Chemical Physics 21, 1087-1092 (1953).

${ }^{4}$ M. N. Rosenbluth and A. W. Rosenbluth, "Further results on Monte Carlo equations of state," The Journal of Chemical Physics 22, 881-884 (1954).

${ }^{5} \mathrm{~W}$. W. Wood and F. R. Parker, "Monte Carlo equation of state of molecules interacting with the Lennard-Jones potential. I. A supercritical isotherm at about twice the critical temperature," The Journal of Chemical Physics 27, 720-733 (1957).

${ }^{6}$ B. J. Alder and T. E. Wainwright, "Studies in molecular dynamics. I. general method," The Journal of Chemical Physics 31, 459-466 (1959).

${ }^{7}$ A. Rahman, "Correlations in the motion of atoms in liquid argon," Physical Review 136, A405-A411 (1964).

${ }^{8}$ L. Verlet, "Computer 'experiments' on classical fluids. I. thermodynamical properties of Lennard-Jones molecules," Physical Review 159, 98-103 (1967).

${ }^{9}$ I. R. McDonald and K. Singer, "Machine calculation of thermodynamic properties of a simple fluid at supercritical temperatures," The Journal of Chemical Physics 47, 47664772 (1967).

${ }^{10}$ J.-P. Hansen and L. Verlet, "Phase transitions of the Lennard-Jones system," Physical Review 184, 151-161 (1969).

${ }^{11}$ S. Stephan, M. Thol, J. Vrabec, and H. Hasse, "Thermophysical properties of the Lennard-Jones fluid: Database and data assessment," Journal of Chemical Information and Modeling 59, 4248-4265 (2019).

${ }^{12}$ J. J. Nicolas, K. E. Gubbins, W. B. Streett, and D. J. Tildesley, "Equation of state for the Lennard-Jones fluid," Molecular Physics 37, 1429 (1979).

${ }^{13}$ M. M. Telo da Gama and R. Evans, "The structure and surface tension of the liquid- 
vapour interface near the upper critical end point of a binary mixture of Lennard-Jones fluids I. the two phase region," Molecular Physics 48, 229-250 (1983).

${ }^{14}$ J. D. Honeycutt and H. C. Andersen, "Molecular dynamics study of melting and freezing of small Lennard-Jones clusters," The Journal of Physical Chemistry 91, 4950-4963 (1987).

${ }^{15}$ P. A. Thompson and M. O. Robbins, "Shear flow near solids: Epitaxial order and flow boundary conditions," Physical Review A 41, 6830-6837 (1990).

${ }^{16}$ S. M. Thomson, K. E. Gubbins, J. P. Walton, R. A. Chantry, and J. S. Rowlinson, "A molecular dynamics study of liquid drops," The Journal of Chemical Physics 81, 530 (1984).

${ }^{17}$ P. R. ten Wolde and D. Frenkel, "Computer simulation study of gas-liquid nucleation in a Lennard-Jones system," The Journal of Chemical Physics 109, 9901-9918 (1998).

${ }^{18}$ M. Mecke, J. Winkelmann, and J. Fischer, "Molecular dynamics simulation of the liquidvapor interface: The Lennard-Jones fluid," The Journal of Chemical Physics 107, 92649270 (1997).

${ }^{19}$ A. Mejía, J. C. Pàmies, D. Duque, H. Segura, and L. F. Vega, "Phase and interface behaviors in type-I and type-V Lennard-Jones mixtures: Theory and simulations," The Journal of Chemical Physics 123, 034505 (2005).

${ }^{20} \mathrm{M}$. Horsch, J. Vrabec, and H. Hasse, "Modification of the classical nucleation theory based on molecular simulation data for surface tension, critical nucleus size, and nucleation rate," Physical Review E 78, 011603 (2008).

${ }^{21}$ A. Köster, P. Mausbach, and J. Vrabec, "Premelting, solid-fluid equilibria, and thermodynamic properties in the high density region based on the Lennard-Jones potential," The Journal of Chemical Physics 147, 144502 (2017).

${ }^{22}$ S. Stephan and H. Hasse, "Molecular interactions at vapor-liquid interfaces: Binary mixtures of simple fluids," Physical Review E 101, 012802 (2020).

${ }^{23}$ S. Stephan, J. Liu, K. Langenbach, W. G. Chapman, and H. Hasse, "Vapor-liquid interface of the Lennard-Jones truncated and shifted fluid: Comparison of molecular simulation, density gradient theory, and density functional theory," The Journal of Physical Chemistry C 122, 24705-24715 (2018).

${ }^{24}$ P. Rosales-Pelaez, I. Sanchez-Burgos, C. Valeriani, C. Vega, and E. Sanz, "Seeding approach to nucleation in the NVT ensemble: The case of bubble cavitation in overstretched Lennard Jones fluids," Physical Review E 101, 022611 (2020). 
${ }^{25}$ B. L. Eggimann, A. J. Sunnarborg, H. D. Stern, A. P. Bliss, and J. I. Siepmann, "An online parameter and property database for the TraPPE force field," Molecular Simulation 40, 101-105 (2014).

${ }^{26}$ J. L. F. Abascal and C. Vega, "A general purpose model for the condensed phases of water: TIP4P/2005," The Journal of Chemical Physics 123, 234505 (2005).

${ }^{27}$ S. Stephan, M. Horsch, J. Vrabec, and H. Hasse, "MolMod - an open access database of force fields for molecular simulations of fluids," Molecular Simulation 45, 806-814 (2019).

${ }^{28}$ F. H. Ree, "Analytic representation of thermodynamic data for the Lennard-Jones fluid," The Journal of Chemical Physics 73, 5401-5403 (1980).

${ }^{29}$ R. L. Cotterman, B. J. Schwarz, and J. M. Prausnitz, "Molecular thermodynamics for fluids at low and high densities. Part I: Pure fluids containing small or large molecules," AIChE Journal 32, 1787-1798 (1986).

${ }^{30}$ Y. Adachi, I. Fijihara, M. Takamiya, and K. Nakanishi, "Generalized equation of state for Lennard-Jones fluids - I. pure fluids and simple mixtures," Fluid Phase Equilibria 39, $1-38$ (1988).

${ }^{31}$ U. K. Deiters and K. M. De Reuck, "Guidelines for publication of equations of state - I. pure fluids," Chemical Engineering Journal 69, 69-81 (1998).

${ }^{32}$ R. Span, Multiparameter Equations of State (Springer, Berlin, 2000).

${ }^{33}$ U. K. Deiters and T. Kraska, High-Pressure Fluid Phase Equilibria - Phenomenology and Computation (Elsevier, Amsterdam, 2012).

${ }^{34}$ O. Pohl, "Correspondence - evaluation of an improved volume translation for the prediction of hydrocarbon volumetric properties," Fluid Phase Equilibria 163, 157-159 (1999).

${ }^{35}$ L. V. Yelash and T. Kraska, "Volume-translated equations of state: Empirical approach and physical relevance," AIChE Journal 49, 1569-1579 (2003).

${ }^{36} \mathrm{~J}$. Ahlers and J. Gmehling, "Development of an universal group contribution equation of state: I. prediction of liquid densities for pure compounds with a volume translated Peng-Robinson equation of state," Fluid Phase Equilibria 191, 177-188 (2001).

${ }^{37}$ M. Thol, G. Rutkai, A. Köster, R. Lustig, R. Span, and J. Vrabec, "Equation of state for the Lennard-Jones fluid," Journal of Physical and Chemical Reference Data 45, 023101 (2016).

${ }^{38}$ M. Heier, S. Stephan, J. Liu, W. G. Chapman, H. Hasse, and K. Langenbach, "Equation of state for the Lennard-Jones truncated and shifted fluid with a cut-off radius of 
$2.5 \sigma$ based on perturbation theory and its applications to interfacial thermodynamics," Molecular Physics 116, 2083-2094 (2018).

${ }^{39}$ W. G. Chapman, K. E. Gubbins, G. Jackson, and M. Radosz, "New reference equation of state for associating liquids," Industrial \& Engineering Chemistry Research 29, 1709 (1990).

${ }^{40}$ F. J. Blas and L. F. Vega, "Thermodynamic behaviour of homonuclear and heteronuclear Lennard-Jones chains with association sites from simulation and theory," Molecular Physics 92, 135-150 (1997).

${ }^{41}$ F. J. Blas and L. F. Vega, "Prediction of binary and ternary diagrams using the statistical associating fluid theory (SAFT) equation of state," Industrial \& Engineering Chemistry Research 37, 660-674 (1998).

${ }^{42}$ T. Lafitte, A. Apostolakou, C. Avendano, A. Galindo, C. S. Adjiman, E. A. Müller, and G. Jackson, "Accurate statistical associating fluid theory for chain molecules formed from Mie segments," The Journal of Chemical Physics 139, 154504 (2013).

${ }^{43}$ W. G. Chapman, K. E. Gubbins, G. Jackson, and M. Radosz, "SAFT: Equation-of-state solution model for associating fluids," Fluid Phase Equilibria 52, 31-38 (1989).

${ }^{44}$ K. E. Gubbins, "Perturbation theories of the thermodynamics of polar and associating liquids: A historical perspective," Fluid Phase Equilibria 416, 3-17 (2016).

${ }^{45}$ E. A. Müller and K. E. Gubbins, "Molecular-based equations of state for associating fluids: A review of SAFT and related approaches," Industrial \& Engineering Chemistry Research 40, 2193-2211 (2001).

${ }^{46}$ I. G. Economou, "Statistical associating fluid theory: A successful model for the calculation of thermodynamic and phase equilibrium properties of complex fluid mixtures," Industrial \& Engineering Chemistry Research 41, 953-962 (2002).

${ }^{47}$ Y. Tang and B. C.-Y. Lu, "A study of associating Lennard-Jones chains by a new reference radial distribution function," Fluid Phase Equilibria 171, 27-44 (2000).

${ }^{48}$ E. A. Müeller and K. E. Gubbins, "An equation of state for water from a simplified intermolecular potential," Industrial \& Engineering Chemistry Research 34, 3662-3673 (1995).

${ }^{49}$ T. Kraska and K. E. Gubbins, "Phase equilibria calculations with a modified SAFT equation of state. 1. pure alkanes, alkanols, and water," Industrial \& Engineering Chemistry Research 35, 4727 (1996). 
${ }^{50}$ W. G. Chapman, "Prediction of the thermodynamic properties of associating LennardJones fluids: Theory and simulation," The Journal of Chemical Physics 93, 4299-4304 (1990).

${ }^{51}$ D. Ghonasgi and W. G. Chapman, "Theory and simulation for associating chain fluids," Molecular Physics 80, 161-176 (1993).

${ }^{52}$ D. Ghonasgi and W. G. Chapman, "Prediction of the properties of model polymer solutions and blends," AIChE Journal 40, 878 (1994).

${ }^{53}$ D. Ghonasgi and W. G. Chapman, "Theory and simulation for associating fluids with four bonding sites," Molecular Physics 79, 291-311 (1993).

${ }^{54}$ C.-k. Chen, M. Banaszak, and M. Radosz, "Statistical associating fluid theory equation of state with Lennard-Jones reference applied to pure and binary n-alkane systems," The Journal of Physical Chemistry B 102, 2427-2431 (1998).

${ }^{55}$ V. Papaioannou, T. Lafitte, C. Avendano, C. S. Adjiman, G. Jackson, E. A. Müller, and A. Galindo, "Group contribution methodology based on the statistical associating fluid theory for heteronuclear molecules formed from Mie segments," The Journal of Chemical Physics 140, 054107 (2014).

${ }^{56}$ J. K. Johnson, J. A. Zollweg, and K. E. Gubbins, "The Lennard-Jones equation of state revisited," Molecular Physics 78, 591 (1993).

${ }^{57}$ J. Kolafa and I. Nezbeda, "The Lennard-Jones fluid: an accurate analytic and theoretically-based equation of state," Fluid Phase Equilibria 100, 1-34 (1994).

${ }^{58}$ J. Gross and G. Sadowski, "Perturbed-Chain SAFT: An equation of state based on a perturbation theory for chain molecules," Industrial \& Engineering Chemistry Research 40, 1244 (2001).

${ }^{59}$ J. Gross and G. Sadowski, "Application of the perturbed-chain SAFT equation of state to associating systems," Industrial \& Engineering Chemistry Research 41, 5510-5515 (2002).

${ }^{60}$ M. P. Allen and D. J. Tildesley, Computer Simulation of Liquids (Oxford University Press, Oxford, 1989).

${ }^{61}$ D. Levesque and L. Verlet, "Perturbation theory and equation of state for fluids," Physical Review 182, 307-316 (1969).

${ }^{62}$ S. Toxvaerd and E. Praestgaard, "Equation of state for a Lennard-Jones fluid," The Journal of Chemical Physics 53, 2389-2392 (1970).

${ }^{63}$ I. McDonald and K. Singer, "An equation of state for simple liquids," Molecular Physics 
23, 29-40 (1972).

${ }^{64}$ J. Sýs and A. Malijevský, "Equation of state of a Lennard-Jones 12-6 pairwise additive fluid," Collection of Czechoslovak Chemical Communications 45, 977-983 (1980).

${ }^{65}$ Y. Song and E. A. Mason, "Statistical-mechanical theory of a new analytical equation of state," The Journal of Chemical Physics 91, 7840-7853 (1989).

${ }^{66}$ I. Nezbeda and K. Aim, "On the way from theoretical calculations to practical equations of state for real fluids," Fluid Phase Equilibria 52, 39-46 (1989).

${ }^{67}$ G. M. Sowers and S. I. Sandler, "Equations of state from generalized perturbation theory: Part II. the Lennard-Jones fluid," Fluid Phase Equilibria 67, 127-150 (1991).

${ }^{68}$ N. Koutras, V. Harismiadis, and D. Tassios, "A simple equation of state for the LennardJones fluid: A new reference term for equations of state and perturbation theories," Fluid Phase Equilibria 77, 13-38 (1992).

${ }^{69}$ Y. Miyano, "An equation of state for Lennard-Jones pure fluids applicable over a very wide temperature range," Fluid Phase Equilibria 85, 71-80 (1993).

${ }^{70}$ M. Mecke, A. Müller, J. Winkelmann, J. Vrabec, J. Fischer, R. Span, and W. Wagner, "An accurate van der Waals-type equation of state for the Lennard-Jones fluid," International Journal of Thermophysics 17, 391-404 (1996).

${ }^{71}$ M. Mecke, A. Müller, J. Winkelmann, J. Vrabec, J. Fischer, R. Span, and W. Wagner, "Erratum - an accurate van der Waals-type equation of state for the Lennard-Jones fluid," International Journal of Thermophysics 19, 1493-1498 (1998).

${ }^{72}$ T. Sun and A. S. Teja, "An equation of state for real fluids based on the Lennard-Jones potential," The Journal of Physical Chemistry 100, 17365-17372 (1996).

${ }^{73}$ F. Cuadros, A. Mulero, and W. Ahumada, "An extensive study of the Helmholtz free energy of Lennard-Jones fluids using WCA theory," Thermochimica Acta 277, 85-105 (1996).

${ }^{74}$ A. Amadei, M. E. F. Apol, G. Chillemi, H. J. C. Berendsen, and A. Di Nola, "Derivation of a general fluid equation of state based on the quasi-Gaussian entropy theory: Application to the Lennard-Jones fluid," Molecular Physics 96, 1469-1490 (1999).

${ }^{75}$ S. Hess, "Augmented van der Waals equation of state for the Lennard-Jones fluid," Physica A 267, 58-70 (1999).

${ }^{76}$ G. S. Boltachev and V. G. Baidakov, "Equation of state for Lennard-Jones fluid," High Temperature 41, 270-272 (2003). 
${ }^{77}$ P. Paricaud, "A general perturbation approach for equation of state development: Applications to simple fluids, ab initio potentials, and fullerenes," The Journal of Chemical Physics 124, 154505 (2006).

${ }^{78}$ F. Betancourt-Cardenas, L. Galicia-Luna, and S. Sandler, "Equation of state for the Lennard-Jones fluid based on the perturbation theory," Fluid Phase Equilibria 264, 174183 (2008).

${ }^{79}$ S. E. Quiñones-Cisneros, U. K. Deiters, R. E. Rozas, and T. Kraska, "New model for the correlation of the surface tension based on friction theory," The Journal of Physical Chemistry B 113, 3504-3511 (2009).

${ }^{80}$ H.-O. May and P. Mausbach, "Riemannian geometry study of vapor-liquid phase equilibria and supercritical behavior of the Lennard-Jones fluid," Physical Review E 85, 031201 (2012).

${ }^{81}$ H.-O. May and P. Mausbach, "Erratum: Riemannian geometry study of vapor-liquid phase equilibria and supercritical behavior of the Lennard-Jones fluid [Phys. Rev. E 85, 031201 (2012)]," Physical Review E 86, 059905 (2012).

${ }^{82} \mathrm{~T}$. van Westen and J. Gross, "A critical evaluation of perturbation theories by Monte Carlo simulation of the first four perturbation terms in a Helmholtz energy expansion for the Lennard-Jones fluid," The Journal of Chemical Physics 147, 014503 (2017).

${ }^{83}$ M. Gottschalk, "An EOS for the Lennard-Jones fluid: A virial expansion approach," AIP Advances 9, 125206 (2019).

${ }^{84}$ M. Benedict, G. B. Webb, and L. C. Rubin, "An empirical equation for thermodynamic properties of light hydrocarbons and their mixtures i. methane, ethane, propane and n-butane," The Journal of Chemical Physics 8, 334-345 (1940).

${ }^{85}$ R. T. Jacobsen and R. B. Stewart, "Thermodynamic properties of nitrogen including liquid and vapor phases from $63 \mathrm{~K}$ to $2000 \mathrm{~K}$ with pressures to 10,000 bar," Journal of Physical and Chemical Reference Data 2, 757-922 (1973).

${ }^{86}$ J. A. Barker and D. Henderson, "Theories of liquids," Annual Review of Physical Chemistry 23, 439-484 (1972).

${ }^{87}$ J. A. Barker and D. Henderson, "Perturbation theory and equation of state for fluids: The square-well potential," The Journal of Chemical Physics 47, 2856-2861 (1967).

${ }^{88}$ J. D. Weeks, D. Chandler, and H. C. Andersen, "Perturbation theory of the thermodynamic properties of simple liquids," The Journal of Chemical Physics 55, 5422-5423 
(1971).

${ }^{89}$ J. A. Barker and D. Henderson, "What is "liquid"? understanding the states of matter," Review of modern physics 48, 587-671 (1976).

${ }^{90}$ N. F. Carnahan and K. E. Starling, "Equation of state for nonattracting rigid spheres," The Journal of Chemical Physics 51, 635-636 (1969).

${ }^{91}$ T. Boublík, "Hard-sphere equation of state," The Journal of Chemical Physics 53, 471 (1970).

${ }^{92}$ G. A. Mansoori, N. F. Carnahan, K. E. Starling, and T. W. Leland, "Equilibrium thermodynamic properties of the mixture of hard spheres," The Journal of Chemical Physics 54, 1523 (1971).

${ }^{93} \mathrm{H}$. Hasse and J. Lenhard, "Boon and bane: On the role of adjustable parameters in simulation models," in Mathematics as a Tool: Tracing New Roles of Mathematics in the Sciences, edited by J. Lenhard and M. Carrier (Springer International Publishing, 2017) pp. $93-115$.

${ }^{94}$ M. Schappals, A. Mecklenfeld, L. Kröger, V. Botan, A. Köster, S. Stephan, E. J. Garcia, G. Rutkai, G. Raabe, P. Klein, K. Leonhard, C. W. Glass, J. Lenhard, J. Vrabec, and H. Hasse, "Round robin study: Molecular simulation of thermodynamic properties from models with internal degrees of freedom," Journal of Chemical Theory and Computation 13, 4270-4280 (2017).

${ }^{95}$ J. Powles, W. Evans, and N. Quirke, "Non-destructive molecular-dynamics simulation of the chemical potential of a fluid," Molecular Physics 46, 1347-1370 (1982).

${ }^{96}$ J. K. Lee, J. A. Barker, and G. M. Pound, "Surface structure and surface tension: Perturbation theory and Monte Carlo calculation," The Journal of Chemical Physics 60, 1976-1980 (1974).

${ }^{97}$ D. J. Adams, "Calculating the low temperature vapour line by Monte Carlo," Molecular Physics 32, 647-657 (1976).

${ }^{98}$ G. A. Chapela, G. Saville, S. M. Thompson, and J. S. Rowlinson, "Computer simulation of a gas-liquid surface. part 1," Journal of The Chemical Society, Faraday Transactions 2 73, 1133-1144 (1977).

${ }^{99}$ D. J. Adams, "Calculating the high-temperature vapour line by Monte Carlo," Molecular Physics 37, 211-221 (1979).

${ }^{100}$ A. Z. Panagiotopoulos, "Direct determination of phase coexistence properties of fluids by 
Monte Carlo simulation in a new ensemble," Molecular Physics 61, 813-826 (1987).

${ }^{101}$ A. Z. Panagiotopoulos, N. Quirke, M. Stapleton, and D. J. Tildesley, "Phase equilibria by simulation in the Gibbs ensemble," Molecular Physics 63, 527-545 (1988).

${ }^{102}$ M. J. P. Nijmeijer, A. F. Bakker, C. Bruin, and J. H. Sikkenk, "A molecular dynamics simulation of the Lennard-Jones liquid-vapor interface," The Journal of Chemical Physics 89, 3789-3792 (1988).

${ }^{103}$ B. Smit and D. Frenkel, "Calculation of the chemical potential in the Gibbs ensemble," Molecular Physics 68, 951-958 (1989).

${ }^{104}$ A. Lotfi, J. Vrabec, and J. Fischer, "Vapour liquid equilibria of the Lennard-Jones fluid from the NpT plus test particle method," Molecular Physics 76, 1319-1333 (1992).

${ }^{105}$ D. A. Kofke, "Direct evaluation of phase coexistence by molecular simulation via integration along the saturation line," The Journal of Chemical Physics 98, 4149-4162 (1993).

${ }^{106}$ C. D. Holcomb, P. Clancy, and J. A. Zollweg, "A critical study of the simulation of the liquid-vapour interface of a Lennard-Jones fluid," Molecular Physics 78, 437-459 (1993).

${ }^{107}$ R. Agrawal and D. A. Kofke, "Thermodynamic and structural properties of model systems at solid-fluid coexistence ii. melting and sublimation of the Lennard-Jones system," Molecular Physics 85, 43-59 (1995).

${ }^{108}$ J. E. Hunter and W. P. Reinhardt, "Finite-size scaling behavior of the free energy barrier between coexisting phases: Determination of the critical temperature and interfacial tension of the Lennard-Jones fluid," The Journal of Chemical Physics 103, 8627-8637 (1995).

${ }^{109}$ R. J. Sadus and J. M. Prausnitz, "Three-body interactions in fluids from molecular simulation: Vapor-liquid phase coexistence of argon," The Journal of Chemical Physics 104, 4784-4787 (1996).

${ }^{110}$ D. Plačkov and R. J. Sadus, "Molecular simulation of intermolecular attraction and repulsion in coexisting liquid and vapour phases," Fluid Phase Equilibria 134, 77-85 (1997).

${ }^{111}$ M. Guo, D.-Y. Peng, and B. C.-Y.Lu, "On the long-range corrections to computer simulation results for the Lennard-Jones vapor-liquid interface," Fluid Phase Equilibria 130, 19-30 (1997).

${ }^{112}$ M. Guo and B. C.-Y. Lu, "Long range corrections to thermodynamic properties of inhomogeneous systems with planar interfaces," The Journal of Chemical Physics 106, 3688-3695 (1997). 
${ }^{113}$ M. G. Martin and J. I. Siepmann, "Transferable potentials for phase equilibria. 1. Unitedatom description of n-alkanes," The Journal of Physical Chemistry B 102, 2569-2577 (1998).

${ }^{114}$ A. Trokhymchuk and J. Alejandre, "Computer simulations of liquid/vapor interface in Lennard-Jones fluids: Some questions and answers," The Journal of Chemical Physics 111, 8510-8523 (1999).

${ }^{115}$ S. I. Anisimov, D. O. Dunikov, V. V. Zhakhovskii, and S. P. Malyshenko, "Properties of a liquid-gas interface at high-rate evaporation," The Journal of Chemical Physics 110, 8722-8729 (1999).

${ }^{116}$ J. J. Potoff and A. Z. Panagiotopoulos, "Surface tension of the three-dimensional LennardJones fluid from histogram-reweighting Monte Carlo simulations," The Journal of Chemical Physics 112, 6411-6415 (2000).

${ }^{117}$ V. Baidakov, G. Chernykh, and S. Protsenko, "Effect of the cut-off radius of the intermolecular potential on phase equilibrium and surface tension in Lennard-Jones systems," Chemical Physics Letters 321, 315-320 (2000).

${ }^{118}$ H. Okumura and F. Yonezawa, "Liquid-vapor coexistence curves of several interatomic model potentials," The Journal of Chemical Physics 113, 9162-9168 (2000).

${ }^{119}$ W. Shi and J. Johnson, "Histogram reweighting and finite-size scaling study of the Lennard-Jones fluids," Fluid Phase Equilibria 187, 171-191 (2001).

${ }^{120}$ B. Chen, J. I. Siepmann, and M. L. Klein, "Direct Gibbs Ensemble Monte Carlo simulations for solid-vapor phase equilibria: Applications to Lennard-Jonesium and carbon dioxide," The Journal of Physical Chemistry B 105, 9840-9848 (2001).

${ }^{121}$ H. Okumura and F. Yonezawa, "Reliable determination of the liquid-vapor critical point by the NVT plus test particle method," Journal of The Physical Society of Japan 70, 1990-1994 (2001).

${ }^{122}$ V. G. Baidakov, S. P. Protsenko, G. G. Chernykh, and G. S. Boltachev, "Statistical substantiation of the van der Waals theory of inhomogeneous fluids," Physical Review E 65, 041601 (2002).

${ }^{123}$ L. I. Kioupis, G. Arya, and E. J. Maginn, "Pressure-enthalpy driven molecular dynamics for thermodynamic property calculation II: Applications," Fluid Phase Equilibria 200, 93-110 (2002).

${ }^{124}$ J. R. Errington, "Evaluating surface tension using grand-canonical transition-matrix 
Monte Carlo simulation and finite-size scaling," Physical Review E 67, 012102 (2003).

${ }^{125}$ J. R. Errington, "Direct calculation of liquid-vapor phase equilibria from transition matrix Monte Carlo simulation," The Journal of Chemical Physics 118, 9915-9925 (2003).

${ }^{126}$ J. Stoll, J. Vrabec, and H. Hasse, "Comprehensive study of the vapour-liquid equilibria of the pure two-centre Lennard-Jones plus pointdipole fluid," Fluid Phase Equilibria 209, 29-53 (2003).

${ }^{127}$ V. G. Baidakov, S. P. Protsenko, Z. R. Kozlova, and G. G. Chernykh, "Metastable extension of the liquid-vapor phase equilibrium curve and surface tension," The Journal of Chemical Physics 126, 214505 (2007).

${ }^{128}$ J. Janeček, "Effect of the interfacial area on the equilibrium properties of Lennard-Jones fluid," The Journal of Chemical Physics 131, 124513 (2009).

${ }^{129}$ G. Galliero, M. M. Piñeiro, B. Mendiboure, C. Miqueu, T. Lafitte, and D. Bessieres, "Interfacial properties of the Mie n-6 fluid: Molecular simulations and gradient theory results," The Journal of Chemical Physics 130, 104704 (2009).

${ }^{130}$ R. J. Sadus, "Molecular simulation of the phase behavior of fluids and fluid mixtures using the synthetic method," The Journal of Chemical Physics 137, 054507 (2012).

${ }^{131}$ J. Mick, E. Hailat, V. Russo, K. Rushaidat, L. Schwiebert, and J. Potoff, "GPUaccelerated Gibbs ensemble Monte Carlo simulations of Lennard-Jonesium," Computer Physics Communications 184, 2662-2669 (2013).

${ }^{132}$ F. J. Martinez-Ruiz, F. J. Blas, B. Mendiboure, and A. I. Moreno-Ventas Bravo, "Effect of dispersive long-range corrections to the pressure tensor: The vapour-liquid interfacial properties of the Lennard-Jones system revisited," The Journal of Chemical Physics 141, 184701 (2014).

${ }^{133}$ J. Janeček, O. Said-Aizpuru, and P. Paricaud, "Long range corrections for inhomogeneous simulations of Mie n-m potential," Journal of Chemical Theory and Computation 13, 4482-4491 (2017).

${ }^{134}$ S. Werth, K. Stöbener, M. Horsch, and H. Hasse, "Simultaneous description of bulk and interfacial properties of fluids by the Mie potential," Molecular Physics 115, 1017-1030 (2017).

${ }^{135}$ S. Stephan and H. Hasse, "Influence of dispersive long-range interactions on properties of vapour-liquid equilibria and interfaces of binary Lennard-Jones mixtures," Molecular Physics 118, e1699185 (2020). 
${ }^{136}$ J. V. Sengers and J. M. H. L. Sengers, "Thermodynamic behavior of fluids near the critical point," Annual Review of Physical Chemistry 37, 189-222 (1986).

${ }^{137}$ S. B. Kiselev and J. F. Ely, "Crossover SAFT equation of state: Application for normal alkanes," Industrial \& Engineering Chemistry Research 38, 4993-5004 (1999).

${ }^{138}$ M. E. Fisher, "Renormalization group theory: Its basis and formulation in statistical physics," Reviews of Modern Physics 70, 653-681 (1998).

${ }^{139}$ J. A. Barker, P. J. Leonard, and A. Pompe, "Fifth virial coefficients," The Journal of Chemical Physics 44, 4206-4211 (1966).

${ }^{140}$ J. M. Caillol, "Critical-point of the Lennard-Jones fluid: A finite-size scaling study," The Journal of Chemical Physics 109, 4885-4893 (1998).

${ }^{141}$ D. O. Dunikov, S. P. Malyshenko, and V. V. Zhakhovskii, "Corresponding states law and molecular dynamics simulations of the Lennard-Jones fluid," The Journal of Chemical Physics 115, 6623-6631 (2001).

${ }^{142}$ S. Kim, D. Henderson, and J. A. Barker, "Perturbation theory of fluids and deviations from classical behavior," Canadian Journal of Physics 47, 99-102 (1969).

${ }^{143}$ D. A. Kofke, "Gibbs-Duhem integration: A new method for direct evaluation of phase coexistence by molecular simulation," Molecular Physics 78, 1331-1336 (1993).

${ }^{144}$ J. J. Potoff and A. Z. Panagiotopoulos, "Critical point and phase behavior of the pure fluid and a Lennard-Jones mixture," The Journal of Chemical Physics 109, 10914-10920 (1998).

${ }^{145}$ B. Smit, "Phase diagrams of Lennard-Jones fluids," The Journal of Chemical Physics 96, 8639-8640 (1992).

${ }^{146}$ S. H. Sung and D. Chandler, "Optimized cluster theory, the Lennard-Jones fluid, and the liquid-gas phase transition," Physical Review A 9, 1688-1697 (1974).

${ }^{147}$ L. Verlet and D. Levesque, "On the theory of classical fluids VI," Physica 36, 254-268 (1967).

${ }^{148}$ V. L. Kulinskii, "The critical compressibility factor of fluids from the global isomorphism approach," The Journal of Chemical Physics 139, 184119 (2013).

${ }^{149}$ D. M. Heyes and L. V. Woodcock, "Critical and supercritical properties of Lennard-Jones fluids," Fluid Phase Equilibria 356, 301-308 (2013).

${ }^{150}$ D. M. Heyes, "The Lennard-Jones fluid in the liquid-vapour critical region," Computational Methods in Science and Technology 21, 169 (2015). 
${ }^{151}$ S. V. Lishchuk and J. Fischer, "Communication: Long range corrections in liquid-vapor interface simulations," The Journal of Chemical Physics 149, 091102 (2018).

${ }^{152}$ A. Meroni, A. Parola, and L. Reatto, "Differential approach to the theory of fluids," Physical Review A 42, 6104-6115 (1990).

${ }^{153}$ N. M. Alsaifi, "Simulation-based equations of state for the Lennard-Jones fluid: Apparent success and hidden failure," AIChE Journal 66, e16244 (2020).

${ }^{154}$ R. Evans, Fundamentals of Inhomogeneous Fluids, edited by D. Henderson (Marcel Dekker, New York, 1992).

${ }^{155}$ R. Evans, "The nature of the liquid-vapour interface and other topics in the statistical mechanics of non-uniform, classical fluids," Advances in Physics 28, 143-200 (1979).

${ }^{156}$ J. S. Rowlinson and B. Widom, Molecular Theory of Capillarity (Dover Publications, New York, 1982).

${ }^{157}$ S. Stephan, K. Langenbach, and H. Hasse, "Interfacial properties of binary LennardJones mixtures by molecular simulations and density gradient theory," The Journal of Chemical Physics 150, 174704 (2019).

${ }^{158}$ S. Stephan, S. Becker, K. Langenbach, and H. Hasse, "Vapor-liquid interfacial properties of the binary system cyclohexane + CO2: Experiment, molecular simulation and density gradient theory," Fluid Phase Equilibria 518, 112583 (2020).

${ }^{159}$ A. Linhart, C.-C. Chen, J. Vrabec, and H. Hasse, "Thermal properties of the metastable supersaturated vapor of the Lennard-Jones fluid," The Journal of Chemical Physics 122, 144506 (2005).

${ }^{160}$ R. J. Wheatley, "Calculation of high-order virial coefficients with applications to hard and soft spheres," Physical Review Letters 110, 200601 (2013).

${ }^{161}$ J. O. Hirschfelder, C. F. Curtiss, and R. B. Bird, Molecular Theory of Gases and Liquids (Wiley, New York, 1954).

${ }^{162}$ K. R. S. Shaul, A. J. Schultz, and D. A. Kofke, "The effect of truncation and shift on virial coefficients of Lennard-Jones potentials," Collection of Czechoslovak Chemical Communications 75, 447-462 (2010).

${ }^{163}$ R. B. Bird, E. L. Spotz, and J. O. Hirschfelder, "The third virial coefficient for non-polar gases," The Journal of Chemical Physics 18, 1395-1402 (1950).

${ }^{164}$ S. Stephan and U. K. Deiters, "Characteristic curves of the Lennard-Jones fluid," submitted (2020). 
${ }^{165}$ O. L. Boshkova and U. K. Deiters, "Soft repulsion and the behavior of equations of state at high pressures," International Journal of Thermophysics 31, 227-252 (2010).

${ }^{166}$ X. Liang, B. Maribo-Mogensen, K. Thomsen, W. Yan, and G. M. Kontogeorgis, "Approach to improve speed of sound calculation within PC-SAFT framework," Industrial \& Engineering Chemistry Research 51, 14903-14914 (2012).

${ }^{167}$ N. I. Diamantonis and I. G. Economou, "Evaluation of statistical associating fluid theory (SAFT) and Perturbed Chain-SAFT equations of state for the calculation of thermodynamic derivative properties of fluids related to carbon capture and sequestration," Energy \& Fuels 25, 3334-3343 (2011).

${ }^{168}$ I. Polishuk, "Implementation of SAFT+cubic and PC-SAFT for comprehensive description of thermodynamic properties of n-octane and its mixtures," The Journal of Supercritical Fluids 62, 47-54 (2012).

${ }^{169}$ I. Polishuk, "Implementation of SAFT + cubic, PC-SAFT, and Soave-Benedict-WebbRubin equations of state for comprehensive description of thermodynamic properties in binary and ternary mixtures of $\mathrm{ch}_{4}, \mathrm{co}_{2}$, and $\mathrm{n}-\mathrm{c}_{16} \mathrm{~h}_{34}$," Industrial \& Engineering Chemistry Research 50, 14175-14185 (2011).

${ }^{170}$ J. Gross and G. Sadowski, "Application of perturbation theory to a hard-chain reference fluid: an equation of state for square-well chains," Fluid Phase Equilibria 168, 183 (2000).

${ }^{171} \mathrm{X}$. Liang and G. M. Kontogeorgis, "New variant of the universal constants in the perturbed chain-statistical associating fluid theory equation of state," Industrial \& Engineering Chemistry Research 54, 1373-1384 (2015).

172 J.-P. Hansen and I. McDonald, Theory of Simple Liquids, 4th ed. (Academic Press, 2013). ${ }^{173}$ W. R. Smith, D. Henderson, and J. A. Barker, "Approximate evaluation of the secondorder term in the perturbation theory of fluids," The Journal of Chemical Physics 53, 508-515 (1970).

${ }^{174}$ D. Henderson and J. Barker, Physical Chemistry - An Advanced Treatise: Volume VIIIA / Liquid State, edited by D. Henderson (Academic Press, New York, 1971).

${ }^{175}$ J. A. Barker and D. Henderson, "Perturbation theory and equation of state for fluids. II. a successful theory of liquids," The Journal of Chemical Physics 47, 4714 (1967).

${ }^{176}$ S. S. Chen and A. Kreglewski, "Applications of the augmented van der Waals theory of fluids.: I. pure fluids," Berichte der Bunsengesellschaft für physikalische Chemie 81, 1048 (1977). 
${ }^{177}$ M. Bortz, V. Maag, J. Schwientek, R. Benfer, R. Böttcher, J. Burger, E. von Harbou, N. Asprion, K.-H. Küfer, and H. Hasse, "Decision support by multicriteria optimization in process development: An integrated approach for robust planning and design of plant experiments," in 12th International Symposium on Process Systems Engineering and 25th European Symposium on Computer Aided Process Engineering, Computer Aided Chemical Engineering, Vol. 37, edited by K. V. Gernaey, J. K. Huusom, and R. Gani (2015) pp. 2063-2068.

${ }^{178}$ M. Bortz, J. Burger, N. Asprion, S. Blagov, R. Böttcher, U. Nowak, A. Scheithauer, R. Welke, K.-H. Küfer, and H. Hasse, "Multi-criteria optimization in chemical process design and decision support by navigation on Pareto sets," Computers \& Chemical Engineering 60, 354-363 (2014).

${ }^{179}$ K. Stöbener, P. Klein, M. Horsch, K. Küfer, and H. Hasse, "Parametrization of two-center Lennard-Jones plus point-quadrupole force field models by multicriteria optimization," Fluid Phase Equilibria 411, 33-42 (2016).

${ }^{180}$ E. Forte, J. Burger, K. Langenbach, H. Hasse, and M. Bortz, "Multi-criteria optimization for parameterization of SAFT-type equations of state for water," AIChE Journal 64, 226237 (2018).

${ }^{181}$ W. Fickett and W. W. Wood, "Shock Hugoniots for liquid argon," Physics of Fluids 3, 204-209 (1960).

${ }^{182}$ I. R. McDonald and K. Singer, "Calculation of thermodynamic properties of liquid argon from Lennard-Jones parameters by a Monte Carlo method," Discussions of The Faraday Society 43, 40-49 (1967).

${ }^{183}$ W. W. Wood, Physics of Simple Liquids, edited by H. N. V. Temperley, J. S. Rowlinson, and G. S. Rushbrooke (North Holland Publishing Company, Amsterdam, 1968) pp. 115230.

${ }^{184}$ I. R. McDonald and K. Singer, "Examination of the adequacy of the 12-6 potential for liquid argon by means of Monte Carlo calculations," The Journal of Chemical Physics 50, 2308-2315 (1969).

${ }^{185}$ J.-P. Hansen, "Phase transition of the Lennard-Jones system. II. high-temperature limit," Physical Review A 2, 221-230 (1970).

${ }^{186}$ I. R. McDonald and L. V. Woodcock, "Triple-dipole dispersion forces in dense fluids," Journal of Physics C 3, 722 (1970). 
${ }^{187}$ P. Schofield, "Computer simulation studies of the liquid state," Computer Physics Communications 5, 17-23 (1973).

${ }^{188}$ W. B. Streett, H. J. Raveché, and R. D. Mountain, "Monte Carlo studies of the fluidsolid phase transition in the Lennard-Jones system," The Journal of Chemical Physics 61, 1960-1969 (1974).

${ }^{189}$ D. J. Adams, "Grand canonical ensemble Monte Carlo for a Lennard-Jones fluid," Molecular Physics 29, 307-311 (1975).

${ }^{190}$ D. D. Carley, "Integral equation and perturbation method for equations of state for a low temperature Lennard-Jones gas," The Journal of Chemical Physics 67, 4812-4818 (1977).

${ }^{191}$ J. Yao, R. Greenkorn, and K. Chao, "Monte Carlo simulation of the grand canonical ensemble," Molecular Physics 46, 587-594 (1982).

${ }^{192}$ K. Lucas, Angewandte Statistische Thermodynamik, 1st ed. (Springer Verlag, Berlin, 1986).

${ }^{193}$ M. S. Shaw, "A density of states transformation Monte Carlo method: Thermodynamics of the Lennard-Jones fluid," The Journal of Chemical Physics 89, 2312-2323 (1988).

${ }^{194}$ A. Baranyai and D. J. Evans, "Direct entropy calculation from computer simulation of liquids," Physical Review A 40, 3817-3822 (1989).

${ }^{195}$ B. Saager and J. Fischer, "Predictive power of effective intermolecular pair potentials: MD simulation results for methane up to 1000 MPa," Fluid Phase Equilibria 57, 35-46 (1990).

${ }^{196}$ P. V. Giaquinta, G. Giunta, and S. Prestipino Giarritta, "Entropy and the freezing of simple liquids," Physical Review A 45, R6966-R6968 (1992).

${ }^{197}$ J. Kolafa, H. L. Vörtler, K. Aim, and I. Nezbeda, "The Lennard-Jones fluid revisited: Computer simulation results," Molecular Simulation 11, 305-319 (1993).

${ }^{198}$ R. Lustig, "Statistical thermodynamics in the classical molecular dynamics ensemble. III. numerical results," The Journal of Chemical Physics 100, 3068-3078 (1994).

${ }^{199}$ D. Roccatano, A. Amadei, M. E. F. Apol, A. Di Nola, and H. J. C. Berendsen, "Application of the quasi-Gaussian entropy theory to molecular dynamics simulations of LennardJones fluids," The Journal of Chemical Physics 109, 6358-6363 (1998).

${ }^{200} \mathrm{~K}$. Meier, Computer Simulation and Interpretation of the Transport Coefficients of the Lennard-Jones Model Fluid, Dissertation, University of the Federal Armed Forces Hamburg (2002). 
${ }^{201}$ A. Morsali, S. A. Beyramabadi, and M. R. Bozorgmehr, "Evaluation of P-V-T differential properties of the Lennard-Jones fluid using radial distribution functions and molecular dynamics," Chemical Physics 335, 194-200 (2007).

${ }^{202}$ V. G. Baidakov, S. P. Protsenko, and Z. R. Kozlova, "Thermal and caloric equations of state for stable and metastable Lennard-Jones fluids: I. molecular-dynamics simulations," Fluid Phase Equilibria 263, 55-63 (2008).

${ }^{203}$ R. Lustig, "Direct molecular NVT simulation of the isobaric heat capacity, speed of sound and Joule-Thomson coefficient," Molecular Simulation 37, 457-465 (2011).

${ }^{204}$ H.-O. May and P. Mausbach, "Fluid properties from equations of state compared with direct molecular simulations for the Lennard-Jones system," AIP Conference Proceedings 1501, 954-960 (2012).

${ }^{205}$ T. M. Yigzawe, Molecular Dynamics Simulation of the Thermodynamic Properties of Water and Atomistic Fluids, Dissertation, Swinburne University of Technology, Melbourne (2012).

${ }^{206}$ T. M. Yigzawe and R. J. Sadus, "Intermolecular interactions and the thermodynamic properties of supercritical fluids," The Journal of Chemical Physics 138, 194502 (2013).

${ }^{207}$ J. Mairhofer and R. J. Sadus, "Thermodynamic properties of supercritical n-m LennardJones fluids and isochoric and isobaric heat capacity maxima and minima," The Journal of Chemical Physics 139, 154503 (2013).

${ }^{208}$ U. K. Deiters and A. Neumaier, "Computer simulation of the characteristic curves of pure fluids," Journal of Chemical \& Engineering Data 61, 2720-2728 (2016).

${ }^{209}$ E. A. Ustinov, "Efficient chemical potential evaluation with kinetic Monte Carlo method and non-uniform external potential: Lennard-Jones fluid, liquid, and solid," The Journal of Chemical Physics 147, 014105 (2017).

${ }^{210}$ A. J. Schultz and D. A. Kofke, "Comprehensive high-precision high-accuracy equation of state and coexistence properties for classical Lennard-Jones crystals and low-temperature fluid phases," The Journal of Chemical Physics 149, 204508 (2018).

${ }^{211}$ J. D. Weeks, D. Chandler, and H. C. Andersen, "Role of repulsive forces in determining the equilibrium structure of simple liquids," The Journal of Chemical Physics 54, 52375247 (1971).

${ }^{212}$ G. Torrie and J. Valleau, "Nonphysical sampling distributions in Monte Carlo free-energy estimation: Umbrella sampling," Journal of Computational Physics 23, 187-199 (1977). 
${ }^{213}$ B. Saager, A. Lotfi, M. Bohn, V. N. Nguyen, and J. Fischer, "Prediction of gas PVT data with effective intermolecular potentials using the Haar-Shenker-Kohler equation and computer simulations," Fluid Phase Equilibria 54, 237 - 246 (1990).

${ }^{214}$ D. Boda, T. Lukács, J. Liszi, and I. Szalai, "The isochoric-, isobaric- and saturation-heat capacities of the Lennard-Jones fluid from equations of state and Monte Carlo simulations," Fluid Phase Equilibria 119, 1-16 (1996).

${ }^{215}$ S. D. Hong and M. S. Jhon, "Calculation of excess free energy from the averaged effective acceptance ratio for the Lennard-Jones fluid and the inverse twelve fluid," Chemical Physics Letters 273, 79-82 (1997).

${ }^{216}$ S.-D. Hong and D.-J. Jang, "Direct calculation of the free energy using reduced Boltzmann factors: Application to Lennard-Jones fluids and square-well fluids," Bulletin of The Korean Chemical Society 24, 1351-1353 (2003).

${ }^{217}$ K.-K. Han, "A new Monte Carlo method for estimating free energy and chemical potential," Physics Letters A 165, 28-32 (1992).

${ }^{218}$ J. S. Emampour, A. Morsali, S. A. Beyramabadi, M. R. Bozorgmehr, and K. Khakzadan, "The pseudo Grüneisen parameter in dense fluids from distribution functions," International Journal of The Physical Sciences 6, 5731-5737 (2011).

${ }^{219}$ P. Mausbach and H.-O. May, "Direct molecular simulation of the Grüneisen parameter and density scaling exponent in fluid systems," Fluid Phase Equilibria 366, 108-116 (2014).

${ }^{220}$ P. Mausbach, A. Köster, G. Rutkai, M. Thol, and J. Vrabec, "Comparative study of the Grüneisen parameter for 28 pure fluids," The Journal of Chemical Physics 144, 244505 (2016). 